DOE/ER/13855--4

DE92 005303

\title{
Capacitance of Edge Plane of Pyrolytic Graphite in Acetonitrile Solutions
}

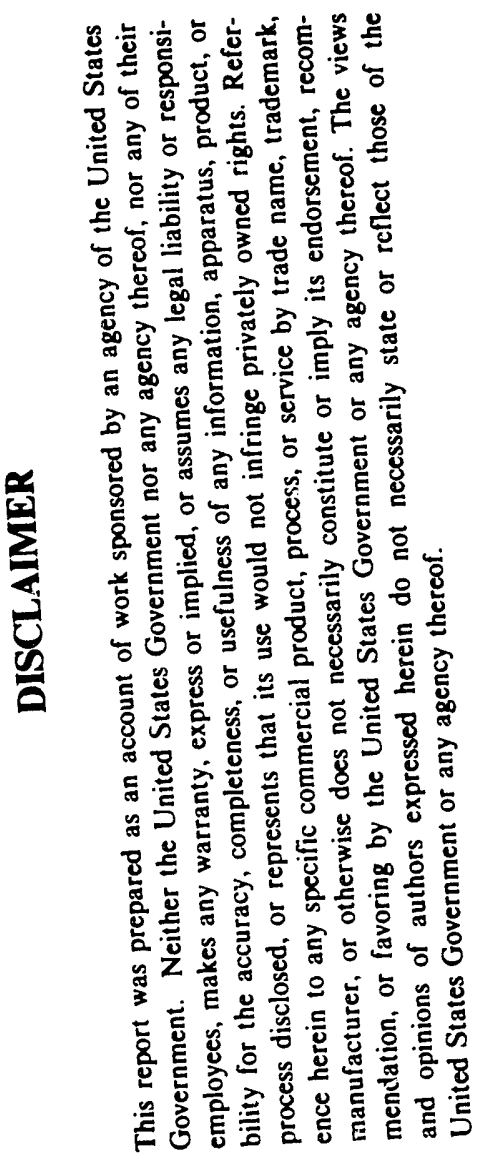

Steven Kent Minick and Takanobu Ishida

\author{
Department of Chemistry \\ State University of New York \\ at \\ Stony Brook
}

Stony Brook, New York 11794

May 1991

Prepared for

THE U.S. DEPARTMENT OF ENERGY

OFFICE OF BASIC ENERGY SCIENCES

Grant No. DE-FG02-88ER13855

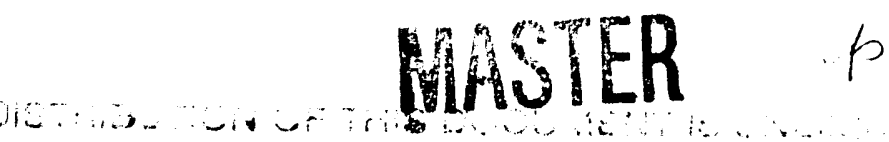




\title{
Cr pacitance of Edge Plane of Pyrolytic Graphite in Acetonitrile Solutions
}

\author{
A Thesis Presented \\ by \\ Steven Kent Minick
}

to

\author{
The Graduate School \\ in Partial Fulfillment of the Requirements \\ for the Degree of \\ Master of Science \\ in \\ Chenistry \\ State University of New York \\ at Stony Brook
}

May, 1991 


\section{State University of New York \\ at Stony Brook \\ The Graduate School}

\section{Steven Minick}

We, the thesis committee for the above candidate for the Master of Science degree, hereby recommend acceptance of this thesis.

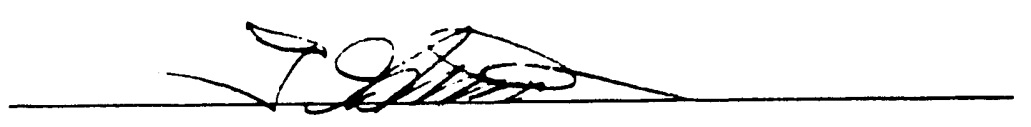

Professor Takanobu Ishida, Advisor

Department of Chemistry

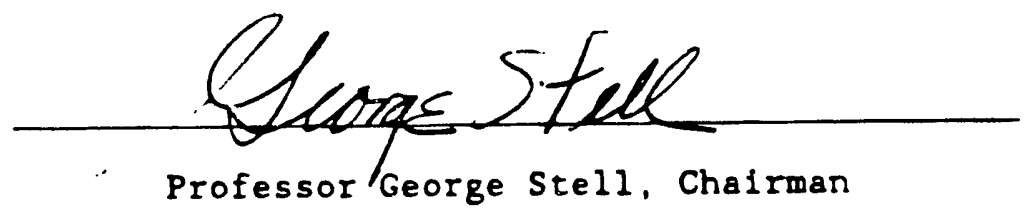

Department of Chemistry

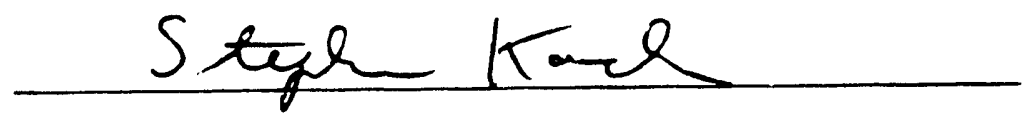

Professor Stephen A. Koch

Department of Chemistry

This thesis is accepted by the Graduate school.

The Graduate School 
Abstract of the Thesis

\title{
Capacitance of Bdge Plane of Pyrolytic Graphite in \\ Acetonitrile Solutions.
}

by

\section{Steven Kent Minick}

\author{
Master of Science \\ in \\ Chemistry
}

State University of New York at Stony Brook

1991

The capacitance of the edge plane of pyrolytic graphite electrodes, in acetonitrile solutions, is measured by recording the current response to an applied triangular voltage sweep; TVS, and then fitting the current response with an appropriate function, (via a set of adjustable parameters). The pretreatment 
of the electrodes, the supporting electrolyte concentration used, and the frequency of the input TVS, were all found to affect the measured capacitance. In these experiments, a background current was also seen and the shape of the current output for the TVS; the charging/discharging curve, is shown to correlate with the magnitule of this background current. In addition, the size of the background current was found to have some dependence on the type of electrode pretreatment procedure used. 
Table of Contents

List of Figures viii

List of Tables $x$

Acknowledgments

$x i$

I. Introduction 1

Purpose of this study $\ldots \ldots \ldots \ldots \ldots \ldots \ldots \ldots \ldots$

Electric Double Layer Structure $\ldots \ldots \ldots \ldots \ldots \ldots \ldots 2$

Ideal Polarized Electrode $\ldots \ldots \ldots \ldots \ldots \ldots \ldots \ldots$

Electrocapillarity $\quad \ldots \ldots \ldots \ldots \ldots \ldots \ldots \ldots \ldots$

Integral vs Differential Capacitance $\ldots \ldots \ldots \ldots \ldots \ldots$

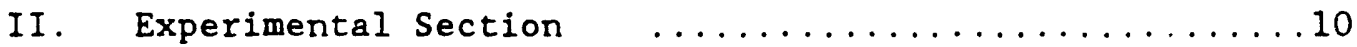

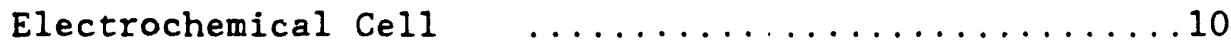

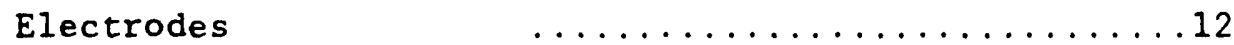

Chemicals and Materials used $\ldots \ldots \ldots \ldots \ldots \ldots \ldots 17$

Instrumentation, Hardware and Software $\ldots \ldots \ldots \ldots \ldots$

Electrode Pretreatment Procedures ............... 19

Optional Pretreatment Procedures .............21

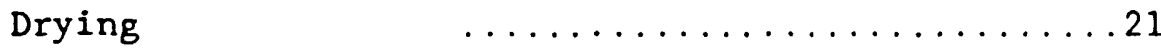

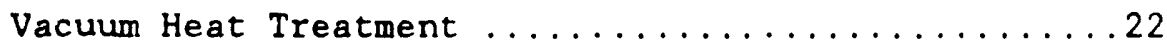

Radio Frequency Plasma Treatment ...........26

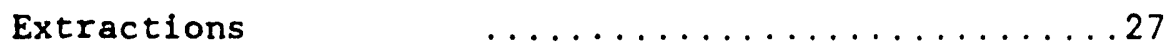

III. Capacitance Measurements; Experimental Problems

and Questions of Technique .................... 
Which Measurement Technique to use $\ldots \ldots \ldots \ldots \ldots 28$

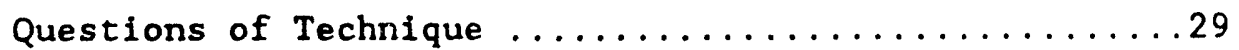

Noise in the Current Response ................. 31

Destabilization of the Potential ................. 35

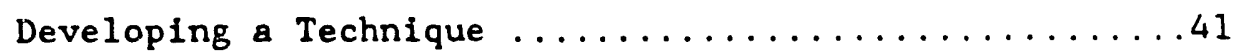

Measuring the Capacitance $\ldots \ldots \ldots \ldots \ldots \ldots \ldots \ldots \ldots$

Results from this work $\ldots \ldots \ldots \ldots \ldots \ldots \ldots \ldots$

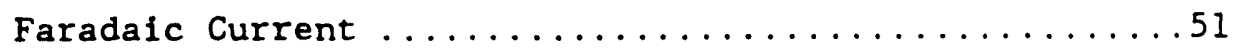

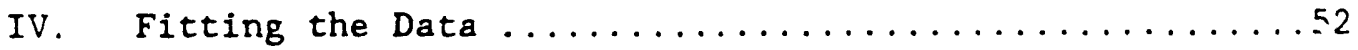

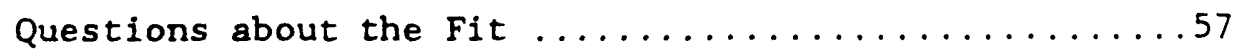

Fitting including a faradaic Term $\ldots \ldots \ldots \ldots \ldots \ldots 2$

Assume no faradaic Current ...............64

v. Results and Discussion ..................64

Capacitance vs Potential Curves ..............64

Reproducible C-E Curves .................66

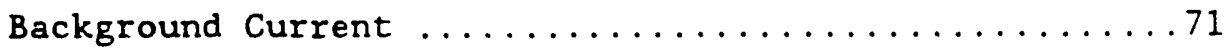

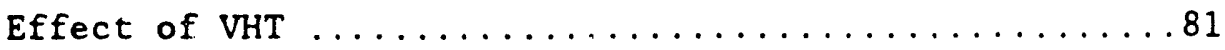

Effect of Supporting Electrolyte Concentration ......83

Effect on the Resistances $\ldots \ldots \ldots \ldots \ldots \ldots \ldots \ldots$

Frequency Dependence of the Capacitances ........92

VI. Conclusions $\ldots \ldots \ldots \ldots \ldots \ldots \ldots \ldots \ldots \ldots \ldots \ldots \ldots \ldots \ldots \ldots \ldots$

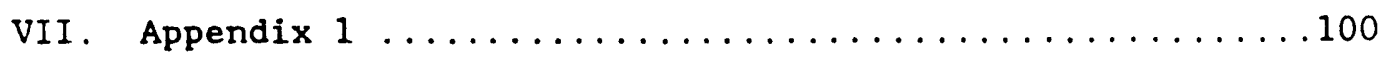

Cyclic Voltammetry of the Edge Plane of P.G.

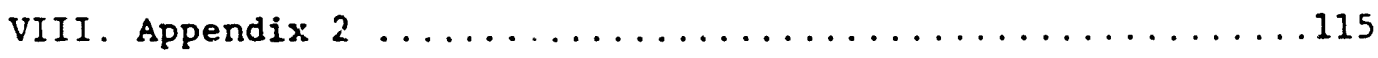

Working Ranges: limits to the sizes of acceptable applied 
potentials

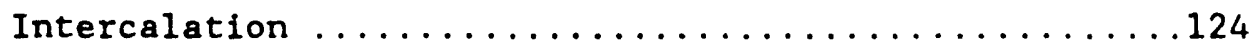

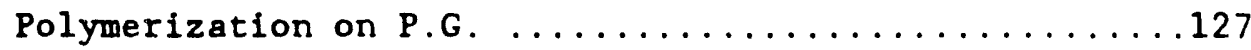

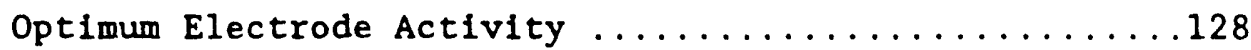

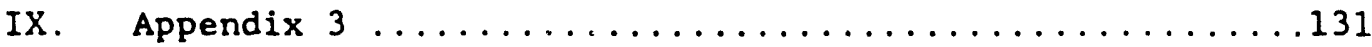

Area Determination - Diffusion Dependent Techniques

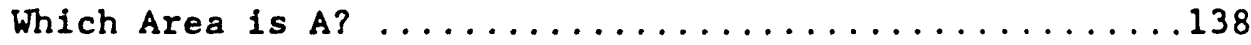

Is the Viscosity of Acetonitrile a Problem? .......140

Is Ferrocene a Reversible Couple? .............143

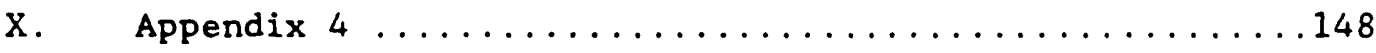

OCP Dependence on Electrode Pretreatment

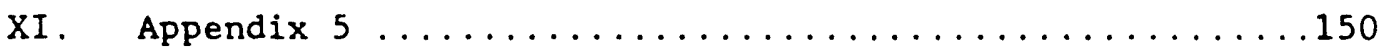

Derivation of Fitting Function

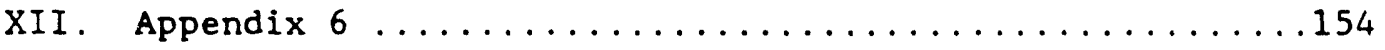

CAPFB - simplex computer program

XIII. References ............................ 173 


\section{List of Figures}

Figure 1. Electric Double Layer Structure $\ldots \ldots \ldots \ldots \ldots$

Figure 2. Electrocapillary Curves $\ldots \ldots \ldots \ldots \ldots \ldots$

Figure 3. Electrochemical Cell ................11

Figure 4 . Working Electrode $\ldots \ldots \ldots \ldots \ldots \ldots \ldots$

Figure 5. Working Electrode Holder ................ 14

Figure 6. Vacuum Heat Treatment Apparatus ...........24

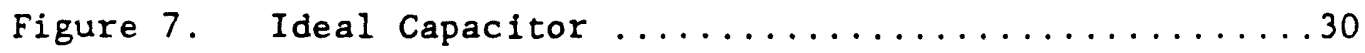

Figure 8. Saw Tooth Waveform $\ldots \ldots \ldots \ldots \ldots \ldots \ldots \ldots \ldots \ldots \ldots \ldots \ldots \ldots$

Figure 9. Discontinuities Plot .......................

Figure 10. Charging/Discharging Curves; Four Cases .......44

Figure 11. Soffer's Deconvolution ..............45

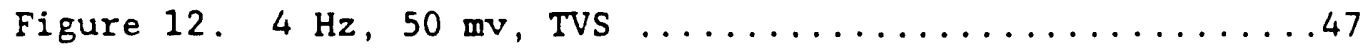

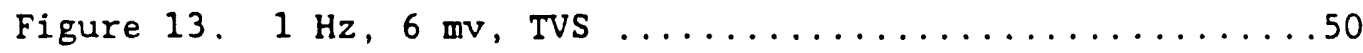

Figure 14. Asystantt's Best Fit ................. 54

Figure 15. Double Exponential Fit ...............56

Figure 16. Questions about the Fit $\ldots \ldots \ldots \ldots \ldots \ldots \ldots$

Figure 17. Fitting the Whole C/D Curve Simultaneously .....61

Figure 18. Comparison between Fitting Functions ........63

Figure 19. Comparison between Fitting Methods ........667

Figure 20. Reproducibility of C-E Curves ..........68

Figure 21. Typical vs Low Background Currents .........74

Figure 22. C/D Curves: Four Cases ..............76 
Figure 23. Typical vs VHT Background Currents ..........78

Figure 24. C-E Curves and corresponding I-E Curves ......79

Figure 25. C-E Curves and corresponding I-E Curves Part II .82

Figure 26. C-E Curves for $0.4 \mathrm{M}$ vs 0.1 M Solutions ......85

Figure 27. C-E Curves and corresponding I-E Curves ......86

Figure 28. Resistances as a Function of Potential .......88

Figure 29. Resistances as a Function of Potential Part II . 90

Figure 30. Frequency Dependence of the Capacitances .......94

Figure 31. Frequency Dependence of the Capacitances Part II 95

Figure 32. Mystery Peak ......................... 102

Figure 33. oxygen Functionalities ................... 104

Figure 34 . Stainless steel CV ..................... 107

Figure 35. What is Apparent: Series of $\mathrm{CV}(\mathrm{s}) \ldots \ldots \ldots \ldots 110$

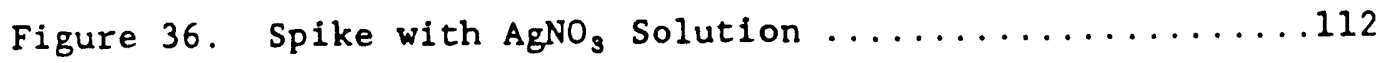

Figure 37. New Reference Electrode Junction ............ 114

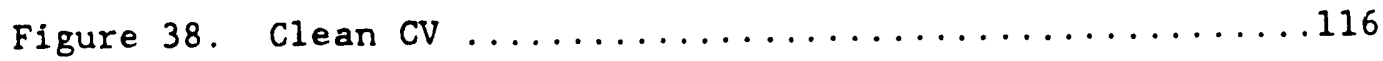

Figure 39. Applied Potential Limits on Graphite Electrodes .120

Figure 40. Applied Potential Limits on Pt Electrodes ......122

Figure 41. Stacking Sequence in P.G. ................ 126

Figure 42. Monomer vs Supporting Electrolyte Solution ......129

Figure 43. Chronoamperometry Input Waveform ...........132

Figure 44. Histogram of Electrode Areas .............. 137

Figure 45. Viscosity Dependence of $i t^{1 / 2}$ for different Non-

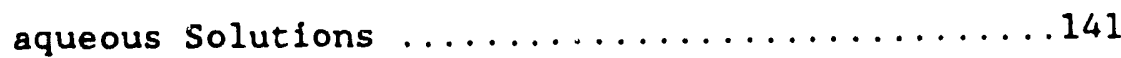

Figure 46. Behavior of $1 t^{1 / 2}$ for Acetonitrile found Here ...142 
Figure 47 . Ferrocene Film Formation ................. 145

Figure 48. Histogram of Open Circuit Potentials .........149

Figure 49. Input Signal and Equivalent Circuit .........151

$$
\text { List of Tables }
$$

Table 1. Trends seen in Potential Instability and Current Noise with a Platinum Working Electrode ........38

Table 2. Trends seen in Potential Instability and Current Noise with a Graphite Working Electrode ........39

Table 3. Intercalate Sandwich Thickness $d_{s}$ for Many Graphite Intercalation Compounds ...........72 


\section{Acknowledgements}

I would like to acknowledge Professor Takanobu Ishida for his guidance, and essential discussions, throughous all of the stages of my graduate research and for his honest and valuable criticism with regard to my career goals.

Many thanks are given to Mr. Leonard Krebs for his endless help, advice, and assistance (if $I$ have achieved anything it is because I stood on the shoulders of giants) and to Ms. Pat Jayanta for establishing a solid foundation, which enabled me to build a further understanding of the system under study.

A debt. of gratitude is also owed to Ms. M. Victoria Kinney for tolerating the insanity brought on by three and one half years of graduate school and for providing the emotional support and friendship which enabled me to endure it.

I am also grateful to: The Department of Energy for their support of this research and for their continued support, Elsevier Sequoia, for permission to include Table 3 and Figure 11 in this thesis; Marcel Dekker Inc, for permission to include Figure 45; The American Chemical Scciety, for permission to include Figure 2; and John Wiley and Sons Inc, for permission to include Figure 37. 


\section{INIRODUCTION:}

PURPOSE OF THIS STUDY

The area of an electrode can be approximated from its dimensions This area is known as the geometric area. However, the surface of a solid electrode has a certain roughness associated with it, which can not be removed. Once a maximum amount of smoothing has been accomplished through polishing the solid electrode, this microscopic roughness remains. This microscopic roughness is absent only on the surface of liquid metals ( 1 ). As a result of surface roughness, the real or true area of an electrode is greater than its frojected or geometric area. The ratio of the true area to the geometric area is called the roughness factor. Few techniques for measuring the true area exist and those techniques which are established are of marginal accuracy. One method which is used to determine the relative roughness of an electrode is based on measuring the capacitance of the electrode in an electrolyte solution.

Measurement of the capacitance can be made using several different techniques, each with parameters and consequent problems complicating the interpretation of the results. In spite of the problems, the measurement can be made and with the 
additional advantage that it can be made in the solution you want to do your experiment in, as opposed to having to change solutions after the area measurement has been made. A technique for measuring the true area of graphite electrodes using capacitance measurements is not yet established but may be possible. This possibility was the ambition behind this study of the capacitance of the edge plane of pyrolytic graphite electrodes in AN solutions.

Other methods for measuring the true area suffer from at least one of three disadvantages: they yield an area closer to the gecmetric area than to the true area; they are destructive, (the act of measuring the area alters the electroce surface); and or they are performed under conditions different from those the electrode will be used in (2). For example, one of these techniques which is performed in an environment different from that in which the electrode will be used, is BET area determinations. These measurements are made in a $N_{2}(g)$ environment at reduced pressures, measuring the amount of $\mathrm{N}_{2}(\mathrm{~g})$ adsorbed. Another technique, diffusjon dependent electrode area measurement, was attempted in this work but found to yield something approximating the geometric area, see appendix 3 .

EIECTRIC DOUBLE LAYER STRUCTURE 
An electode in an electrolyte solution has, at the boundary between it and the electrolyte solution, an interface region which is formed due to the anisotropy of forces where the two phases meet. The term double layer is used to describe this interface. Double layer; $d l$, is a name left from a time when the interface was believed to be simply two sheets of opposite charge, one on the coiductor or semiconductor surface and the other close to the surface, in the electrolyte solution. The interface is now known to be more complicated but the term dl or electrical double layer; edl, has been kept. It is this edl that acts as a capacitor at an electrode, similarly to a parallel plate capacitor.

The electrical double layer may consist of: a layer of negative charges, cathode, or positive charges, anode, at the surface of the electrode; an inner layer. (in the solution), of ions, at the surface of the electrode, solvated and or unsol. vated; and a diffuse double layer, in the solution, consisting of an ionic atmosphere in which ions of one sign are in greater concentration than those of the other. The inner layer consists of two Helmholtz planes, "the locus of electrical centers of a layer of adsorbed ions", called the Inner Helmholtz Plane; IHP, (not always present); and, "the locus of the electrical centers of the hydrated or solvated ions in contact with the electrode surfacen, the Outer Helmholtz Plane; OHP, (see figure 1), (3). 


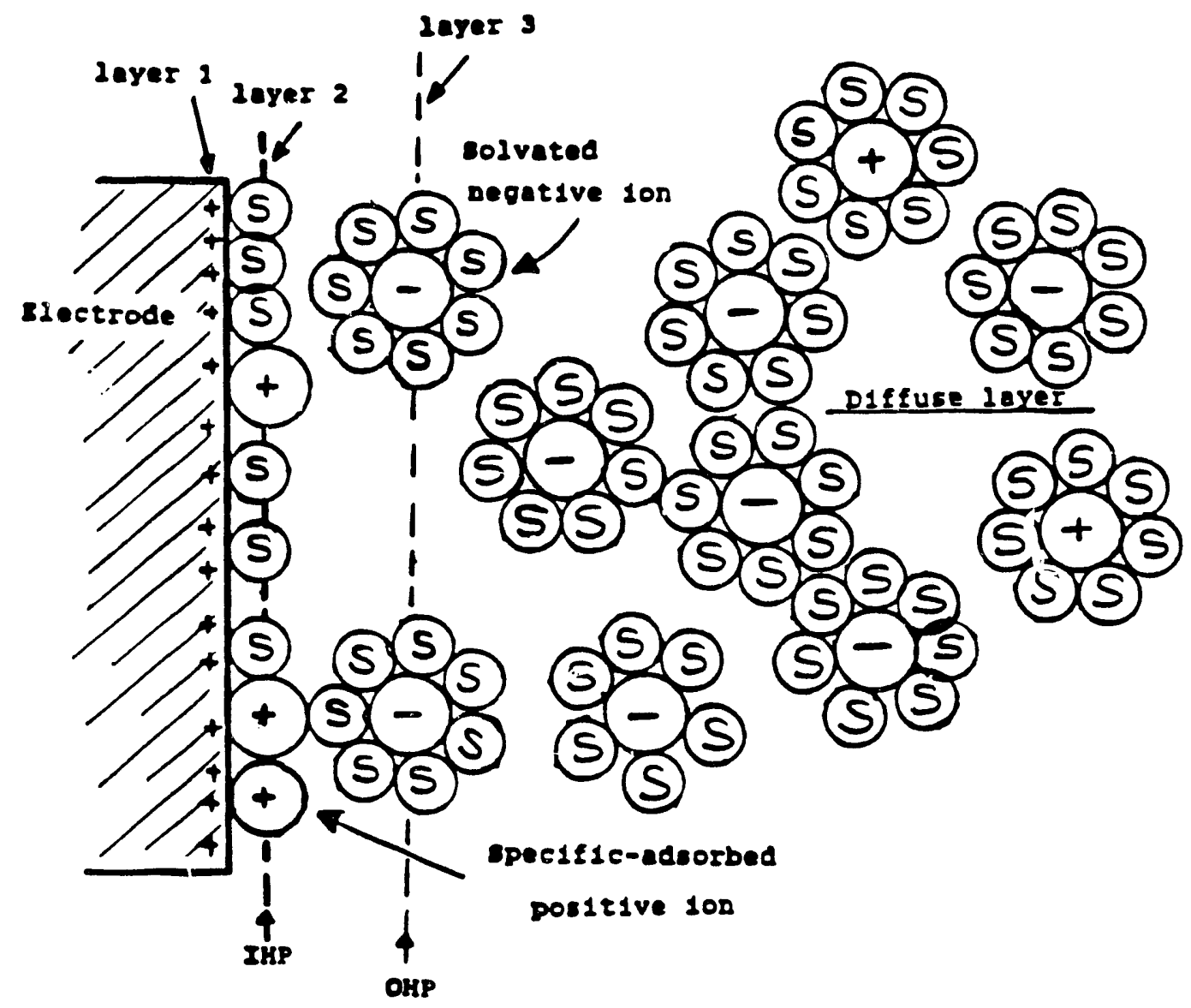

Figure 1. Electric Double Layer Structure

The electric double layer, in the case of a anode, is currently understood to have this structure. 
The edl in a concentrated electrolyte solution has a thickness of raly a few angstroms and since the dimensions of the microscopic roughness are greater than this, the edl follows the contours of the surface. This means that the dl capacitance will be proportional to the electrode surface area making it possible for this method to be used for true area determination.

\section{IDEAL POLARIZED ELECTRODE}

In order measure the capacitance it must be assumed that the current which flows is used to charge the edl and that no charge crosses this interface. An electrode which meets this condition is called an ideal polarized electrode. In other words, there is no species which takes up or gives up electrical charge from or to the electrode. No faradaic current flows and an additional condition is that this is true over a wide range of applied potentials. For this research, using Pyrolytic Graphite, which is an intrinsic semiconductor, it was believed that this condition could be met.

This was thought to be the case since the overpotentials using semiconductor electrodes are usually higher than those at metal ones. This is due to the fact, that at semiconductor electrodes, as opposed to metal ones, electrochemical reactions are strongly inhibited (4). The inhibition arises as a consequence of the fact that for a semiconductor electrode part 
of the potential drop across the electrode is inside the semiconductor's space charge region and not on its surface as it is for metal electrodes. This situation leads to a smaller potential drop at the solution-electrode interface and consequently, a smaller driving force for the electron transfer reaction.

\section{ELECTROCAPILLARITY}

The charged double layer at an electrode-electrolyte interface affects the interfacial surface tension. Since surface tension is the amount of work required to increase the interfacial layer area by a unit amount, and each layer is composed of charges of like sign with repulsive forces between these like charges, then the more charge that exists in a layer of the interface, per unit area, the less work that is required to increase the surface by a unit amount. In other words, the surface tension is decreased because these charges want to get away from each other (1). The work required, or the surface tension, reaches a maximum when there is no net charge in the layer and this is known as the electrocapillary maximum; it occurs at the potential of zero charge; fzc.

The early study of the charged interface was called electrocapillarity. The measured quantity in these studies was the interfacial surface tension, measured as a function of potential, 
and the curves obtained of surface tension as a function of potential, are known as electrocapillary curves, see figure 2 (3). These measurements were made with a Lippman electrometer, which measures surface tension. The separate curves for figure 2 represent the measured surface tensions, at a mercury electrode, in the aqueous supporting electrolyte solutions of the salts that the curves are labelled with. Here, the potential scale is rational potential, which refers to a scale that takes as zero of potential, the potential of the point of zero charge for a NaF supporting electrolyte solution.

At constant solution and electrode composition (designated by the subscript $\mu$ ) and constant temperature and pressure, the change in the interfacial surface tension; $\sigma$, with potential; E, is given by:

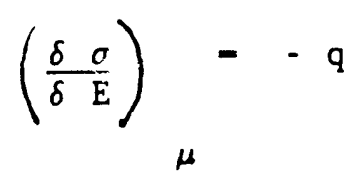

This is the Lippman equation. In words it means that the slope of the electrocapillary curve is equal to the electric charge density, $q$, of the electrode surface. This can be rearranged to $q$ - $-\delta \sigma / \delta \mathrm{E}$ and then differentiating both sides of the equation $\mathrm{C}=$ $\delta q / \delta E=-\delta^{2} \sigma / \delta E^{2}$. 


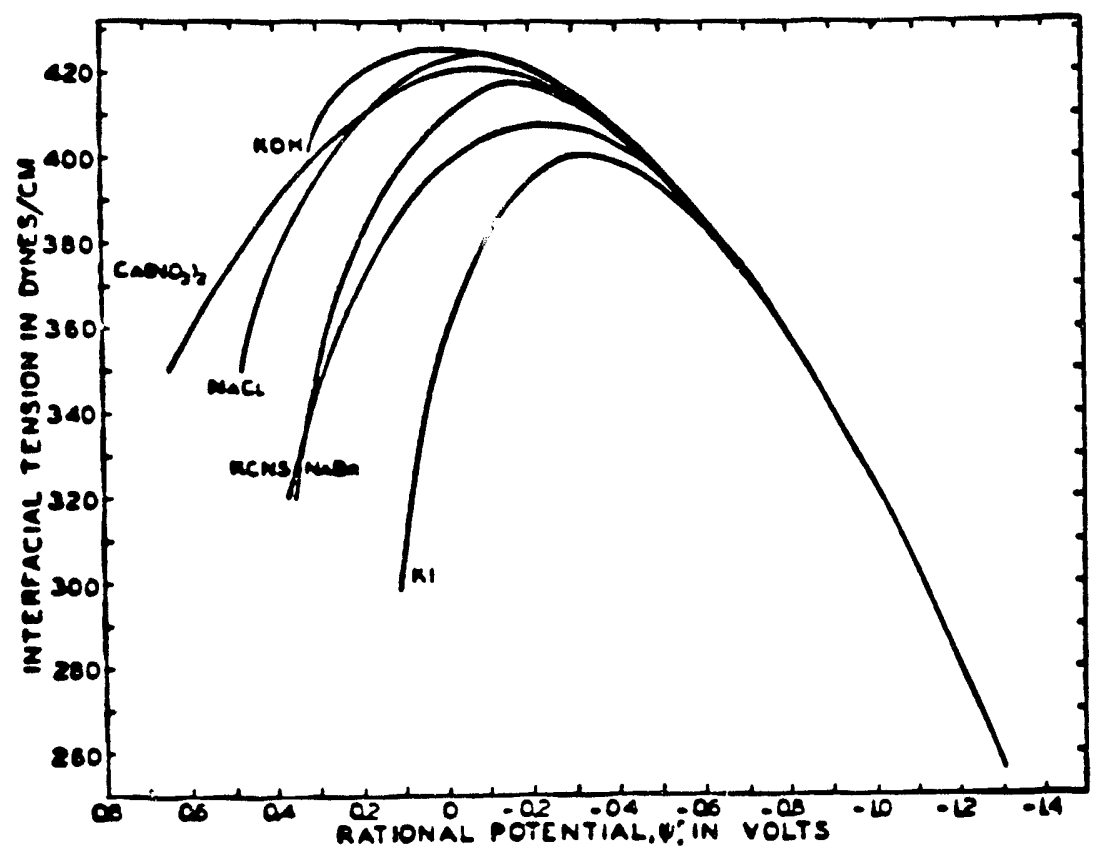

Figure 2. Electrocapillary Curves

Shown here are plots of surface tension vs rational potential for six different supporting electrolytes in aqueous solution. The working electrode was a dropping mercury electrode. Reprinted with permission from Grahame, D.C. Chem. Rev. 1947, 41, p 441. Copyright (1947) American Chemical Society. 
The potential of zero charge; pzc, is the potential of the electrocapillary maximum and is not necessarily equal to zero. The capacitance of the double layer $C_{d l}$ varies with potential with a minimum near the pzc (5).

Electrocapillarity was originally used to study mercury electrodes and the electrometer is best suited for the study of liquid metal electrodes, such as mercury. Measurements can be made on solid electrodes by measuring contact angles but these measurements are difficult. Therefore, this method is unimportant as a means of measuring the capacitarce of solid electrode interfaces.

\section{INTEGRAL VS DIFFERENTIAL CAPACITANCE}

For a capacitor composed of two corductors separated by a dielectric, for example a parallel plate capacitor, the ratio of $q$ to $E$ is constant, or in other words the capacitance $C$ is constant. The capacitance of the edl; $C_{d l}$, however, is not constant ( 3$)$. $C_{d l}$ varies with the d.c. potential imposed across it (3). Otherwise, the electrocapillary curves mentioned before would be perfect parabolas and the first derivative plotted against $E$, would be a line with slope equal to the capacitance $\delta$ ${ }^{2} \sigma / \delta E^{2} ;$ a constant.

The capacitance defined earlier was the differential capacitance; $C_{d}$, or the second derivative of the interfacial 
surface tension with respect to potential at any point along the electrocapillary curve. For a plot of $q$ vs $E$ the differential capacitance; $C_{d}$, is the slope of the resulting curve at a given point but the integral capacitance is the slope of the chord drawn from the origin (the point of zero charge) to the point (3). The integral capacitance is denoted $K ; K--q / E$, and the ratio is a constant with E. For electrodes the capacitance of interest is $C_{d}$.

\section{EXPERIMIENTAL SECTION:}

\section{Electrochenical Cell}

The electrochemical cell used in all the experiments reported herein is shown in figure 3. This cell was crafted by the Department Glass Shop. Three of the upper ports are intended for the auxiliary electrode, reference electrode, and a port for an $\mathrm{N}_{2}(\mathrm{~g})$ inlet. The fourth port is extra, for the possibility of a mechanical stirrer. All electrodes, electrode compartments, and accessories are attached to the cell with Ace Glass Teflon ferrules and bushings. 


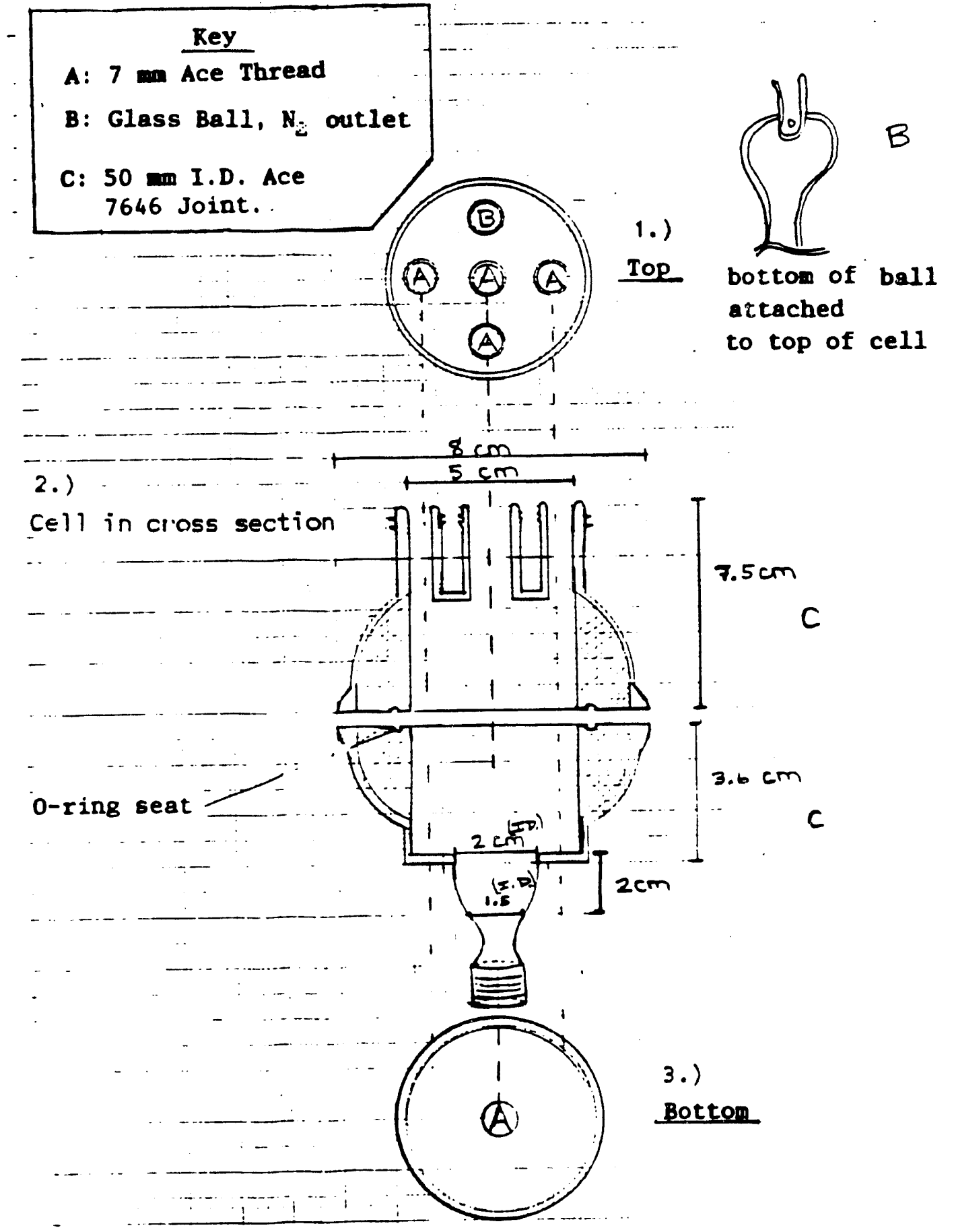

\section{Figure 3. Electrochenical Cell}

(1) Top view, showing the four upper ports. (2) Cross sectional view. (3) Bottom view. 
The bubble shaped glass bulb is the outlet for $\mathrm{N}_{2}$ gas. The cell top is fitted to the cell bottom with a Teflon 0-ring which sits in the seat shown in figure 3 . The cell bottom has one port, for the working electrode and the two halves of the cell sit in an iron ring and are held together with a fork clamp.

\section{Electrodes}

The working electrode is the edge plane of a piece of pyrolytic graphite; $P G$, cut from an originally $5 " x 5 " x: 14 "$ PG plate, manufactured by, and purchased from, Union Carbide, Cleveland, Ohio. The piece was cut into the shape of a rectangular prism of dimensions $1 / 4^{\prime \prime} \times 1 / 4^{\prime \prime} \times 3 / 4^{n}$, see figure 4 , using a Low Speed Diamond Saw, model \#650 purchased from South Bay Technology Inc of Temple City, CA. The resistivity of the pyrolytic graphite was $5.0 \times 10^{-4} \mathrm{Ohm}-\mathrm{cm}$ in the a direction and $0.5 \mathrm{Ohm}-\mathrm{cm}$ in the c direction (6), see figure 4. This electrode was held in an electrode holder consisting of three parts: hood, stem, and stainless steel insert (figure 5a, and b, respectively), all designed by the Ishida group and machined by the Department Machine Shop. The hood and stem were cut from a Kel-F rod, purchased from AIN Plastics Inc., Mount Vernon, NY. The electrode sat in the $3 / 8^{\prime \prime}$ diameter hole of the stem shown in figure $5 b$, on top of a stainless steel insert for electrical contact and the hood was tightened down on this making a liquid 


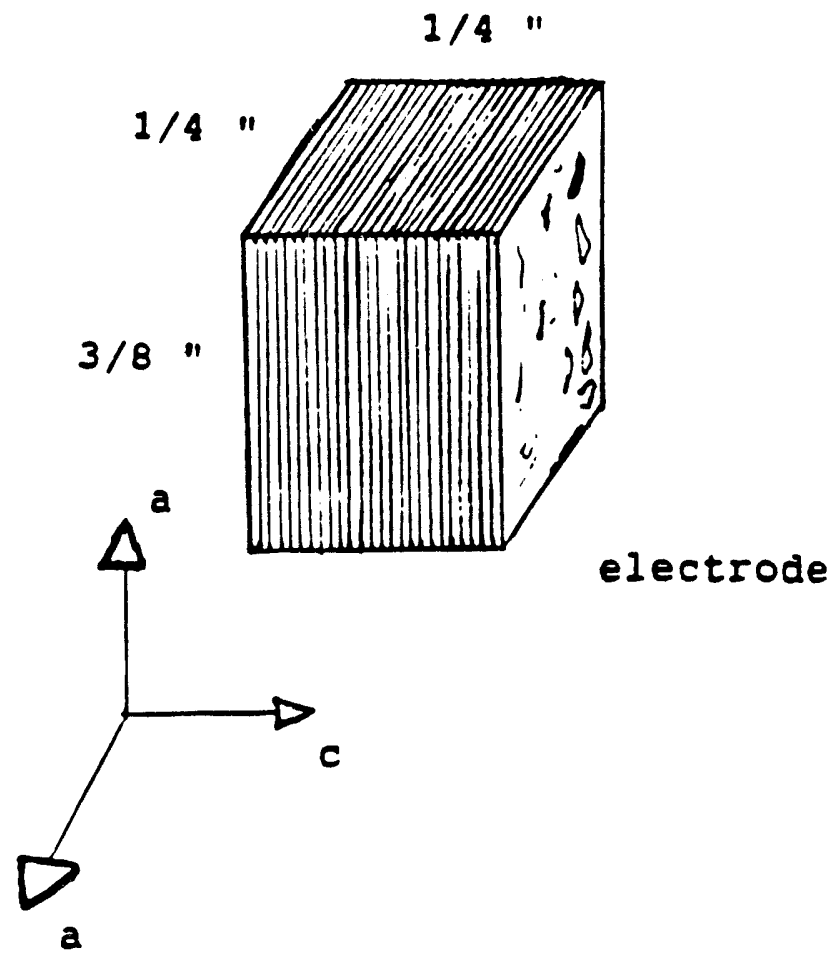

Figure 4. Working Electrode

The dimensions of the working electrode and their orientation with respect to the anisotropic structure of the pyrolytic graphite. 

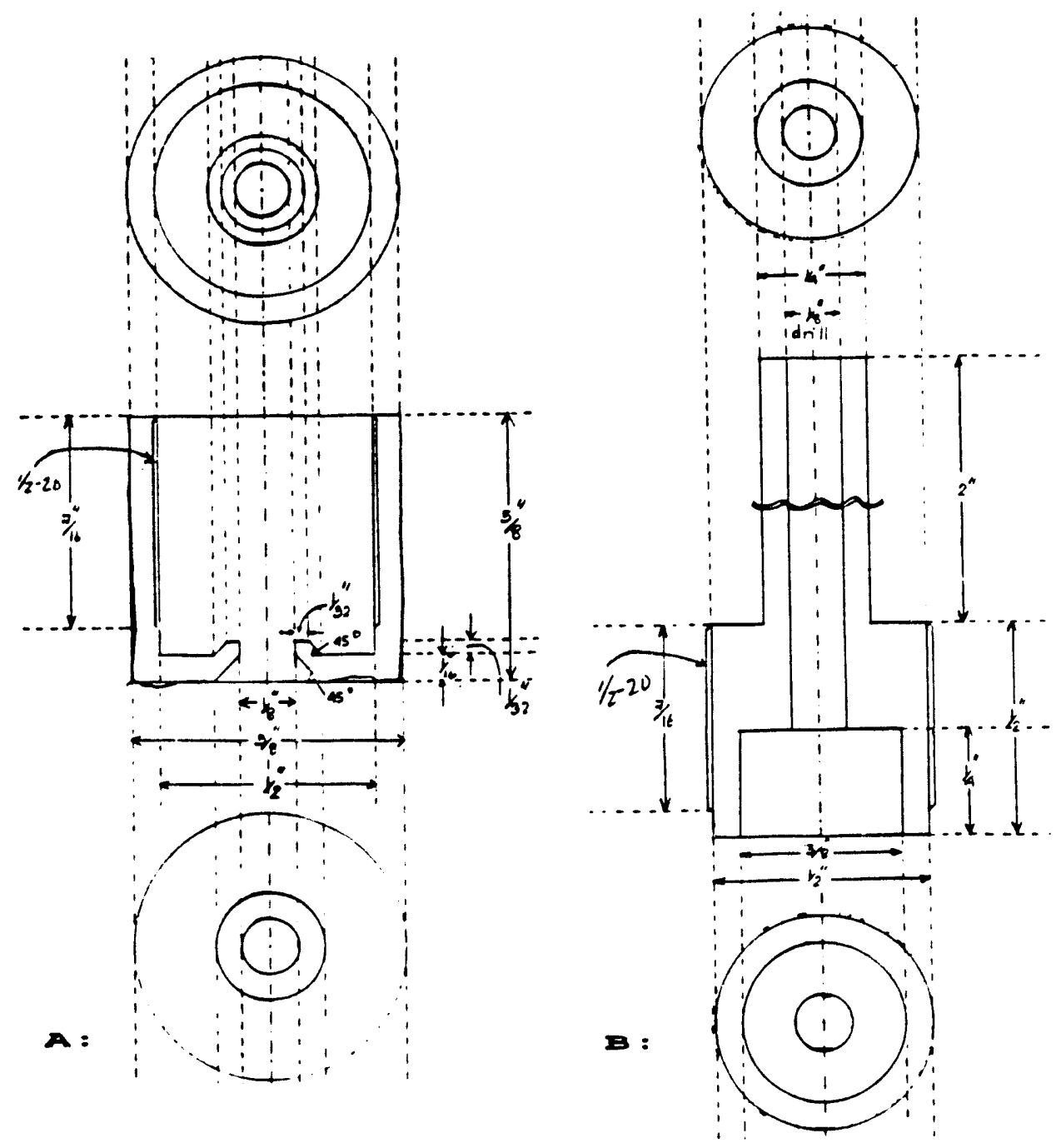

Figure 5. Working Electrode Holder

(a) The Hood of the electrode holder shown in cross section. (b) The stem of the electrode holder is shown here in cross section. The electrode shown in figure 4 sits in the $3 / 8$ "opening in the stem on top of a stainless steel insert (not shown). The hood is then tightened down on this. 
tight seal. When in place, a $1 / 8^{n}$ diameter circular region of the edge plane of the working electrode was left exposed by the electrode holder.

The auxiliary electrode consists of a $7 \mathrm{~cm}$ length of $0.5 \mathrm{~mm}$ diameter platinum wire purchased from Johnson Matthey Chemicals Ltd, Royston, England. The purity of this wire was 99.99858. The wire was coiled into a helix of approximately $1 / 2 \mathrm{~cm}$ diam ter, attached to a tungsten lead, for electrical contact, and placed in a $7 \mathrm{~mm}$ OD pyrex tube with a uranium glass seal at its end, below the W/Pt jur.ction.

This electrode had one of two possible fates; it was either put directly into the cell by attaching it to one of the ports or it was attached to a separate compartment which was attached to - ie cell through one of the ports. The separate compartment was made from another Ace 5027 adapter with \# 7 Ace threads to which was attached a short length of $4 \mathrm{~mm}$ diameter pyrex tubing. The 6.nd of the $4 \mathrm{~mm}$ diameter pyrex tubing was sealed with a $4 \mathrm{~mm}$ diameter Vycor tip purchased from EG\&G PARC, Princton, NJ, which was attached with a $1 / 2 \mathrm{~cm}$ length of shrinkable teflon tubing (expanded diameter $0.158^{n}$, recovered diameter $0.124^{\prime \prime}$ ) Small Parts $\therefore$ Miari, Florida. The tubing was shrunk with a heat gun. itie vycor tip served as the junction between the main cell " $=;$ artant and the auxiliary electrode compartment. The iriale compartment was filled with the same solution as the a:: coopartment. In later experiments the separate compartment 
was found to be undesirable and the auxiliary electrode was included directly in the cell. This is described further later on.

The reference electrode was a $\mathrm{Ag} / \mathrm{Ag}^{+}$electrode $\left(0.1 \mathrm{M} \mathrm{AgNO}_{3}\right.$; $0.1 \mathrm{M}$ TEATFB; in acetonitrile). This electrode consisted of a 7 mm diameter Pyrex tube, $6 \mathrm{~cm}$ in length, tapering down to $4 \mathrm{~mm}$ diameter pyrex tubing. The tip was sealed with a Vycor tip again attached with shrinkable teflon tubing. Inside the tube was the $0.1 \mathrm{M} \mathrm{AgNO}_{3} ; 0.1 \mathrm{M}$ TEATFB; AN solution and into this was dipped a Ag wire, 99.98 Johnson Matthey Inc., Seabrook, NH. To the silver wire was soldered a nickel coated tungsten wire, for electrical contact. The tungsten wire was then inserted through a small hole in a polypropylene cap and the inside of the cap was filled with Easypoxy covering the soldered joint. This epoxy cement is insoluble in $A N$ and sat well above the solution level. When the epoxy was dry the cap was put on the barrel, and parafilm was wrapped around the joint to ensure that it was airtight.

Later on it was found that the vycor junction leak rate was too high (see appendix 1) and this was therefore changed to a Pt wire sealed in pyrex junction, leak rate $3-30 \mathrm{ul} / \mathrm{hr}$ (7). Unless otherwise stated all potentials will be reported vs the $\mathrm{Ag} / \mathrm{Ag}+$ (0.1 $\mathrm{M} \mathrm{AgNO}_{3} ; 0.1 \mathrm{M}$ TEATFB; AN) electrode, $\mathrm{V}_{\mathrm{Ag} / \mathrm{Ag}^{+}{ }^{-} \mathrm{V}_{\text {sce }}-0.337}$ - $V_{\text {nhe }}-0.565$ (8). The $0.1 \mathrm{M}$ TEATFB, in the reference electrode solution, didn't significantly affect the potential. 
CHEMTICAIS AND MATERIALS USED

The $\mathrm{N}_{2}(\mathrm{~g})$ which passed through the bubbler, entering the cell and, bubbling into the solution, or the space above the solution, (depending upon the conditions desired), was Ultra High Purity Grade, $\mathrm{N}_{2}(\mathrm{~g})$ from Union Carbide. This gas was further purified by a series of steps. It was first passed through a column of activated charcoal 6-14 mesh, (Fischer Scientific), followed by a column of silica gel desiccant, J.T. Baker Chemical Co., Phillipsburg, NJ. These two steps were intended to remove any oil residues and water, respectively, that might have escaped a purification process.

Next, the $\mathrm{N}_{2}(\mathrm{~g})$ was bubbled through concentrated sulfuric acid, to oxidize and remove organics and then through another column of silica gel. The final step was to wet the gas with AN and this was accomplished by bubbling it through a $250 \mathrm{ml}$ trap filled with AN (purity and work up described below), to which several grams of $\mathrm{CaH}_{2}$, Baker Chemical Co., practical grade 948 , were added as a drying agent.

Any and all acetonitrile, HPLC grade Fisher Scientific, Fairlawn, $\mathrm{NJ}$, was further purified by distillation over $\mathrm{CaH}_{2}$, under a $\mathrm{N}_{2}(\mathrm{~g})$ atmosphere. The $\mathrm{N}_{2}(\mathrm{~g})$ used here was treated only to the extent of the first silica gel column described above.

The supporting electrolyte solutions used in the electrochemical cell were prepared from the acetonitrile described above 
and from tetraethylammonium tetrafluoroborate (TEATFB) $99+8$ pure, purchased from Alpha Chemicals of Danvers, MA. The salt was stored in its container in a desiccator and the time outside of the desiccator was minimized, to avoid adsorption of water by the salt.

The $0.1 \mathrm{M} \mathrm{AgNO}_{3}$ solution used in the reference electrode was purchased from Koslow Scientific Co. of Edgewater, NJ.

\section{INSTRUMENTATION, HARDWARE AND SOFTWARE}

Two different potentiostats were used in these experiments. One was a EG\&G PAR model\# 362 Scanning Potentiostat and the other an IBM Voltammetric Analyzer EC/225. Both instruments were interfaced with an IBM PC, AT through a high performance analog and digital I/O board manufactured by Data Translation Inc., Marlborough, Mass. This in combination with Asystant+ Data Acquisition Software, purchased from Macmillian Software Co. N.Y., N.Y., made data acquisition with the computer possible. For some preliminary experiments, described in the appendices, data was acquired with an XY Recorder Series 200, purchased from The Recorder Co., San Marcos, Tx.

The EG\&G PAR potentiostat was used for the preliminary work described in the appendices and the IBM potentiostat was used for all subsequent work described in the main body of the text. Both of these instruments scan in 1 mv steps (i.e. with $1 \mathrm{mv}$ re- 
solution). The accuracy of the applied potential for the EG\&G potentiostat is $\pm 3 \mathrm{mv} \pm 0.4$ of the initial potential setting and the accuracy of the measured current is \pm 0.4 of the current range setting.

For the IBM instrument the accuracy of the applied potential is \pm 0.58 of the applied value and the accuracy in the measured current values is \pm 1 \& of the value.

The A/D, I/O board has an accuracy of $\pm 0.1 \&$ full scale reading and an input impedance of $100 \mathrm{M} \Omega$.

For generating the triangular waveforms of varying frequencies a waveform generator was used which was first passed across a voltage divider, $100 \mathrm{k} \Omega$, and then hooked up to the auxiliary input BNC connector of the IBM potentiostat. The waveform generator was a Dana Exact waveform generator model \# 120, manufactured by Dana Exact Electronics, of Hillsboro, Oregon. It had the follnwing specifications: output impedence: 50 $\Omega$, frequency accuracy \pm 2 of frequency range, amplitude stability $0.05 \&$ of $\max P-P$ amplitude for 10 minutes.

\section{Electrode Pretreatment Procedures}

Polishing of the edge plane, graphite electrode was necessary in order to: expose a fresh uncontaminated surface, to obtain a relatively smooth surface, and because it is believed to activate the surface ( 9 ). Polishing was performed on two optical 
flat plates purchased from Harrick Scientific Co. of Ossining, N.Y. One plate was for course polishing the other for two grades of fine polishing. Polishing was done in three different slurries each containing a different grade of diamond powder; $d p: 1-2$ um, $1 / 2-1 \mathrm{um}$, and $1 / 4-1 / 2$ um dps. These were purchased from Kay Industrial Diamond Co., Deerfield Beach, Fl. Each grade of dp was mixed with distilled $\mathrm{H}_{2} \mathrm{O} ; \mathrm{dH}_{2} \mathrm{O}$, in a polypropylene bottle at a ratio of $1 / 2 \mathrm{gram}: 100 \mathrm{ml}$ of $\mathrm{dH}_{2} \mathrm{O}$

After a PG electrode was cut it was placed in a $50 \mathrm{mI}$ beaker filled with $\mathrm{dH}_{2} \mathrm{O}$. The beaker was then clamped into place in a Ultra Sonic Cleaner model\# 8850, purchased from Cole-Parmer, of Chicago Illinois, and this was run for 3 mins. The $\mathrm{dH}_{2} \mathrm{O}$ in the beaker became filled with graphite dust deposited during cutting, and so the water was changed and the above step repeated.

When polishing was done finger cots were worn on all fingers and thumbs which came in contact with the electrodes. Polishing was started with the 1-2 un dp slurry on the course optical flat. Each electrode was polished several times applying a small force and a circular motion to the electrode. In between polishings the flat was rinsed off and dryed with kimwipes. The position that the electrode was held in was rotated in order to apply a more uniform polishing. After a total of approximately 60 rotations the electrode was rinsed off with $\mathrm{dH}_{2} \mathrm{O}$ and again sonicated for approximately 3 mins, this was to remove graphite material and the previous grade of $d p$. It was important to remove the other 
grade of dp from the electrode because mixing them could cause scratching of the surface. These steps were repeated exactly for all three grades of dp except the second two grades of polishing were done on separate halves of the second plate. After polishing the electrodes were dried by one of the methods to be described in the optional pretreatment procedures section.

\section{Optional Pretreatment Procedures}

All of the pretreatment procedures described below were conducted only where specified in the text. Previous procedures were always performed unless specified in the text.

\section{Drying}

Drying the electrodes after polishing was accomplished by several different techniques, including: letting them stand at room temperature exposed to the air of the lab, drying under a vacuum of a few mtorr for approximately 12 hrs, drying at ambient pressure in an oven, or drying at $600 \mathrm{C}$ and $10^{-6}$ torr; known as Vacuum Heat Treatment; VHT.

For drying in air, the electrodes were simply allowed to stand in a loosely covered beaker at room temperature and pressure for a day or so before being used. This is the least effective way of drying and it is believed that, if electrodes 
are exposed to open air for a few hours after polishing, they become deactivated.

For drying in the oven, electrodes were used immediately after having been polished and dried in a Sargent Welch oven at $90 \mathrm{C}$ for four hours. This probably deactivates the electrodes also.

For drying under vacuum the electrodes were patted dry on all surfaces with a kimwipe, except for the polished edge plane surface and stacked in a pyrex thimble with teflon ferrules in between them to protect their polished surfaces. The thimble was then evacuated down to $\leq 10$ mtorr with a mechanical pump, Welch Duo-seal model \#1400, as measured with a thermocouple gauge model \# TG-7, Veeco Instruments Inc., Plainview, N.Y.. Between the pump and the vacuum line, a dry ice trap was maintained. The graphite was kept at this pressure for at least $12 \mathrm{hrs}$ and otherwise until used.

\section{Vacuum Heat Treatment}

Vacuum Heat Treatment, or VHT, besides being the most effective means of drying the electrodes, is used as a way of removing surface oxides from the surface of the edge plane of the PG (10). The apparatus used for this treatment is shown in figure 6. This method consisted of placing approximately 6 graphite 
electrodes, (max capacity 12), In a quartz tube (OD: 1/2n, and ID: $3 / 8^{\prime \prime}$, length: $40 \mathrm{~cm}$ ), attaching this to the bellows valve via Cajun fitting 1 , sealed with an ungreased Neoprene 0-ring, (see figure 6), and pumping the tube and lines down with the mechanical pump for approximately 3 hrs or until a vacuum of $\leq 1$ mtorr is achieved. Once this level of vacuum had been reached, the liquid $\mathrm{N}_{2}$ trap was filled. At this point the cooling water for the diffusion pump, Consolidated Vacuum Corporation of Rochester, $N Y$, is turned on along with its heat source, the roughing valve is closed, and the foreline valve is opened, see figure 6 . The Ionization Gauge, Joule Degas model \# RG-81, Veeco Instruments, Plainview, $N Y$, is now turned on and allowed to warm up. After about 10 mins, it is calibrated and then the power to the filament is turned on. The thermocouple gauge is now turned off. Once the meter reads a vacuum of $\leq 1 \times 10^{-6}$ torr, the oven, Lindberg Heviduty, is turned on and allowed to slowly heat up by setting it first at a low setting. Approximately one hour is needed to achieve $600 \mathrm{C}$, during which time the pressure has to be carefully monitored so that it doesn't exceed $1 \times 10^{-6}$ torr. The vacuum is controlled by adjusting the variac and the flow of water to the cooling coil. Also, the level of $\mathrm{N}_{2}(1)$ in the cooling trap has to be monitored. 


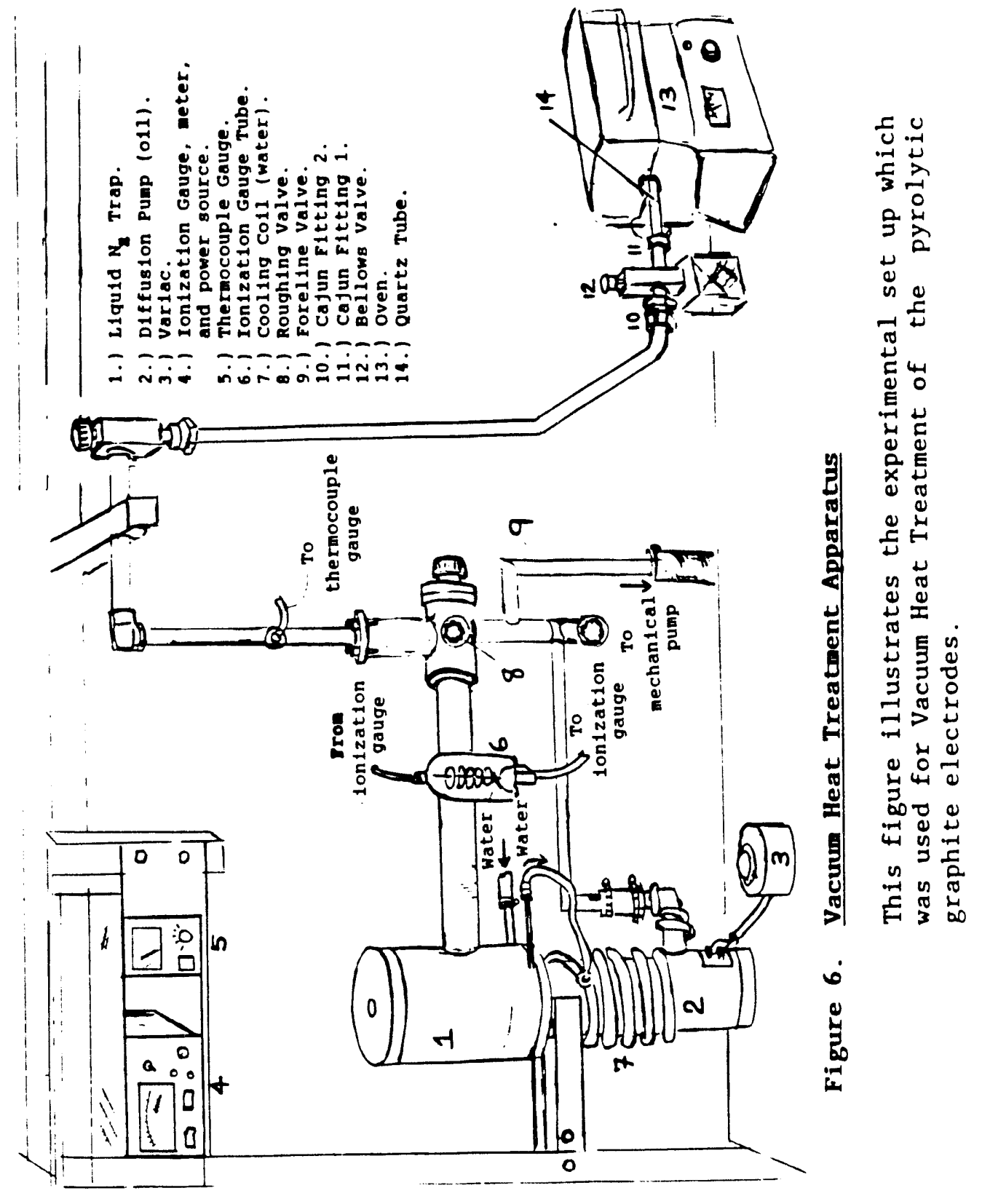


The pressure is critical because if it becomes too high, the graphite could undergo some degree of combustion. After 20 mins of $600 \mathrm{C}$ temperatures, at high vacuum, the oven is turned off and allowed to cool. Once a temperature of $300 \mathrm{C}$ is reached the oven door is opened, exposing the outside of the quartz tube to open air and thus accelerating the cooling rate. During this time the pressure is maintained at $\leq 1 \times 10^{-6}$ torr. When the graphite has cooled completely, this requires about 40 mins, the bellows valve connecting the quartz tube with the vacuum line is shut and the valve with quartz tube is disconnected from the vacuum line at cajun fitting 2 , see figure 6 . The tube and valve are then transferred to a glove bag which is purged with UHP, $N_{2}(g)$. The diffusion pump is then turned off and allowed to cool. After 20 mins the ionization gauge is turned off and the thermocouple gauge turned back on. The cooling water remains on until the diffusion pump is cool to the touch, at which time the roughing valve is opened and the foreline valve closed.

The graphite is now in the quartz tube with the bellows valve shut, and in the glove bag. At this point all the necessary equipment for cell assembly including: cell, electrodes, Teflon 0 -ring, and solutions are placed in the glove bag which is purged several times with UHP, $N_{2}(g)$ (this $N_{2}(g)$ having received the same treatment as the $\mathrm{N}_{2}(\mathrm{~g})$ for $\mathrm{AN}$ distillation). The bag is then filled with $\mathrm{N}_{2}(\mathrm{~g})$ and completely sealed. The working electrode is assembled in the cell bottom, supporting electrolyte solution 
added, and the cell is assembled all under an $N_{2}(g)$ atmosphere. The quartz tube, still contalning the other graphite pieces is then attached back to the valve and the valve shut to keep the remaining graphite pieces under an inert $N_{2}(g)$ atmosphere. The cell is then removed from the glove bag and set up in the hood and $\mathrm{N}_{2}(g)$ is immediately bubbled through the inlet into the solution.

\section{Radio Frequency Plasna Treatment}

In this pretreatment procedure electrodes are polished in the usual manner and then patted dry with a kimwipe on all surfaces except the polished edge plane. They are then placed :nside of the chamber of a r.f. plasma generator; Harrick Plasma Cleaner Model\# PDC-23G, Harrick Scientific of Ossining, NY. This chamber is then pumped down using a roughing pump, with a dry ice trap in line between them. After a vacuum of a few mtorr has been achieved and the chamber has sat at this pressure for 3 hours, the line and chamber are purged with 100 mtorr portions of Ultra $\mathrm{High}$ Purity; UHP, $\mathrm{O}_{2}$ gas. This was repeated 3 times. The amount of $\mathrm{O}_{2}$ gas entering the line was controlled with a pressure regulator and by manually opening a vacuum stop cock, while monttoring the pressure on a thermocouple gauge. One final volume of $\mathrm{O}_{2}$ gas was released into the vacuum line achieving a pressure of 300 mtorr and the chamber of the radio frequency plasma gener- 
ator was then isolated from the vacuum line; the chamber containing $a \approx 300$ mtorr pressure of $\mathrm{O}_{2}(\mathrm{~g})$. The radio frequency plasma chamber was then set at a high setting for $1 / 2 \mathrm{hr}$. During this time it glowed a blue color. The radio frequency power in the coil is approximately 18 watts.

After this time the generator was turned off and the needle valve on its front was slowly opened, allowing the chamber to come to room pressure. Once the pressure inside and out were equal, the cover drops off and the graphite was removed and used immediately.

\section{Extractions}

Two extractions were carried as a means of ensuring that the graphite was chemically clean. One extraction was for inorganic substances and the other was for organic substances. These were carried out in two separate soxhlet devices, one filled with $\mathrm{MeOH}$ for inorganic substances and one with hexane for organics. The $\mathrm{MeOH}$ and Hexane were HPLC grade from Fischer Scientific. When these extractions were used it was prior to polishing. 


\section{VHICH MEASUREAENT TBCHNIQUB TO OSE?}

Two capacitance measurement techniques are often used. Measurement with an a.c. impedance bridge and with the triangular voltage sweep method. Randin and Yeager (11-13) use an a.c. impedance bridge to measure the capacitance of the edge and basal planes of pyrolytic graphite electrodes in aqueous solutions and they believe they see a fast and a slow charging capacitance. The slow charging capacitarce is known as a distributed capacitance effect. The speed with which the slow charging process takes place is determined by the resistance involved with ions moving into the internal structure of the electrode. They see the distributed capacitance effect on the basal plane.

They also consider the possibility of one on the edge plane but rule this out. However, they are unable to go to a frequency of less than $100 \mathrm{~Hz}$ and this may be too high a frequency in the case of very small openings such as the microfissures on the edge plane of PG. In other words, the period of the input waveform of $100 \mathrm{~Hz}$ frequency could be much shorter than the time necessary for the slow charging capacitance to even begin charging. According to oren, Tobias, and soffer, (14, p.92), "audiofrequency bridges which are suitable for flat and small surfaces, are in most cases too fast for the time independent $C$ determination of porous electrodes." 
The TVS method consists simply of applying a TVS to the electrode of interest, through the use of a three electrode cell and a potentiostat, and recording the current output measured by the potentiostat, (see figure $7 a$ and $b$ ). Figure $7 a$ is the input TVS and figure $7 b$ is the current response. From this curve and the relation $C-i / \nu$, where $\nu$ is the scan rate, and $i$ the current, the capacitance can be calculated.

Iwo other variables, in addition to the TVS frequency, may affect the amount of internal structure of an electrode which is accessible tc charging. These are the radii of the ions of the supporting electrolyte used and the potential applied. A large poteniial gradient could provide a greater driving force to draw ions into the internal structure (i.e. pores, cracks, or microfissures). Thus, in order to carry out this investigation, it would have been ideal to explore all of these variables. However, the capacitance was determined as a function of potential for only one supporting electrolyte; tetraethylammonium tetrafluoroborate. The variables which were explored are: potential dependence, frequency dependence, supporting electrolyte concentration dependence, and electrode pretreatment dependence.

\section{QUESTIONS OF TECHNIQUE}

The first question was one of technique, how was the potential going to be stepped from one value where a capacitance 


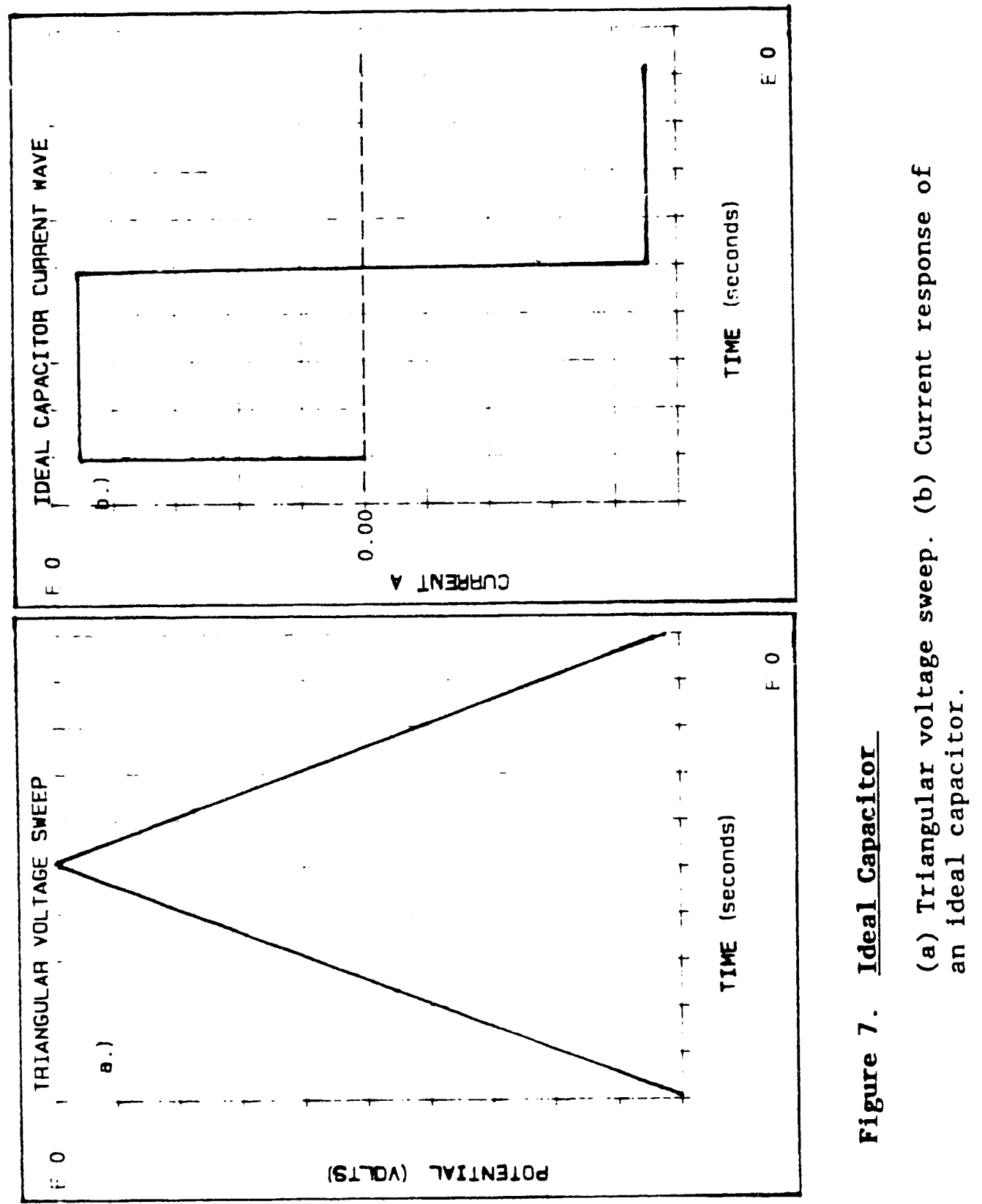


measurement was made to another in a clean and reproducible fashion? Both Soffer and co-workers (14-18), and Gagnon and coworkers $(19,20)$, who do work similar to that which is being attempted here, use a saw-toothed waveform, in which, in the case of Gagnon and co-workers $(19,20)$, the potential is scanned "up" $140 \mathrm{mv}$ at some constant rate and then scanned back $90 \mathrm{mv}$ in the opposite direction at the same rate. $(19,20)$ This results in a net forward step of $50 \mathrm{mv}$. This idea was used in this work but with a few modifications. The forward step was decreased to 100 mv and the backward step to $50 \mathrm{mv}$ resulting in a net step of 50 mv/triangular voltage sweep. Ideally, when a series of these are connected, this yields a clean reproducible way of making capacitance measurements as a function of potential. See figure 8 a for a plot of the input waveform created for this work by constructing the ramp up and the ramp down and then adding them together a specified number of times. The waveform shown in $8 a$ scanned from -0.650 to $0.650 \mathrm{~V}$ and back again in net $50 \mathrm{mv}$ steps, see figure $8 b$, for a plot of the current response.

\section{NOISE IN THE CURRENI RESPONSE}

From the current output in figure $8 \mathrm{~b}$, a problem is apparent. The current output is too noisy to be of use. First, it was thought that maybe the waveform being used was causing the noise. 

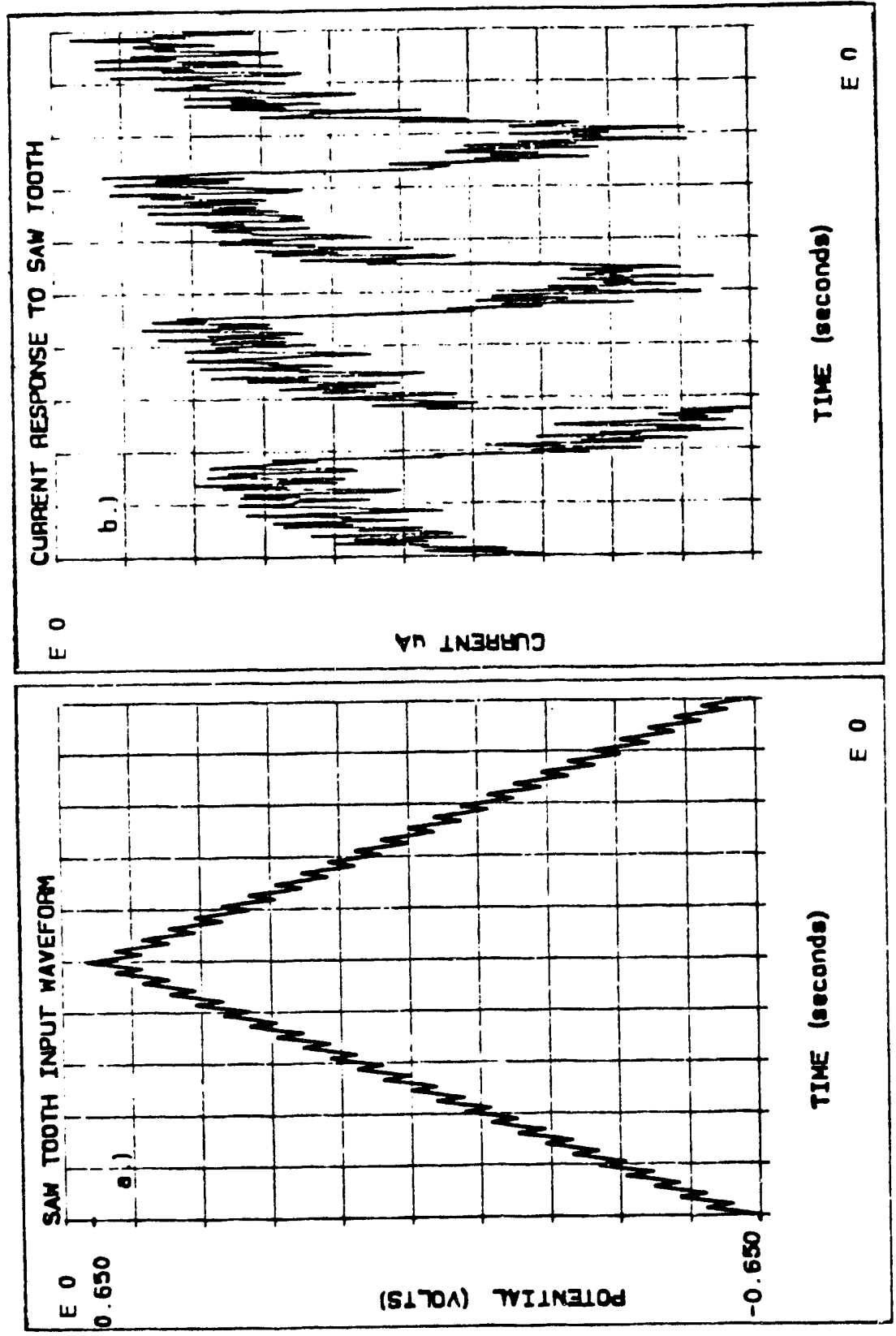

$\stackrel{\circ}{\circ}$

os

ç

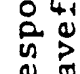

需 3

䓀

正

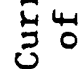

อ

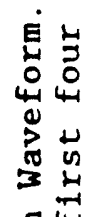

잉

$\leftarrow$

离

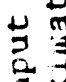

告

$\rightarrow$ 둔

ชิ

$\sum_{0-1}^{\infty}$ 
This input waveform had been created as described above, using Asystant+ Data Acquisition Software. This software was also used to generate the waveform which was sent to the potentiostat, and to collect tire current data measured by the potentiostat (see the experimental section for more details). The waveform which had been used, (figure $8 a$ ), had a ratio of points in the waveform, to mv scanned of $1 \mathrm{pt}: 2 \mathrm{mv}$. This ratio might be too small, having too few points:mv which could lead to discontinuities in the waveform (see figure 9). These discontinuities could produce a similar current output to that produced by the discontinuity in the triangular voltage sweep present at the switching potential. This might look like the noise seen in figure $8 \mathrm{~b}$.

To investigate this possibility, the number of TVS(s) in the input waveform was drastically reduced and correspondingly, the potential range covered was also reduced. This allowed for greater control over the ratio of number of points:mvs in the waveform. A range of ratios from 5pts:mv to 1pt:5mvs were looked at and all appeared to yield the same noisy result. Even potentiostating with no waveform from the computer gave a noisy result.

The IBM EC/225 potentiustat is supposed to scan potentials in lmv steps which is also the instability in a potentiostated potential and could be the cause of the noise in current. When looking at the noise from potentiostated data it was determined that the amplitude of the noise was a few hundred $n A$ peak to peak 


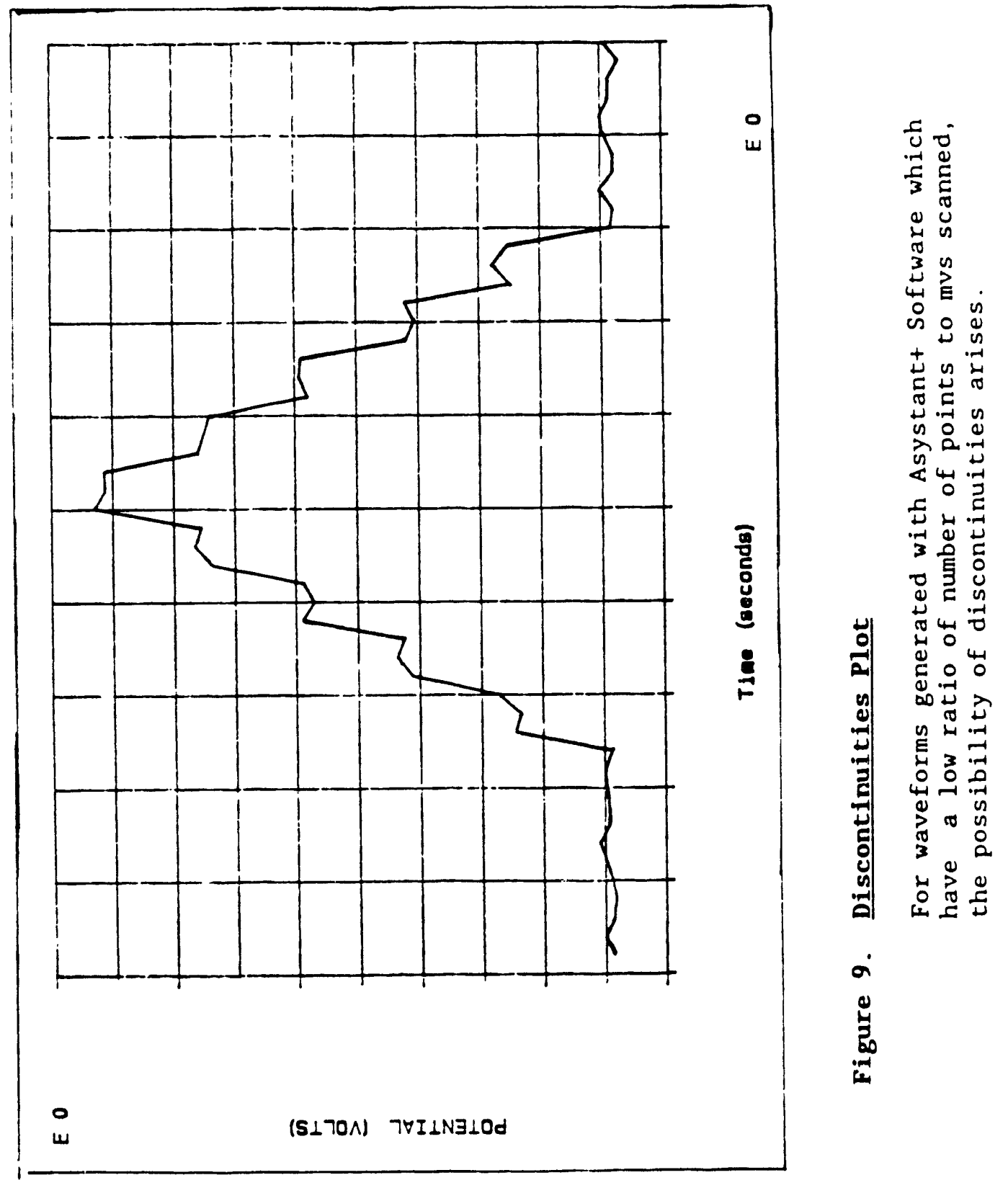


and the frequency seemed to be either $60 \mathrm{~Hz}$ or some integer multiple of $60 \mathrm{~Hz}$. This suggested some form of electrical noise.

DESTABILIZATION OF THE POTBRTIAL

A source could not be found and so as a desperate act, the following was tried. The auxiliary electrode for these experiments had been a Pt wire coiled and dipped into a Pyrex tube, containing supporting electrolyte solution and separated from the rest of the cell by a Vycor tip, sealed on the end of the pyrex tube with shrinkable teflon tubing. As a far fetched idea, it was thought that the use of the separate compartment, with Vycor tip junction, may somehow be contributing to the noise and therefore the auxiliary electrode was included in the same compartment. The result was the same noise with a new additional problem; the potentiostat was now unable to stabilize the potential. This was evident in the large oscillations seen in the potential, which are not shown, since it was believed that this state was bad for the instrument and therefore it was turned off whenever this condition existed.

This was strange because experiments had been conducted before in our lab, with the auxiliary electrode in the same compartment, and the destabilization in the potential had not been seen. However, the relative proximity of the reference 
electode to the working electrode and the auxiliary electrode to the the working electode, turns out to be important.

This had something to do with the response time of the potentiostat. If it was able to respond faster than the time needed for the working electrode to reach the desired potential and for this value to be measured by the instrument, then the instrument would attempt to establish the correct potential by changing that applied. By the time the applied potential had been changed, the potential at the working electrode, measured by the instrument, was that which had originally been desired. Now, however, due to the changed applied potential, that at the working electrode became too big causing the potentiostat to apply a potential less than the original one. This would proceed back and forth resulting in an oscillation in the measured potential.

Even when no destabilization in the potential is seen at normal sensitivities, if a sensitive enough scale is used some can be detected. This makes sense, since the instrument operates through feedback, measuring the potential at the working electrode relative to the reference electrode and compensating if it isn't what it was set to be.

Therefore, it seems that the vycor tip was slowing down or increasing the response time of the feedback loop of the potentiostat, so that oscillations in the potential didn't occur with it in place. The Vycor tip was preventing the normal operation of 
the potentiostat and therefore, it was concluded that it shouldn't be used. Now there were two problems to contend with, a destabilized potential, and the current noise.

Something which was also seen without the Vycor tip was an overload in the current, when switching the current range; cr, to more sensitive current scales. The current overload indication light would light when the current read on the previous less sensitive scale was of a value which shouldn't come anywere near to causing overload at the next more sensitive scale. In order to gain insight into what was occurring here, and with the current noise and the potential instability, a comparison was made between the IBM and EG\&G potentiostats with varying supporting electrolyte concentrations, and different working electrodes; (w.e. s), to see the effects of these variables on the current noise and the potential instability (see Tables $1 \& 2$ ).

With the c.e. and the w.e. in the same compartment, from Table 1, which is for a Pt w.e., it is apparent that the best combination of instrument and supporting electrolyte solution, for solving the instability in the potential, the current noise problems, and for stepping down to as sensitive a current range as possible (without current overload), is the IBM potentiostat with $a \leq 0.1$ M TEATFB solution. Therefore, from here on this instrument was used as the potentiostat. This conclusion can be explained by looking at Table 1, from which it is apparent that 
Table 1 .

PLATINUN WORRING ELECTRODE

\begin{tabular}{|c|c|c|c|c|}
\hline $\begin{array}{l}\text { Solution composition } \\
\text { and concentration: }\end{array}$ & $\begin{array}{l}1.0 \mathrm{H} \\
\text { TEATFB }\end{array}$ & $\begin{array}{l}0.2 \mathrm{H} \\
\text { TEATFB }\end{array}$ & $\begin{array}{l}0.1 \mathrm{H} \\
\text { TEATFB }\end{array}$ & $\begin{array}{l}0.025 \mathrm{H} \\
\text { TEATFB }\end{array}$ \\
\hline $\begin{array}{l}\text { Current range where } \\
\text { IBM potentiostat lst } \\
\text { shows a jump in its } \\
\text { reading and the size } \\
\text { of the jump }\end{array}$ & $\begin{array}{l}1.0 \mathrm{uA} \\
\text { current } \\
\text { range } \\
\text { and } \\
5.4 \mathrm{UA} \\
\end{array}$ & $\begin{array}{l}1.0 \mathrm{uA} \\
\text { current } \\
\text { range } \\
\text { and } \\
5.4 \mathrm{uA} \\
\end{array}$ & $\begin{array}{l}1.0 \mathrm{uA} \\
\text { current } \\
\text { range } \\
\text { and } \\
3.4 \mathrm{UA} \\
\end{array}$ & $\begin{array}{l}1.0 \mathrm{uA} \\
\text { current } \\
\text { range } \\
\text { and } \\
430 \mathrm{nA} \\
\end{array}$ \\
\hline $\begin{array}{l}\text { Current range where } \\
\text { EGdr potentiostat } \\
\text { overloads }\end{array}$ & $10.0 \mathrm{uA}$ & $10.0 \mathrm{uA}$ & $10.0 \mathrm{uA}$ & $10.0 \mathrm{uA}$ \\
\hline $\begin{array}{c}\text { Current range where } \\
\text { IBM potentiostat } \\
\text { overloads }\end{array}$ & $1.0 \mathrm{uA}$ & $1.0 \mathrm{UA}$ & $0.2 \mathrm{UA}$ & $0.1 \mathrm{uA}$ \\
\hline $\begin{array}{l}\text { Size of oscillation } \\
\text { in potential at a } \\
\text { gain setting of } 2 \\
\text { and current range of } \\
10 \text { uA IBM }\end{array}$ & $\begin{array}{l}10 \text { mvs } \\
\text { peak } \\
\text { to } \\
\text { peak }\end{array}$ & $\begin{array}{l}10 \text { mvs } \\
\text { peak } \\
\text { to } \\
\text { peak }\end{array}$ & $\begin{array}{l}\text { mvs } \\
\text { peak } \\
\text { to } \\
\text { peak }\end{array}$ & $\begin{array}{l}8 \text { mvs } \\
\text { peak } \\
\text { to } \\
\text { peak }\end{array}$ \\
\hline $\begin{array}{l}\text { Size of oscillation } \\
\text { in potential at a } \\
\text { gain setting of } 2 \\
\text { and a current range } \\
\text { of } 10 \text { uA; EG\&G }\end{array}$ & couldn't & measure, & urrent & erload. \\
\hline $\begin{array}{l}\text { Size of oscillation } \\
\text { in potential at a } \\
\text { gain setting of } 1 \\
\text { and a current range } \\
\text { of } 2.0 \mathrm{UA} \text {; IBM }\end{array}$ & size of os & cillation & impercepti & bly small \\
\hline $\begin{array}{l}\text { Size of oscillation } \\
\text { in potential at a } \\
\text { gain setting of } 1 \\
\text { and a current range } \\
\text { of } 1.0 \text { UA; EG\&G }\end{array}$ & couldn't & measure, & current & rerload. \\
\hline $\begin{array}{l}\text { Size of oscillation } \\
\text { in potential at a } \\
\text { gain setting of } 1 \\
\text { and a current range } \\
\text { of } 1.0 \mathrm{UA} \text {; IBM } \\
\end{array}$ & couldn't & measure, & current & bverload \\
\hline $\begin{array}{l}\text { Peak to Peak current } \\
\text { noise EG\&G potentio- } \\
\text { stat } 100 \text { UA current } \\
\text { range }\end{array}$ & $4.0 \mathrm{uA}$ & $1.6 \mathrm{uA}$ & $1.6 \mathrm{uA}$ & $800 \mathrm{nA}$ \\
\hline $\begin{array}{ll}\text { Peak to Peak } & \frac{\mathrm{cr}}{00} \\
\text { current noise } & 100 \mathrm{~A} \\
\text { IBM potentio- } & 2.0 \mathrm{uA} \\
\text { stat. } & 0.2 \mathrm{uA}\end{array}$ & $50.0 \mathrm{nA}$ & $45.0 \mathrm{nA}$ & $\begin{array}{l}300.0 \mathrm{nA} \\
50.0 \mathrm{nA}\end{array}$ & $\begin{array}{r}300.0 \mathrm{nA} \\
50.0 \mathrm{nA} \\
40.0 \mathrm{nA} \\
\end{array}$ \\
\hline
\end{tabular}


Table 2 .

GRAPHITE WORRING ELECTRODE

\begin{tabular}{|c|c|c|c|c|}
\hline $\begin{array}{l}\text { Solution composition } \\
\text { and concentration: }\end{array}$ & $\begin{array}{l}1.0 \mathrm{H} \\
\text { TEATFB }\end{array}$ & $\begin{array}{l}0.2 \mathrm{M} \\
\text { TEATFB }\end{array}$ & $\begin{array}{l}0.1 \mathrm{H} \\
\text { TEATFB }\end{array}$ & $\begin{array}{l}0.025 \text { H } \\
\text { TEATFB }\end{array}$ \\
\hline $\begin{array}{l}\text { Current range where } \\
\text { IBM potentiostat lst } \\
\text { shows a jump in its } \\
\text { reading and the size } \\
\text { of the jump. }\end{array}$ & $\begin{array}{l}1.0 \mathrm{uA} \\
\text { current } \\
\text { range } \\
\text { and } \\
-3.5 \mathrm{AA} \\
\end{array}$ & $\begin{array}{l}1.0 \mathrm{uA} \\
\text { current } \\
\text { range } \\
\text { and } \\
-1.2 \mathrm{uA} \\
\end{array}$ & $\begin{array}{l}1.0 \mathrm{uA} \\
\text { current } \\
\text { range } \\
\text { and } \\
-0.3 \mathrm{uA} \\
\end{array}$ & $\begin{array}{l}\text { doesn't } \\
\text { jump }\end{array}$ \\
\hline $\begin{array}{l}\text { Current range where } \\
\text { EGSG potentiostat } \\
\text { overloads }\end{array}$ & $10.0 \mathrm{uA}$ & $10.0 \mathrm{uA}$ & $1.0 \mathrm{uA}$ & $1.0 \mathrm{uA}$ \\
\hline $\begin{array}{l}\text { Current range where } \\
\text { IBM potentiostat } \\
\text { overloads }\end{array}$ & $1.0 \mathrm{uA}$ & 0.1 UA & $0.1 \mathrm{uA}$ & doesn't \\
\hline $\begin{array}{l}\text { Size of oscillation } \\
\text { in potential at a } \\
\text { gain of } 2 \text { and a } \\
\text { current range of } 10 \\
\text { uA; IBM }\end{array}$ & $\begin{array}{l}60 \text { mvs } \\
\text { peak } \\
\text { to } \\
\text { peak }\end{array}$ & $\begin{array}{c}140 \text { mvs } \\
\text { peak } \\
\text { to } \\
\text { peak }\end{array}$ & $\begin{array}{l}180 \text { mvs } \\
\text { peak } \\
\text { ts } \\
\text { peak }\end{array}$ & $\begin{array}{l}120 \text { mvs } \\
\text { peak } \\
\text { to } \\
\text { peak }\end{array}$ \\
\hline $\begin{array}{l}\text { Size of oscillation } \\
\text { in potential at a } \\
\text { gain of } 2 \text { and a } \\
\text { current range of } 10 \\
\text { UA; EG\&G }\end{array}$ & couldn't & (2) & urrent & 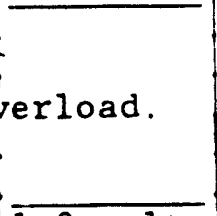 \\
\hline $\begin{array}{l}\text { Size of oscillation } \\
\text { in potential at a } \\
\text { gain of } 1 \text { and a } \\
\text { current range of } 2.0 \\
\text { UA; IBM }\end{array}$ & $\begin{array}{l}200 \text { mvs } \\
\text { peak } \\
\text { to } \\
\text { peak }\end{array}$ & $\begin{array}{l}600 \text { mvs } \\
\text { peak } \\
\text { to } \\
\text { peak }\end{array}$ & $\begin{array}{l}800 \text { mvs } \\
\text { peak } \\
\text { to } \\
\text { peak }\end{array}$ & $\begin{array}{l}1.2 \text { volts } \\
\text { peak } \\
\text { to } \\
\text { peak }\end{array}$ \\
\hline $\begin{array}{l}\text { Size of oscillation } \\
\text { in potential at a } \\
\text { gain of } 1 \text { and a } \\
\text { current range of } 1.0 \\
\text { uA; EG\&G }\end{array}$ & couldn't & measure, & urrent & rload. \\
\hline $\begin{array}{l}\text { Size of oscillation } \\
\text { in potential at a } \\
\text { gain of } 1 \text { and a } \\
\text { current range of } 1.0 \\
\text { UA; IBM }\end{array}$ & overload & $500 \mathrm{mv}$ & $800 \mathrm{mv}$ & 1.2 volts \\
\hline $\begin{array}{l}\text { Current noise EG\&G } \\
\text { potentiostat. } \\
100 \text { uA current } \\
\text { range }\end{array}$ & $1.2 \mathrm{UA}$ & $800 \mathrm{nA}$ & $700 \mathrm{nA}$ & \\
\hline $\begin{array}{ll}\text { Current noise } & \frac{\mathrm{cr}}{00} \mathrm{uA} \\
\text { IBM potentio- } & 100 \mathrm{uA} \\
\text { stat current } & 2.0 \mathrm{uA} \\
\text { range; }(\mathrm{cr}) & \end{array}$ & $\begin{array}{l}400 \mathrm{nA} \\
\text { not } \\
\text { stable }\end{array}$ & $\begin{array}{l}\text { UNSTABLE } \\
\text { see text }\end{array}$ & $\begin{array}{l}\text { UNSTABLE } \\
\text { see text }\end{array}$ & $\begin{array}{l}\text { UNSTABLE } \\
\text { see text }\end{array}$ \\
\hline
\end{tabular}


the IBM instrument allows much greater current sensitivity and shows smaller noise in the current. The graphite electrode didn't show all of these trends but this was later found to be because the design of the reference electrode and the graphite w.e. didn't permit as close a proximity between these electrodes as with the Pt electrode.

As with regards the noise in the current, the IBM potentiostat showed the smallest noise and the noise decreased with decreasing supporting electrolyte concentration, as well as when the current range was stepped to increasingly more sensitive scales. However, at the lower concentrations it becomes a concern as to whether or not the solution is sufficiently conducting.

With a new reference electrode design, permitting greater proximity between the graphite w.e. and the r.e.; approximately a $1 \mathrm{~mm}$ distance, a $0.1 \mathrm{M}$ TEATFB solution, the IBM potentiostat, and a sensitive enough current scale $(\leq 0.1 \mathrm{uA})$, the potential showed the most stability, current overload did not occur until well into the nanoamp scale, and the noise in the current was the smallest (disregarding the lower supporting electrolyte concentrations as a means of further decreasing the noise). The fact that decreasing the supporting electrolyte concentration decreased the amplitude of the current noise suggested that it was being picked up from somewhere in the $1 \mathrm{ab}$.

The computer is interfaced with the potentiostat through a cable which runs almost the length of the lab, (approximately 25 
feet). It was thought that this cable could be acting as an antenna but the fact that it was double shielded, with each shield grounded, made this unlikely. The computer was situated so far from the potentiostat in order to avoid accidents involvirg wet chemistry and the computer. To make sure that the cabling wasn't acting as an antenna, the potentiostat was temporarily brought over next to the computer along with the electrochemical cell and the cabling which connects the computer and the potentiostat was coiled up and further shielded. No change in the magnitude of the noise was seen. Therefore, it was decided that as long as a sensitive enough current range setting could be reached, (i.e. approximately $50 \mathrm{nA}$ ), the noise could be lived with.

\section{DEVELOPING A TEGHNIQUE}

In order to simplify the capacitance measurements the input waveform was simplified to a single TVS. This avoids problems which could arise due to a low ratio of point number:mv scanned for the TVS. The modes of Asystant+ Software now used for data collection were the "High Speed Recorder Mode" or "the Signal Averager Mode". These had to be used in order to collect data at a sufficiently high rate to resolve the charging/discharging curves. 
Each of these modes collects data for no more and no less than one second at a rate of $7500 \mathrm{pts} / \mathrm{sec}$. The "Signal Averager Mode" was found most convenient for collecting the data since it collected at one second intervals repeatedly, allowing you to choose which scan you wanted to save. Unfortunately, even using a $7500 \mathrm{pts} / \mathrm{sec}$ acquisition rate leaves only 75 points for resolving the output from a $100 \mathrm{~Hz}$ input signal. This is high enough resolution to measure the capacitance but requires a slightly different technique as will be described later.

However, neither of these two modes of Asystant+ are capable of simultaneously collecting data and generating a waveform. Therefore when collecting data using "the Signal Averager" the waveform had to be generated by the potentiostat.

\section{MEASURING THE CAPACITANCE}

Myers, Cowherd, and Steuernage1 (21), Gagnon and co-workers (22), and Oren and Soffer (16), all use a graphical method to measure the capacitance from the current output of the TVS input. Problems with a graphical solution arise in the presence of a distributad capacitance, a faradalc current, or slow charging sites. In the absence of any of these, the current output should look like that shown in figure 10a. For the output in figure 10a, the capacitance can be calculated from $C_{d}-1_{c} / \nu$ (where $i_{c}$ is 
shown in figure 10a). If a faradaic current is present, the current output should look like that shown in figure 10b; here the capacitance can be calculated from the equation $C-\left(i_{1}+i_{2}\right.$ $(2 \nu)$, where $1_{1}=1_{c}+1_{f}$ and $1_{2}-1_{c}-1_{f},\left(1_{f}\right.$ is the faradaic current), and therefore $i_{1}+1_{2}-21_{c}$; equation 1 , (see figure $10 \mathrm{~b}$ for an illustration of the quantities $i_{1}$ and $i_{2}$ ). Equation 1 is valid because the potential the instant after the point of the discontinuity in the TVS hasn't changed significantly and so any faradaic current should remain of approximately the same magnitude, while the capacitive charging current switches sign the instant the scan direction is reversed.

In the situations when there is a current $i_{c}$ due to a distributive capacitance with and without a faradaic current, the curront looks like that shown in figure $10 \mathrm{~d}$ and $c$, respectively. Here, a graphical solution is difficult. In this situation, when there is a distributive capacitance, possibly in the presence of a faradaic current, Gagnon and co-workers $(19,20,22,24)$ make use of a complex model, while Oren and Soffer (16) make due with a graphical solution. Soffer manages to deconvolute his charging/discharging curves using the following method (see figure 11): the point where the slope of the charging curve begins to fall off from its initial steep value, point a in figure 11 , is taken to be the end of the fast charging region and the absolute magnitude of the current difference between this point and that where the previous slow process ended, point b in figure 11 , 


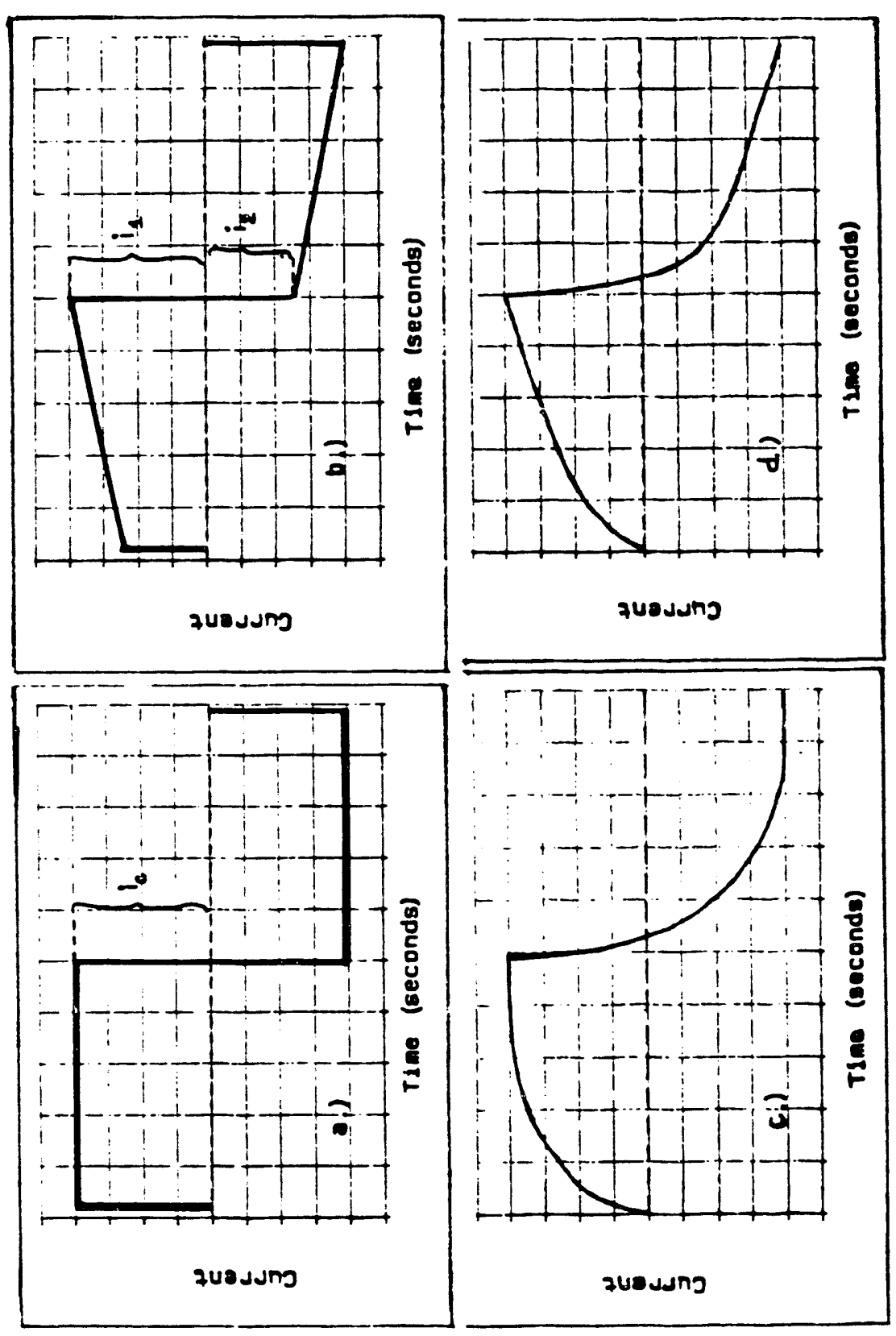

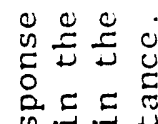

\& 000

늗

ه 口

4 v

5

U艺

อ車造

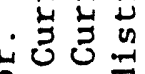

웅

ปิ

ํํㅇ

范

U

๙

ปั

บ

है

世豞

0

约 $\approx=\pi$

둥

崖出

की

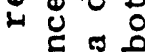

L

ह की 4

म

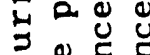

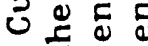

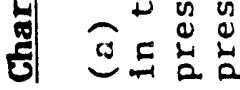

$\stackrel{1}{0}$ 


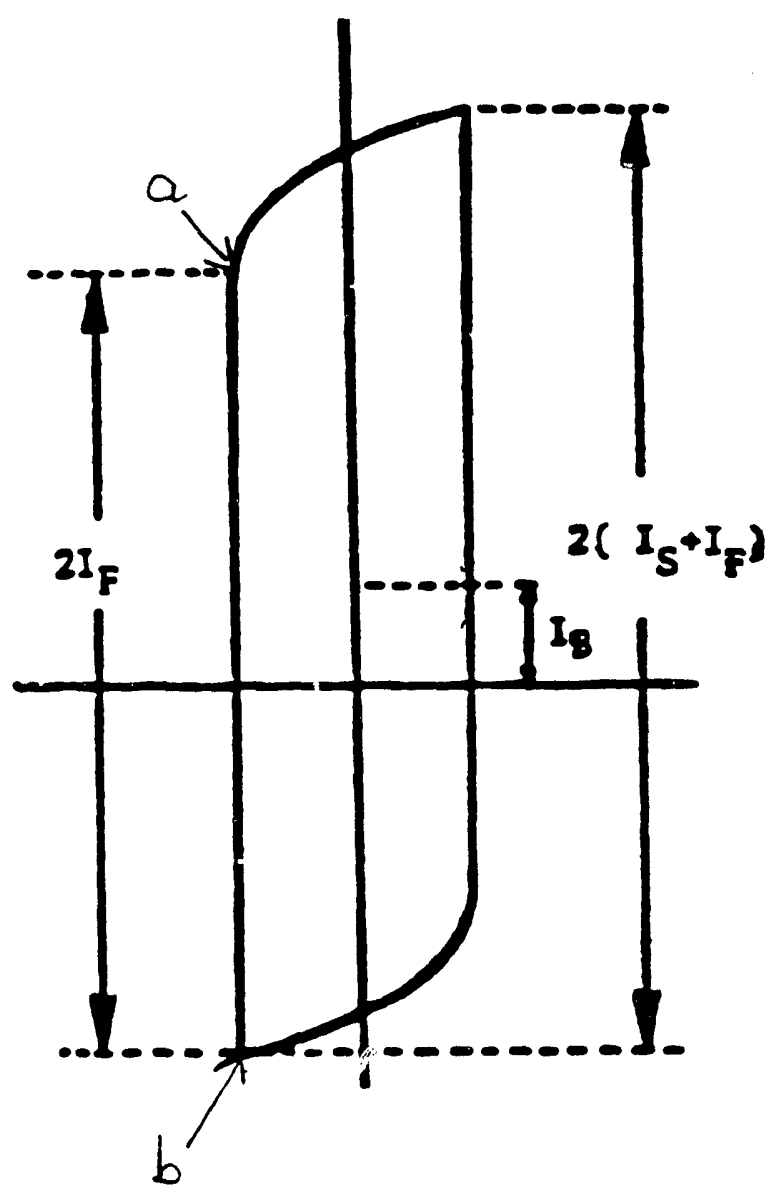

Figure 11. Soffer's Deconvolution

This figure shows what Soffer calls a differential voltammogram; in the language of this work it is a $c / d$ curve wrapped around itself. The $y$ axis is current and the $x$ axis is potential, as opposed to the independent variable used in this work; time. Soffer managed to deconvolute this graphically, as described in this thesis. Reprinted with permission from Oren, Y.; Soffer, A. J. Electroanal. Chem. 1985, 186, p 68. Copyright (1985), Elsevier Science Publishing Co. 
(this point corresponding to the discontinuity in the TVS), is taken to be two times the fast charging current. The absolute difference in the current values at the two discontinuities in the current curve is taken to be equal to 2 times $\left(i_{s}+i_{f}\right) i_{s}$ representing the slow charging current and $i_{f}$ representing the fast charging current.

Soffer's $(14,16)$ charging/discharging curves, which he calls differential voltammograms, are not centered around zero current. The amount of current by which his differential voltammograms are off centered from zero, he assigns to $i_{b}$; the background current. The charging/discharging curves, c/d curves, for an ideal capacitor are centered around zero current (see figure $7 b$ ).

\section{RESULTS FROM THIS MORR}

The capacitive charging current increases proportionally to the scan rate and consequently, the signal to noise ratio for the current output does also. The output, shown in figure 12, resulting from a $50 \mathrm{mv}$ amplitude, $4 \mathrm{~Hz}$, TVS; equivalent scan rate of $400 \mathrm{mv} / \mathrm{sec}$, would therefore be expected to have a relatively high signal to noise ratio. This figure shows a complete charging/discharging curve. From this, it is clear that locating the relevant points even with data collected at this high a scan rate is impossible with the existing level of noise. 

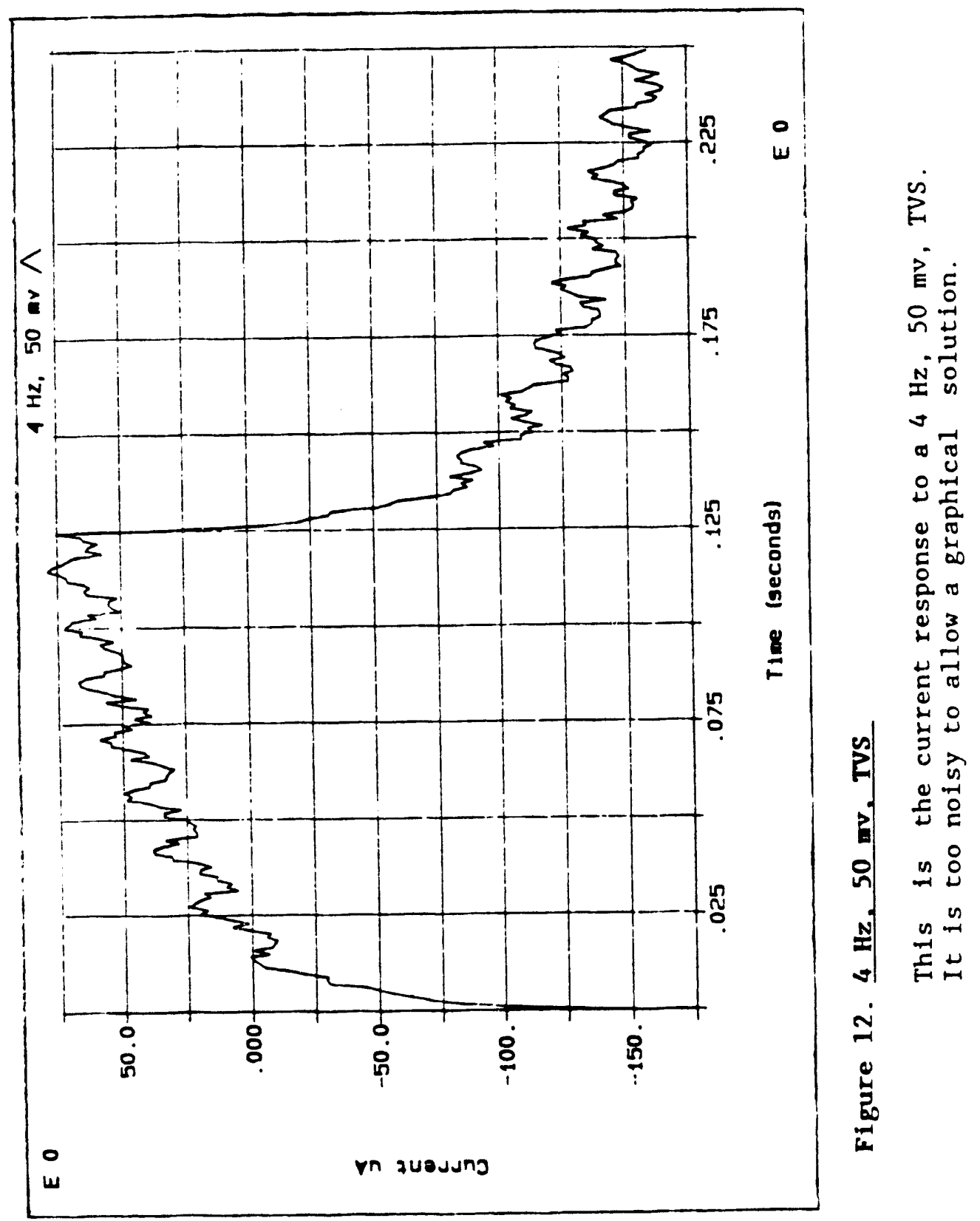
It was unclear what form the $c / d$ curves obtained so far were in. Was the apparent slow charging process seen in figure 12, a distributive capacitance, a faradaic current, or a combination of the two? In any case, it wasn't possible to measure the capacitance using a graphical method because of the low signal to noise ratio and the presence of a slow process, (possibly a distributive capacitance). So how was the capacitance going to be measured?

Since further reduction in the noise was unlikely, an attempt was thus made to reduce the amplitude of the input waveform, (which results in a decrease in the equivalent scan rate), with the hope that this might eliminate or reduce the slow process. However, another problem existed. The IBM potentiostat will not scan a TVS with an amplitude smaller than $30 \mathrm{mv}$. Since the differential capacitance; $C_{d}$, is a function of potential, its value could be changing during a $30 \mathrm{mv}$ sweep. This large a sweep would allow less time for the establishment of a stable, in the words of oren and Soffer, "time independent capacitance" measurement (16). Also according to oren and Soffer, "when slowly charging sites are excited throughout a wide potential range, the momentary electrode potential may be at $V_{m}$, while the charging current may contain contributions of sites that should have been relaxed at potentials remote from $v_{m} \cdot(16, p .65)$. Soffer's solution to this problem is to slow down the sweep rate but, as he points out, this is limited by how small a current can 
be measured with available instrumentation and in the presence of existing noise.

Another possibility, not mentioned by Soffer is to minimize the sweep amplitude, so that more time is spent within a given potential range. Soffer uses a $50 \mathrm{mv}$ sweep amplitude. This seems large, especially when considering that $C_{d}$ is the instantaneous charging current divided by the scan rate; $i_{c} / d v / d t$, which according to Delahay (23): "can be measured provided the excursion of potential $\partial E$ in measurements does not exceed a few millivolts." In light of this statement, and also that made by Soffer, regarding the slow charging sites, it seemed important to not only minimize the sweep rate but also the sweep amplitude, and much below 30 or $50 \mathrm{mv}$.

Because the IBM potentiostat can't sweep a range smaller. than $30 \mathrm{mv}$, an instrument which could do this was found. This was a Dana Exact Waveform Generator model \#120. When a voltage divider (total input impedance $100 \mathrm{k} \Omega$ ) was imposed across the output from this, a sweep amplitude of a few mvs was attained. This function generator has a frequency range from $1 \mathrm{~Hz}$ to $3 \mathrm{MHz}$, which, with the $3 \mathrm{mv}$ amplitude signal, yields an equivalent scan rate range of $6 \mathrm{mv} / \mathrm{sec}$ to $18 \mathrm{kV} / \mathrm{sec}$. The data which a $1 \mathrm{~Hz}, 6 \mathrm{mv}$ amplitude TVS yields is shown in figure 13. A slow charging process is still present and a graphical solution is difficult because determination of the point in the charging curve where the fast charging process ends and the slow one begins is still 

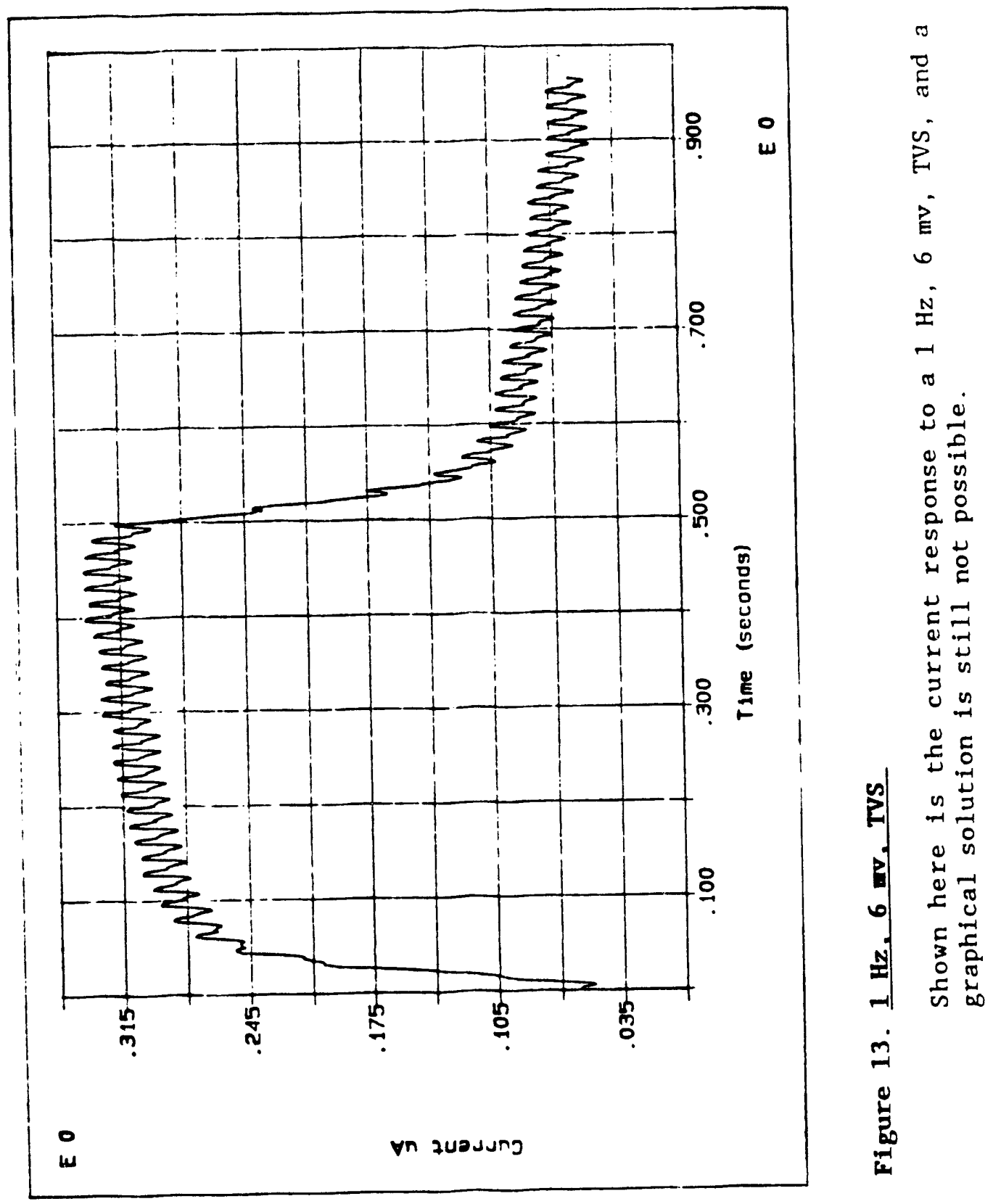
very subjective. Also in this figure, a large background current is apparent from the fact that the entire $c / d$ curve lies above zero of current.

\section{FARADAIC CURREAT}

If the slow charging process isn't a charging process at all but is actually a faradaic current, it is believed that the most likely contaminant is water. Water could be introduced from the electrode, the supporting electrolyte, or the solvent. The electrode is polished in a dp slurry made in water, TEATFB is hygroscopic, and acetonitrile is usually contaminated with water and is difficult to purify. However, the acetonitrile is distilled over $\mathrm{CaH}_{2}$, and the time to which the TEATFB is exposed to open air is minimized, so it was thought that the electrode was the most likely source. In order to see if this was the case, graphite electrodes received Vacuum Heat Treatment; VHT, (see the experimental section for details concerning this procedure). This produced a higher background current and an increase in the magnitude and apparent linearity of the slow process. These results suggested that water was not the cause, at least not water from the electrode. Further details about the VHT results will be discussed later on.

When a platinum electrode was used as the working electrode, a slow process was also seen. Whether this had the 
same cause as the slow process on graphite was unknown but if it had, it seems to support the idea that a faradaic current is involved.

This exhausted all avenues for changing the quality of the data and it thereby became apparent that the charging curve was incorrigible and would have to be lived with in its present form. Therefore, an attempt was made to fit a function to the data.

\section{FITTING THE DATA:}

First, a function was needed. No function for the charging of the edl with a TVS could be found in any textbook, nor could one for charging a parallel plate capacitor with a TVS be found in an electronics book. An equation which seemed to be applicable was seen in Gagnon's paper (24), however, it was unclear whether or not this was it. Therefore, an attempt was made to derive a function, this is shown in appendix 5. In this derivation, an equivalent circuit modeling the electrode-electrolyte interface is assumed and this is simplified down to a resistor and a capacitor in series. The resistance is that of the electrolyte solution and may include the resistance involved with the ions 
entering the internal structure and the capacitance is representative of the edl capacitance. With this circuit, a TVS input is imposed across 1 t. The resulting function is:

$$
1_{t}=\nu C\left(1-e^{-t / R C}\right) \quad \text { equation } 2
$$

; where $\nu$ is the scan rate in volts/sec, $1_{t}$ is the instantaneous current flowing through the circuit measured in amps, $C$ is the capacitance measured in farads, and $R$ is the resistance measured in ohms. As a consequence of the fact that the charging/dis charging curves are seldom centered around zero, as would be expected for an ideal capacitor, another term was added to the equation to simply offset the resulting function:

$$
i_{t}=\nu C\left(1-e^{-t / R C}\right)+a \quad \text { equation } 3
$$

The best fit achieved using Asystant+'s Curve Fitting Software is shown in figure 14. It was apparent from this that a single exponential term wasn't capable of fitting the charging curve. Therefore an attempt was made to fit with the sum of two exponential terms, using Asystant+'s Curve Fitting Software. The function was:

$$
i_{t}-\nu C_{1}\left(1-e^{-t / R_{1} C_{1}}\right)+a_{1}+\nu C_{2}\left(1-e^{-t / R_{2} C_{2}}\right)+a_{2}
$$

equation 4 

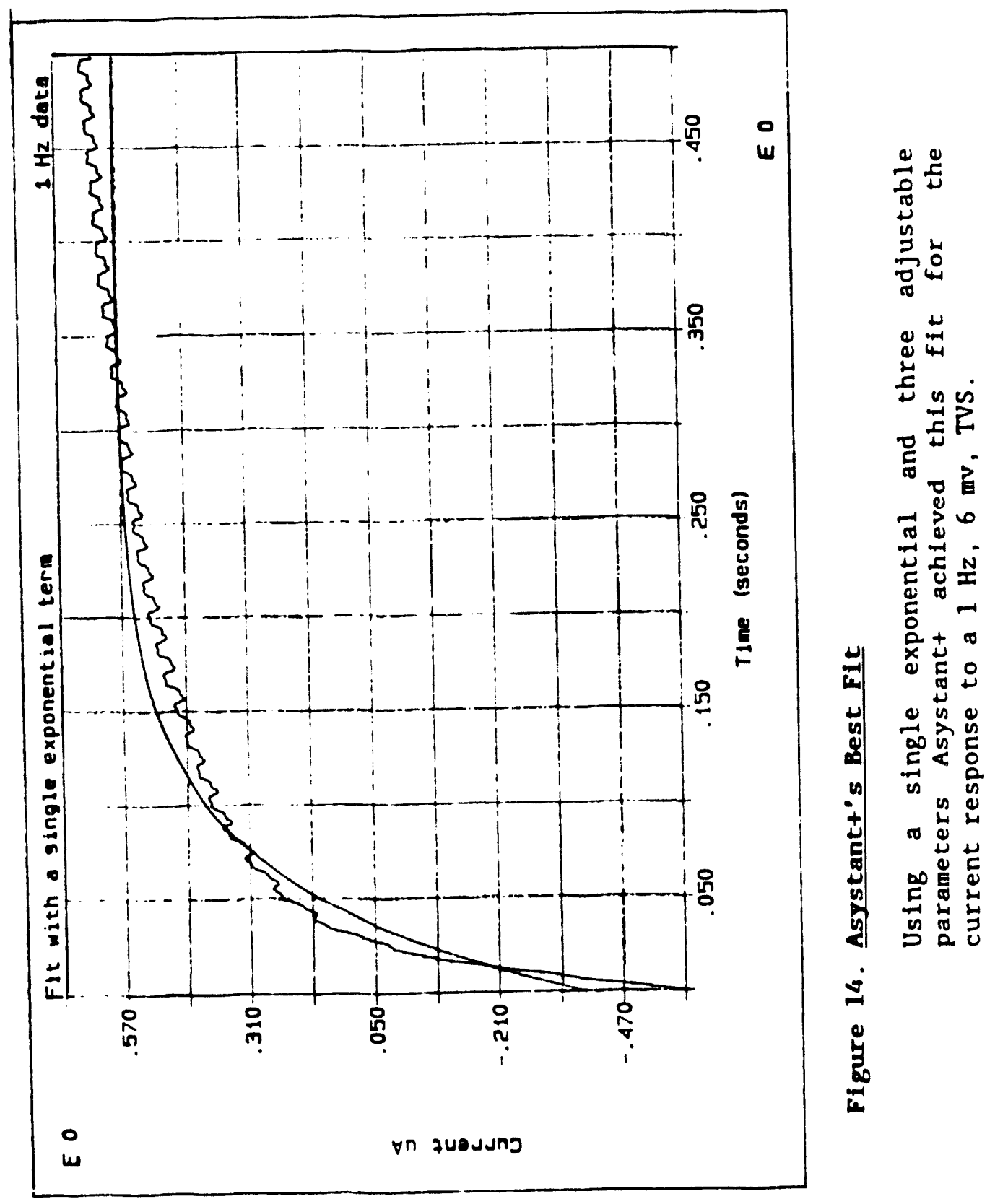
However, this exceeded the number of adjustable parameters this sof tware could manage.

Fortunately, my research advisor had recently finished writing a curve fitting program, in Fortran, for another application in our research group and with a few changes, this program turned out to be ideal for fltting the charging curves. A function is fit to the data by a modified simplex iteration procedure, minimizing the error defined as:

$\operatorname{error}=\sum_{1}^{\text {number of points }}$ [experimental $i_{t}-$ calculated $i_{t}$ ]
equation 5

Once altered for fitting charging curves, this program used equation 3 as the repeating term in a series of like terms, each with its own set of parameters for $R, C$, and $a$. How many of the terms were used was up to the user's discretion, but the program was written to accommodate a maximum of 15 adjustable parameters, with a minimum of three; since there are three parameters per term, this corresponds to a maximum of 5 terms, (see appendix 6 fcr a copy of this program).

The result for a single exponential fit using the simplex program was much the same as the result for a single exponential fit using Asystant,, shown in figure 14, but for a double exponential fit using the Simplex program, with two terms, like in equation 4, the fit is excellent (see figure 15). The dashed curve and the curve labelled with $S(s)$ are the separate 


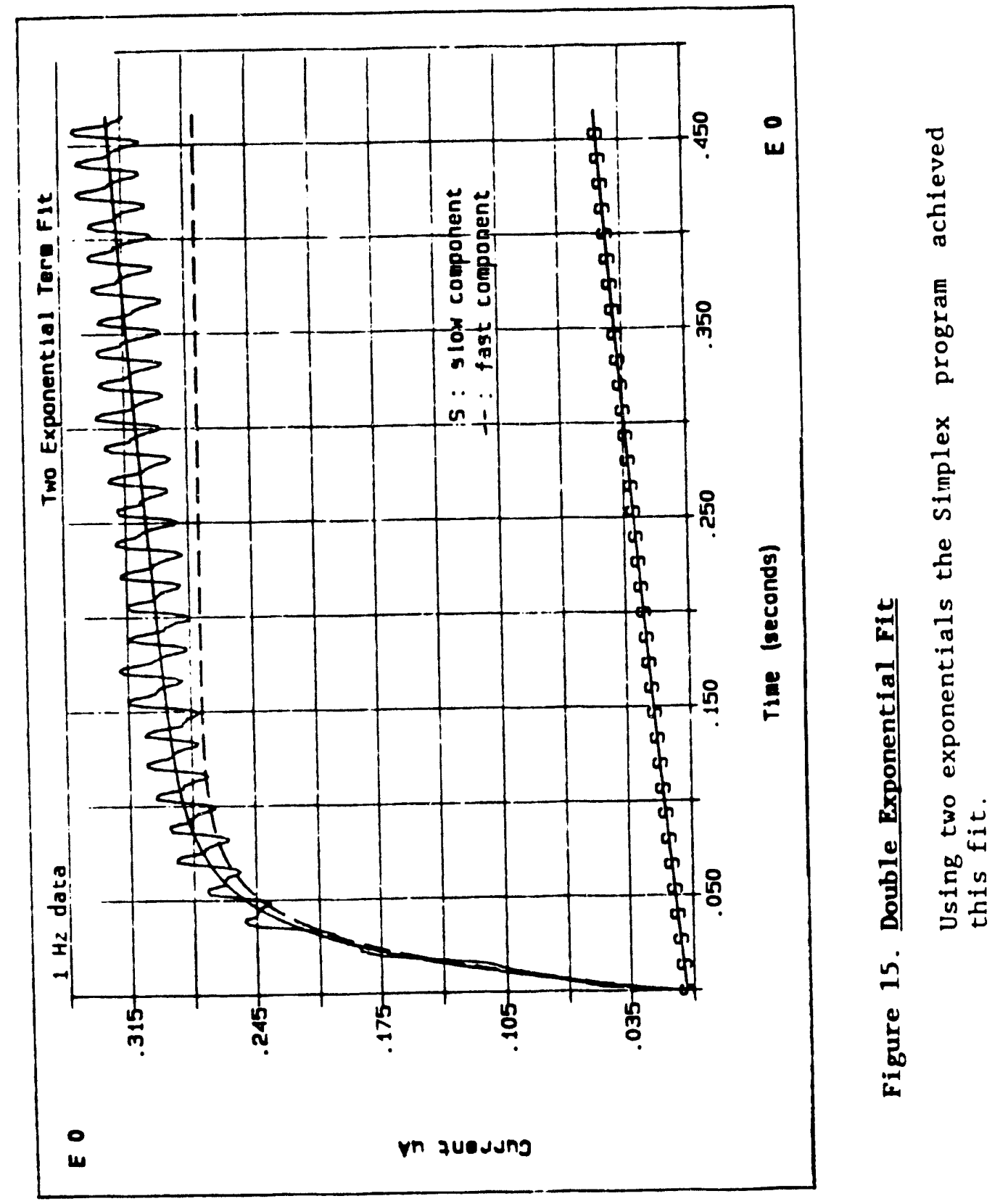


components making up the complete fic, which passes directly through the experimental data; the noisy curve. The parameters which the computer used to achieve this two term fit resulted in a slow and a fast charging capacitance; the rate of charging determined by the associated resistances. Thus, a method of measuring the capacitance had been developed.

\section{QUESTIONS ABOUT THE FIT}

However, some questions about the fit remained. Some good examples of the ki dd of fit which would incite these questions are shown in figure $16 a$ and $b$. Figure $16 a$ shows the separate exponentials used to fit the experimental charging curve, labelled with $S(s)$ and $F(s)$ for the fast and slow processes, respectively, and additionally shows the sum total of the two exponentials which passes directly through the experimental data. The experimental data can be identified by its noise. The separate exponenials lie far above and below the actual charging curve, yet their sum fits the experimental data flawlessly, and the computer program finds a minimum in the error at the point corresponding to this set of parameters. So, is this the right fit? Is it a unique fit?

Another related problem is if the charging and discharging curves for a single voltage sweep are each fit separately, (the program was only equipped to fit these separately at this time), 

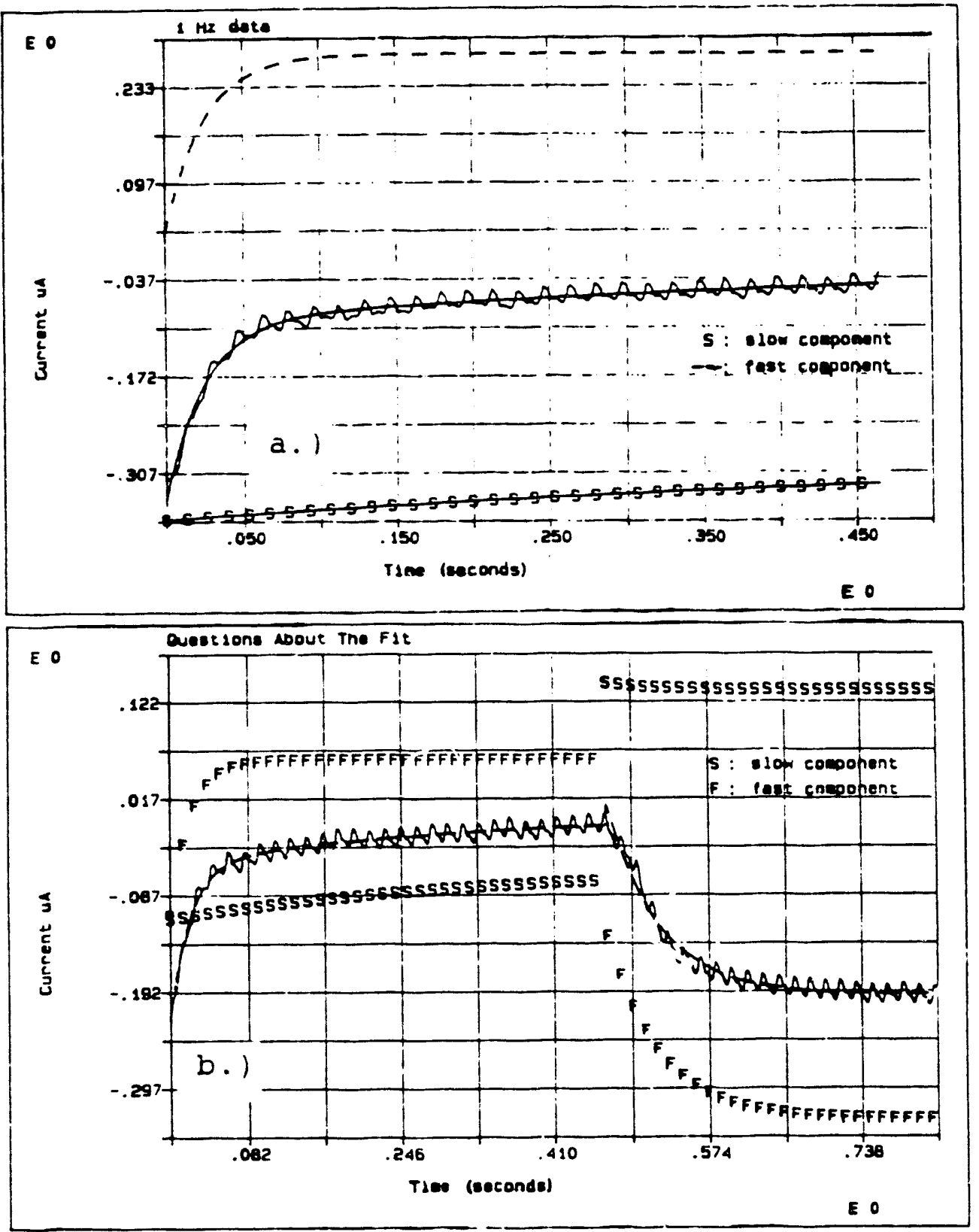

Figure 16. Questions about the Fit

(a) This figure is an example of the kind of fit that would incite questions about the validity, and uniqueness of the fit. (b) Should the separate components of the two halves of the curve connect with their corresponding other halves? 
and the two halves are then put back together using Asystant+ data manipulation, the slow and fast sections don't connect up to their corresponding other halves. In some instances, the two halves don't come even close to one another, see figure 16b. In figure $16 \mathrm{~b}$ are shown the experimental data, (again identifiable by the noise present in $1 t)$, the sum of the two components passing through the experimental data, and the individual components are the other curves. The slow component is labelled with $S(s)$ and the fast component is labelled with $F(s)$. Should these two halves connect and if so, why don't they?

In order to investigate the questions about the correctness and uniqueness of the fit, a minimum was found using the Simplex proglam for fitting the charging curve shown in figure 16a. From this fit, which used a function like equation 4 , the parameters $a_{1}$ and $a_{2}$ were added together. Half their sum was then given to each of a new $a_{1}$ and $a_{2}$, shifting the actual fitted components closer to each other and to the experimental data. A new minimum was then found starting with the new and equal $a_{1}$ and $a_{2}$, and otherwise the previous fitted parameter values. This minimum had the same values of $R_{1}, C_{1}, R_{2}$, and $C_{2}$ as the previous fit but now approximately equal values for $a_{1}$ and $a_{2}$. In the sense that the individual values of $a_{1}$ and $a_{2}$ are the only parameter values which change, this is a unique solution.

This is shown to be mathematically trivial if the parameters used to fit the data before $a_{1}$, and $a_{2}$ were summed, divided 
evenly, and then refit, are substituted into equation 4 and the fitted parameters used to fit the data after $a_{1}$ and $a_{2}$ were summed, divided evenly, and then refit are likewise substituted into another equation 4 and these two equations are then set equal to one another. Since $R_{1}, C_{1}, R_{2}$, and $C_{2}$ don't change with refitting the data, the only difference is in $a_{1}$ and $a_{2}$, the sum of which hasn't changed. The equality is therefore obvious; an infinite number of solutions are possible all with the same values of $R_{1}, C_{1}, R_{2}$, and $C_{2}$, with different individual values of $a_{1}$ and $a_{2}$, but the same sum of $a_{1}$ and $a_{2}$. Since the individual values of $a_{\text {a }}$ and $a_{2}$ are the only parameter values which change, this is a unique solution.

The answer to the second question of why the two halves don't match up, is the same as the answer just given, except a related question is interesting. Is it possible to fit both the charging and discharging halves of the curve with the same set of parameters? The answer to this question turns out to be yes, see figure 17. For this fit, the simplex program was altered so that it could attempt to fit both halves of the $c / d$ curve simultaneously and with the same set of parameters. Here, again the experimental data is identifiable as the noisy one and the curve which passes through it is the sum of the two components labelled $F$ for fast and $S$ for slow. This implies that the charging/discharging process seen here is reversible. 


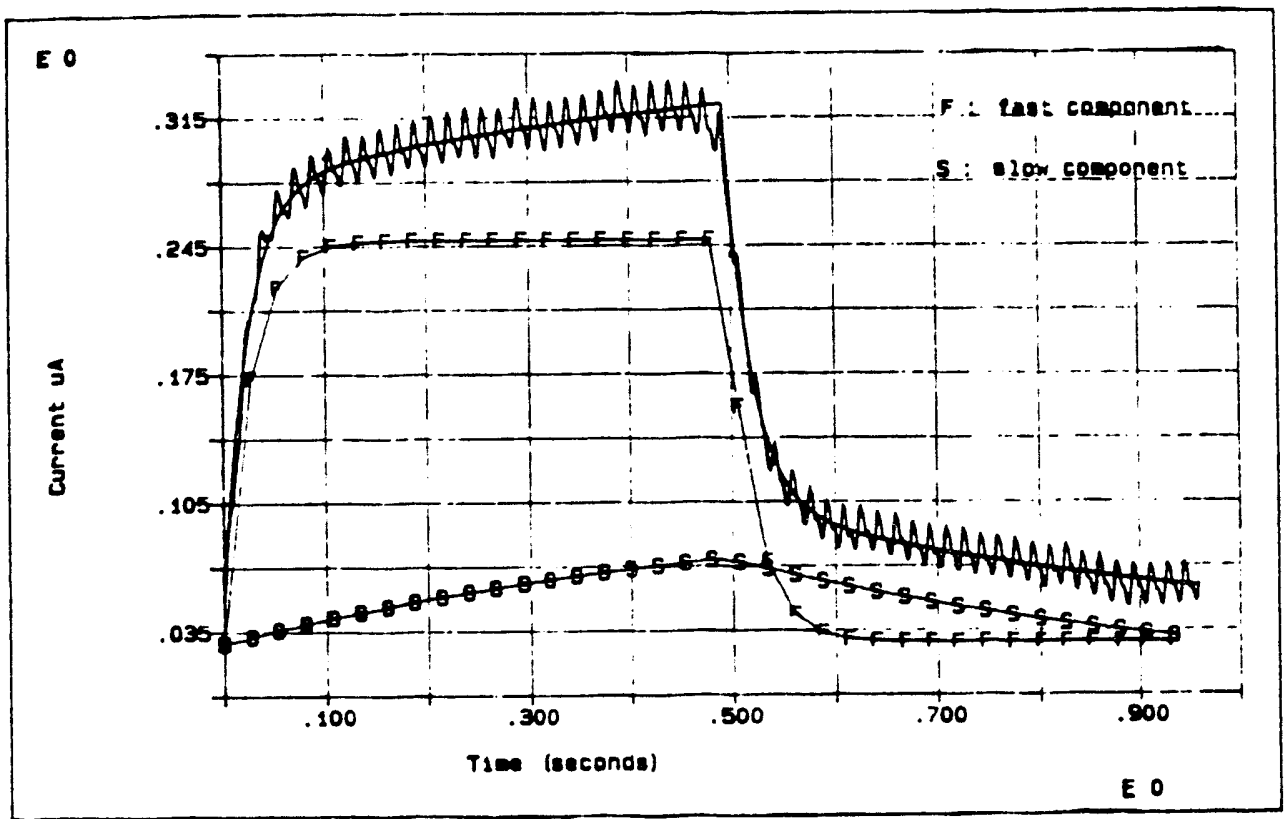

Figure 17. Fitting the whole $C / D$ Curve simultaneously

A single set of parameters can be used to fit the entire $c / d$ curve together. 


\section{FITTING INCLUDING FARADAIC CURREAT TBRH}

To try and once and for all eliminate a faradaic current as a possibility, the repeating unit of the function which the Simplex program uses to fit the data; equation 3, was altered to include a term proportional to the scan rate; $\nu$, multiplied by the time; $t$, and multiplied by an adjustable parameter; $d$. This product, $\nu$ dt, was merely added to the function. This was done since according to Gagnon (22), a faradaic current resulting from a TVS input gives rise to a response linear with time.

This function was used with one and two repetitions. When using two repetitions, one of the two d parameters was deliberately made very close to zero so that the resulting function had essentially only one of these terms. This was found to yield a closely approximating fit with one exponential but in order to produce the same flawless fit as seen earlier, two exponentials were needed. In figure 18a, the fit with one exponential is shown, in $18 \mathrm{~b}$ that with two exponentials, $18 \mathrm{c}$ with one exponential and one faradaic term, and in 18d the fit is with two exponentials and one faradaic term. From these figures, it appears that one exponential and one faradaic term don't fit the data as well as two exponentials with and without a faradaic term. The faradaic term is therefore not necessary but this does 

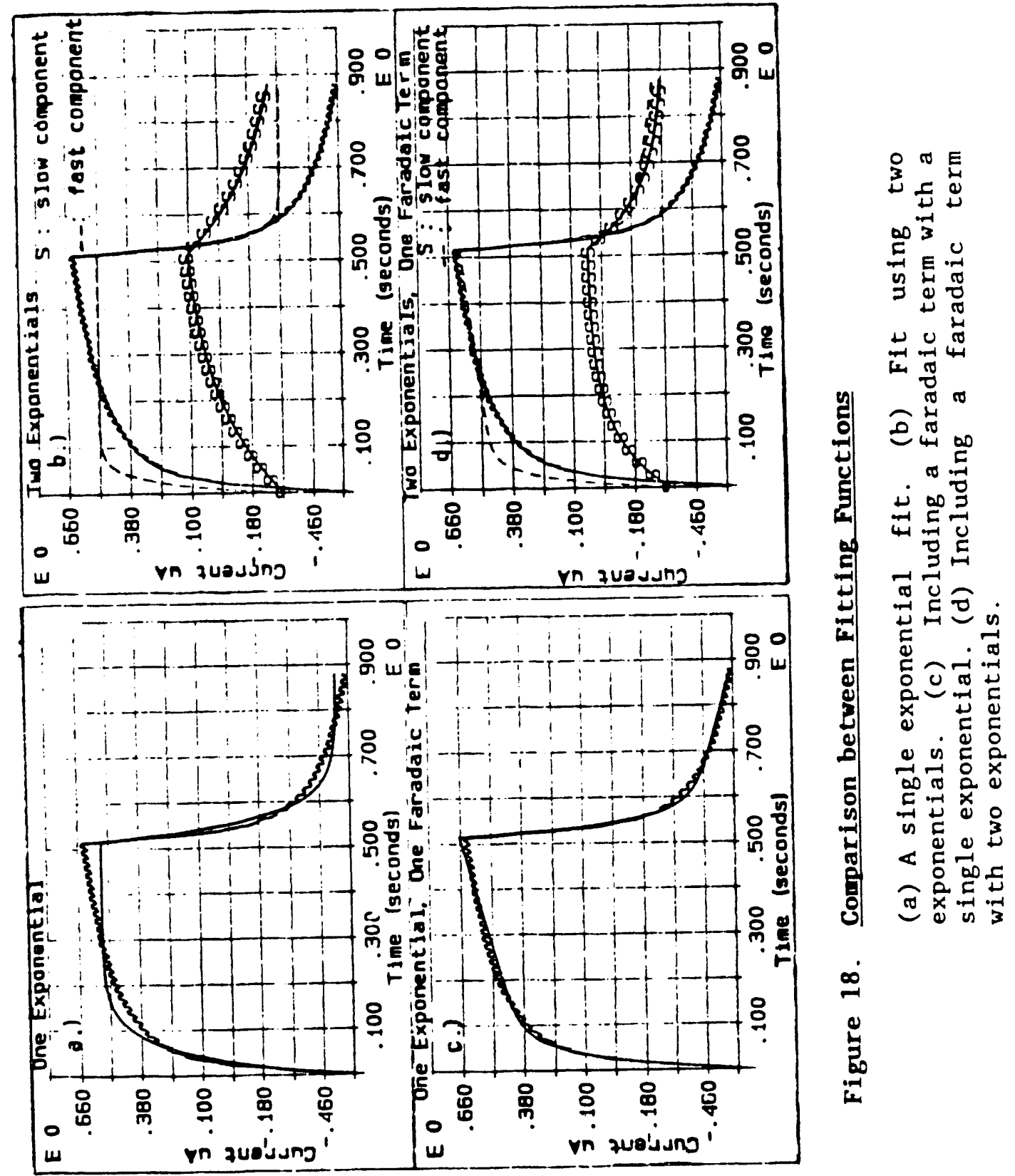
not rule out the possibility that a faradaic current may be present.

\section{ASSUIE NO FARADAIC CURRENT}

However, the fact that no peaks are visible in the cyclic voltammogram at the potentials where the $\mathrm{c} / \mathrm{d}$ curves are recorded, (see appendices $1 \& 2$ ), and that a faradaic term isn't required to fit the experimental data, seem a reasonable basis for making the tentative assumption that no faradaic current is present. Whether or not this is valid remains to be seen. Based on this, it was decided that the data would be fit with just the two exponentials, proceeding with the assumption that no faradaic current is present, and the two capacitance values obtained with the fit would be accepted at face value. If this assumption is incorrect, then something would probably reveal this. It was now time to obtain capacitance-potential curves; C-E curves.

V. RESULTS AND DISCUSSION:

GAPACITANCE VS POTENTIAL CURVES 
As made clear in appendices 1 and 2, a safe potential range, free from any apparent faradaic current, is $-0.500 \mathrm{~V}$ to $0.500 \mathrm{~V}$ vs the $\mathrm{Ag} / \mathrm{Ag}^{+}$electrode. This is the potential range in which the c/d curves were recorded.

None of the recorded $c / d$ curves are for the first TVS to which the electrode was exposed, at a particular potential. Rather, they are the recorded current response after any number of TVS(s). Therefore, for all the curves fitted in this report, the curves are starting from a charged state opposite in sign to the charging caused by the immediate voltage sweep, and as a result the initial current isn't zero. Since the charge stored at this starting state, for a small enough sweep, should ideally be equal and opposite to that at the end of the present voltage sweep, the $C_{d}$ values obtained from the curve fitting are believed to be twice that of the actual capacitance. When this hypothesis was tested by editing the data to, as best as possible, include only the charging from an uncharged state, it was found to be valid for the fast charging capacitance. Checking this for the slow process would be much more difficult, if not impossible, and therefore wasn't tried.

It was initially decided that both halves of the charging discharging curve would be fit simultaneously with a single set of parameters, since this would be like two averaged measurements in one. This was later found to have been a bac choice because when both halves are fit simultaneously, the boundary point or 
more accurately the index of the boundary point, where the scan direction is reversed, (which the modified simplex program required as input in order to fit both halves of the $c / d$ curve), was difficult to find exactly and fitting in this way zequired an order of magnitude more iterations and effort. Unfortunately, when this was realized some of the data in the present set hid already been fit in this way. Actually, the values obtained from these two different fitting methods are very similar, see figure 19. In this figure, the slow "capacitance"; $C_{s}$, is plotted as a function of potential for a $1 \mathrm{~Hz}, 5 \mathrm{mv}$ input TVS. Symbol B is the slow "capacitance" obtained from fitting using both curves and $H$ is that from fitting with only the charging half of the curve. Other parameters fit with these two methods showed similar consistency between the two methods and so data fit from here on, was fit using only the charging half of the $c / d$ curve.

\section{REPRODUCIBLE C-E CURVES}

The first point of interest was to check the reproducibility of the C.E curves. In figure 20 are shown the fast and slow capacitance vs potential curves for two sets of electrodes. Unless specified otherwise, these electrodes and all electrodes here after used, received only polishing and drying in the oven at $90 \mathrm{C}$, as pretreatment procedures. In figure 20 the data of one electrode from a set is represented with upper case letters and 


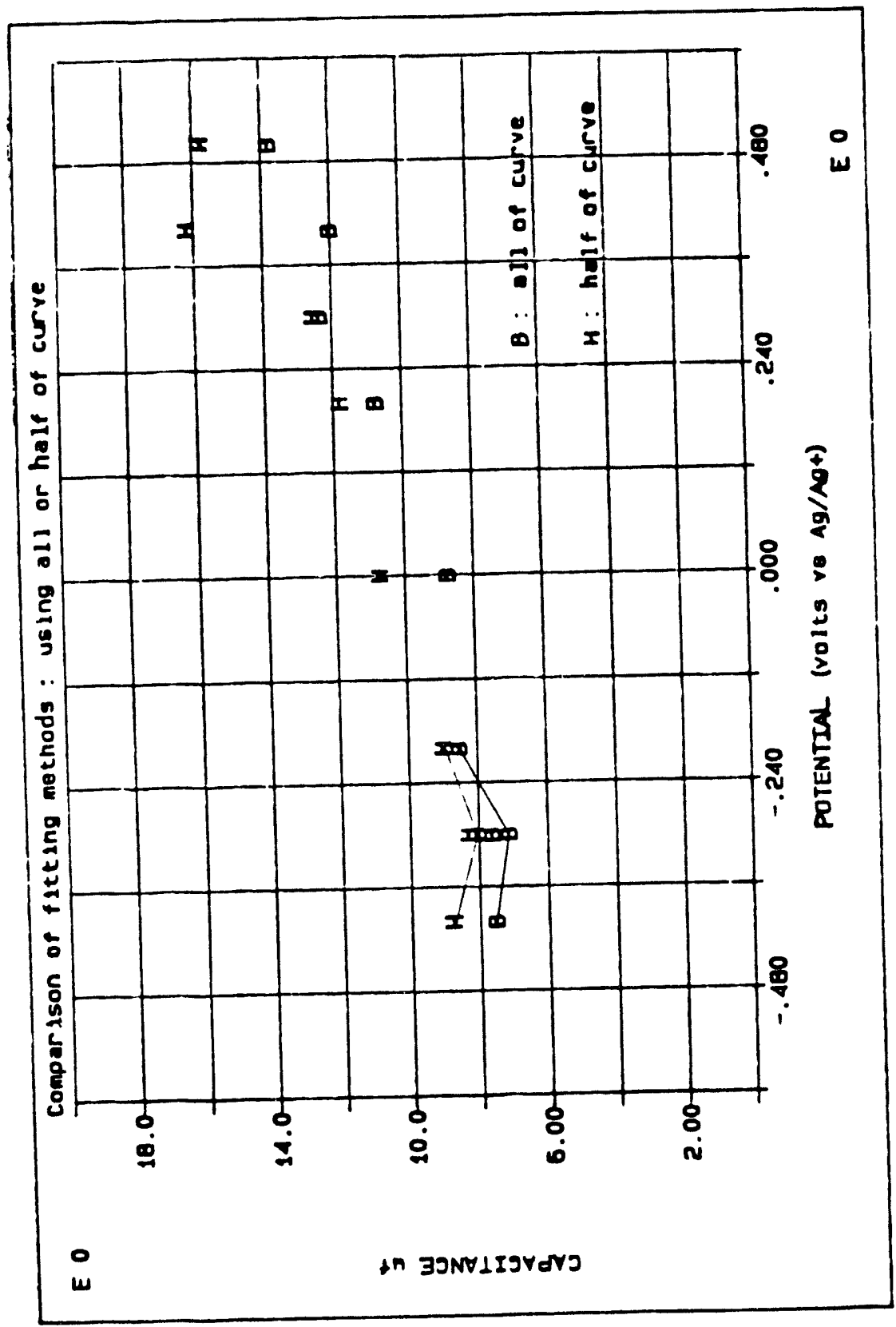

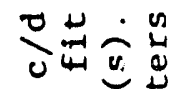
๑电岕 ذ志苋 $4 \overbrace{0}^{\pi} \frac{\pi}{3}$ 山U⿺辶寸

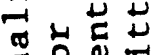
$\varepsilon$ 인 范苔总 考 马: .

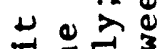

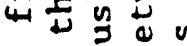
n 0 :

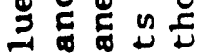
$\gg$. 出 昌 טणु

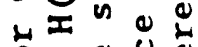
원 $0 \pm$ ¿ 30 5 ป ن 造类 क品出 (1) 0 少 H 50 . \% ปै …

2 n

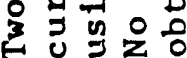

${ }_{0}^{0}$ 


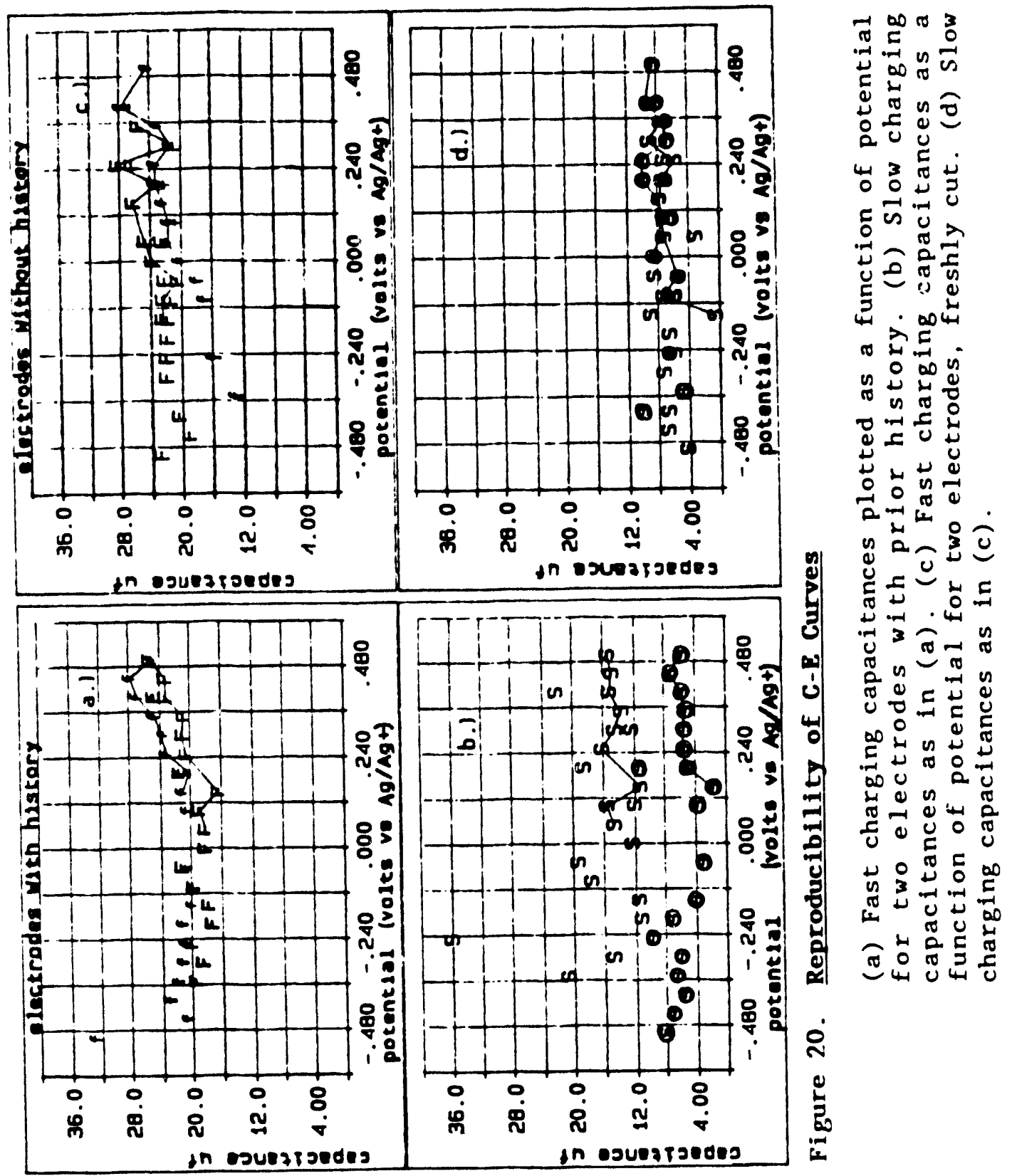


that of the other is represented with lower case letters. One set is presented in $a$ and $b$, on the left side of the figure, and the other set is presented in $c$ and $d$, on the right side of the figure. The letters $F$ or f represent the fast charging capacitance; $C_{f}$, of the corresponding electrodes and the $S$ and circled $s$, represent the slow charging capacitances of the corresponding electrodes. In these figures the connected points are the initial anodic excursion, starting at the left most point, which was the Open Circuit Potential; OCP.

$O C P$ is the potential measured immediately after electrode imnersion, and before any potentials are applied. OCP is measured during and immediately after $\mathrm{N}_{2}(\mathrm{~g})$ purging of the system and the most stable value is recorded. In all the C-E curves shown in this thesis, the plots start at OCP and, depending on the value of OCP, $50 \mathrm{mv}$ steps are taken away from OCP, in either the anodic or cathodic directions. OCP was found to be dependent on the electrode pretreatment procedure used, see appendix 4. If the OCP value is $>-0.100 \mathrm{~V}$ vs $\mathrm{Ag} / \mathrm{Ag}+$, then $50 \mathrm{mv}$ steps are made initially in the anodic direction, recording $c / d$ curves after each step. If the OCP is $\leq-0.100 \mathrm{vs} \mathrm{Ag} / \mathrm{Ag}+$, then $50 \mathrm{mv}$ steps are made in the cathodic direction. In either case, the initial step direction is reversed at one of the switching potentials; \pm 0.500 $\mathrm{V} v \mathrm{Ag} / \mathrm{Ag}+$, and then $100 \mathrm{mv}$ steps are taken in this new direction until what was $O C P$ is reached again. Here, the step direction is maintained but now in 50 mv steps until the other 
switching potential is reached; elther $\pm 0.500 \mathrm{~V}$. When both the anodic and cathodic limits have been reached in this manner, the experiment is finished. After every step, $100 \mathrm{mv}$, or $50 \mathrm{mv}$, a c/d curve is recorded.

The two electrodes used for figure $20 a$ and $b$ had previous histories of having been taken outside of the safe potential range. These electrodes showed poor reproducibility for the $C_{s}$ curves as is apparent in figure 20b. One set being on the order of a factor of three greater than the other.

In figure $20 \mathrm{c}$ and $d$ are shown the $C_{f}-E$, and $C_{s}-E$, curves for a different set of two electrodes. These electrodes were freshly cut for this experiment. The same symbol scheme is used here. The slow charging capacitances showed much greater reproducibility for these electrodes. The $C_{f}-E$ curves shown in figures $20 a$, and $c$, all showed very consistent values. The average value was on the order of $20-22$ uf.

Each point in figure 20 is a separately collected c/d curve, collected using computer data acquisition. The input wave had an amplitude of $5 \mathrm{mv}$ and the frequency was $1 \mathrm{~Hz}$. The experimental data from each series of recorded $c / d$ curves was then editted to include one and only one $c / d$ curve and the boundary index, where sweep direction was reversed, was located and recorded. This data was then convertad from Asystantt's character set to ASCII characters and up: aded to a VAX 3100 (model 38) computer. Here, the data was fit using starting parameter values from a previous fit 
involving similar data. This required an average of 10,000 iterations.

If the slow process is due to charging in between the microfissures, the disparity between the values of $C_{s}$ for different electrodes with history, seen in figure $20 \mathrm{~b}$, could be explained by one of them having been exposed to intercalation potentials which is known to increase the interlayer distance; (see Table 3). However, this experiment needs to be repeated many more times to substantiate this hypothesis.

\section{BACRGROUND CURRENT}

The c/d curves, which should have been centered at zero, were displaced sometiwes above and other times below zero by a biekground current. This was most apparent for the $1 \mathrm{~Hz}$ data and as the frequency of the TVS increased the magnitude of the current output signal for the fast process increased significantly, making the lisplacement from centering around zero both insignificant and imperceptible. Unlike Soffer and coworkers (14-18), who as dentioned earlier, also saw a background current in their experiments, we did not measure our background current from the displacement of the $c / d$ curve with resp-t to the zero of current. Instead, it was measured directly off of the nanoammeter of the potentiostat. Fint the $c / d$ curve was recorded at a given potential and then. after disconnecting the TVS input, 
Table 3. Interculatc sanduich thichness $d$ for several graphite interculation compounds show ing that the repeat distance $I$, for y lon stuge graphite intercalation compound can be saried over a uide range by proper choice of interculate

\begin{tabular}{|c|c|}
\hline Interculuse & $d$ \\
\hline$L_{1}$ & 3.7110 \\
\hline $\mathrm{Nu}$ & 4.53 \\
\hline $\mathrm{Pr}$ & 4.57 \\
\hline in & 4.57 \\
\hline $\mathrm{Sm}$ & 4.72 \\
\hline Eu & 4.57 \\
\hline$k$ & 5.35 \\
\hline$R h$ & 5.65 \\
\hline ci & 5.94 \\
\hline $\mathrm{Br}$ & 7.14 \\
\hline $\mathrm{HNO}$ & 7.84 \\
\hline $\mathrm{HCIO}$ & 7.44 \\
\hline SO: & 7.96 \\
\hline $\mathrm{Cl}, \mathrm{O}$ & 7.98 \\
\hline Ait. & 8.15 \\
\hline Sht. & 8.46 \\
\hline RuCI. & 9.25 \\
\hline $\mathrm{MnCl}$ : & 9.30 \\
\hline $\mathrm{NiCl}$ & 4.311 \\
\hline Muci. & 9.31 \\
\hline OACl: & 9.35 \\
\hline Fecl. & 4.37 \\
\hline Shci. & 9.42 \\
\hline $\mathrm{CrCl}$ & 4.45 \\
\hline $\mathrm{MnCl}$. & 9.45 \\
\hline CoCl: & 9.50 \\
\hline $\mathrm{FeCl}$ & 9.51 \\
\hline AlCi: & 9.54 \\
\hline $\mathrm{F}: \mathrm{Br}$ & 9.90 \\
\hline FeBi: & 9.95 \\
\hline $\mathrm{KH}_{\mathrm{t}}$ & 10.22 \\
\hline $\mathrm{Re}_{\mathrm{c}}\left(\mathrm{Cl}_{1}\right.$ & 11.78 \\
\hline
\end{tabular}


and at the same potential at which the capacitance measurement had been made, the background current; $I_{b}$, was read off the nanoammeter.

The absolute magnitude of $1_{b}$ increased when the applied potential was stepped in either the cathodic or anodic direction from zero vs the $\mathrm{Ag} / \mathrm{Ag}+$ electrode. The background current became increasingly negative in the cathodic direction and increasingly positive in the anodic direction.

After every experiment, the cell was rinsed several times with distilled HPLC grade acetonitrile and when the cell was dry, it was covered with kimwipes to protect it from dust particles. Then, just prior to an experiment, the cell was again rinsed with distilled HPLC grade acetonitrile but the background current was always present. Other methods of cell cleaning were tried but $i b$ was still present.

The experimental results shown in figure 21 were collected as described earlier, starting at OCP and stepping $50 \mathrm{mv}$ at a time, in this case in the anodic direction. After each potential step an initial quick decay in the current was noticed on the nanoammeter of the potentiostat but then the current was almost steady. It took waiting times of hours for the current to decay significantly. For instance, in one experiment it took one hour for the current to decay from $200 \mathrm{nA}$ to to $10 \mathrm{nA}$. What was causing the background current? 


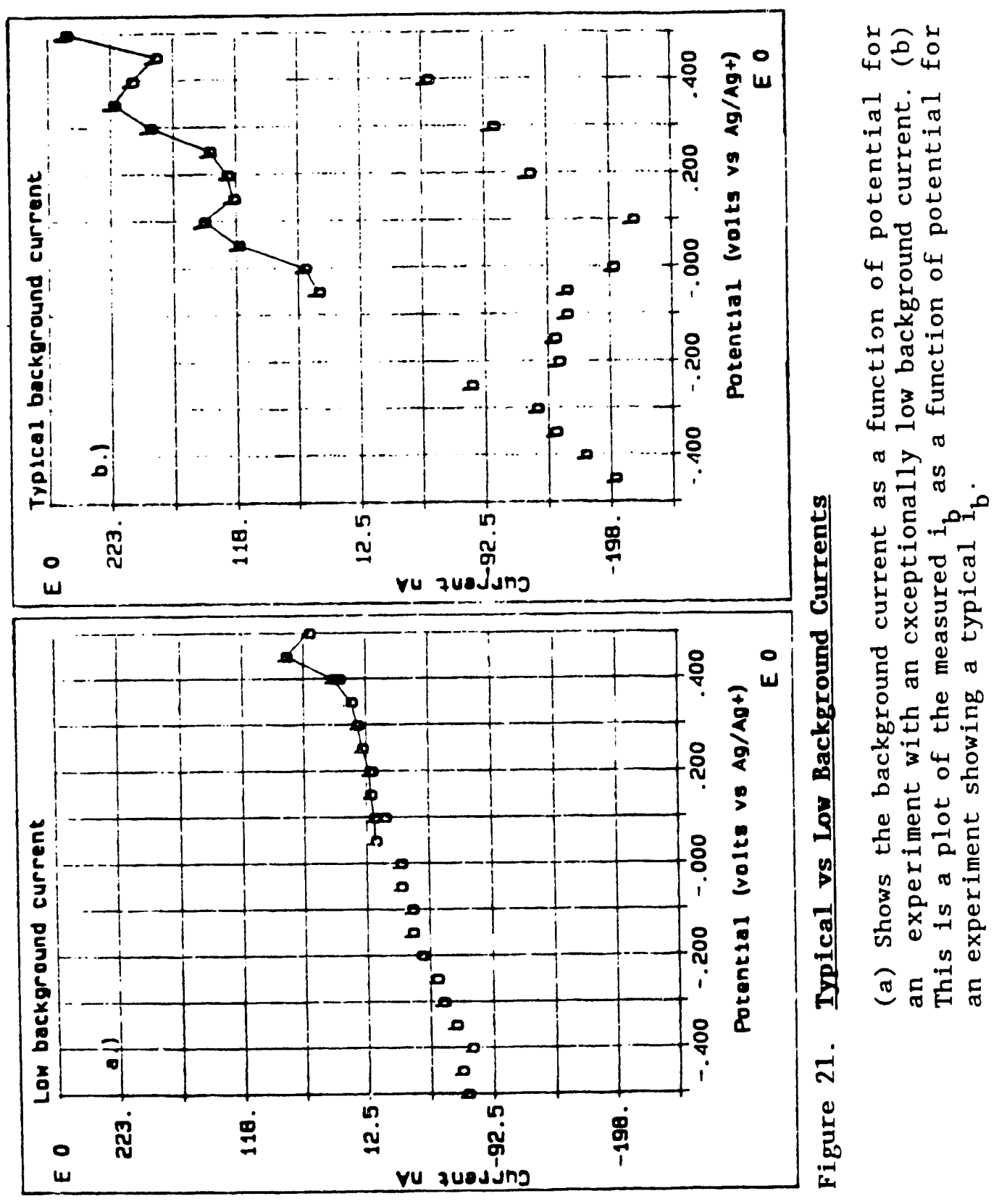


By chance one experiment was conducted, on an electrode with a previous history, which showed an exceptionally low background current, see figure $21 a$. The background current for this electrode, which had received the usual electrode pretreatment procedure, was about one third of that usually seen and didn't show the usual hysteresis between $I_{b}$ for the anodic and the cathodic steps through the same potential range. In figure $21 \mathrm{~b}$ is a similar plot but for an electrode which had a typical background current.

The c/d curves for the exceptionally low background current experiment, at a relatively low background current for this particular experiment: $6 \mathrm{nA}$; and at a relatively high background current for this particular experiment: $80 \mathrm{nA}$, are shown in figure $22 a$ and $b$, respectively. The $c / d$ for another experiment with a typical background current: $145 \mathrm{nA}$, is shown in figure $22 c$. The c/d curve in figure $22 \mathrm{~d}$ has an extremely high corresponding anodic background current. From these $\mathrm{c} / \mathrm{d}$ curves it seems that the curves with the lower background currents have smaller slow components. In spite of the differences, two exponential term fits still yielded the smallest error for all of these $c / d$ curves (as calculated from equation 5 ).

The $c / d$ curve shown in figure $22 d$ is for an electrode pretreated with Vacuum Heat Treatment. This was used to see if VHT would affect the slow process. Surprisingly, electrodes treated in this way showed exceptionally high anodic background 


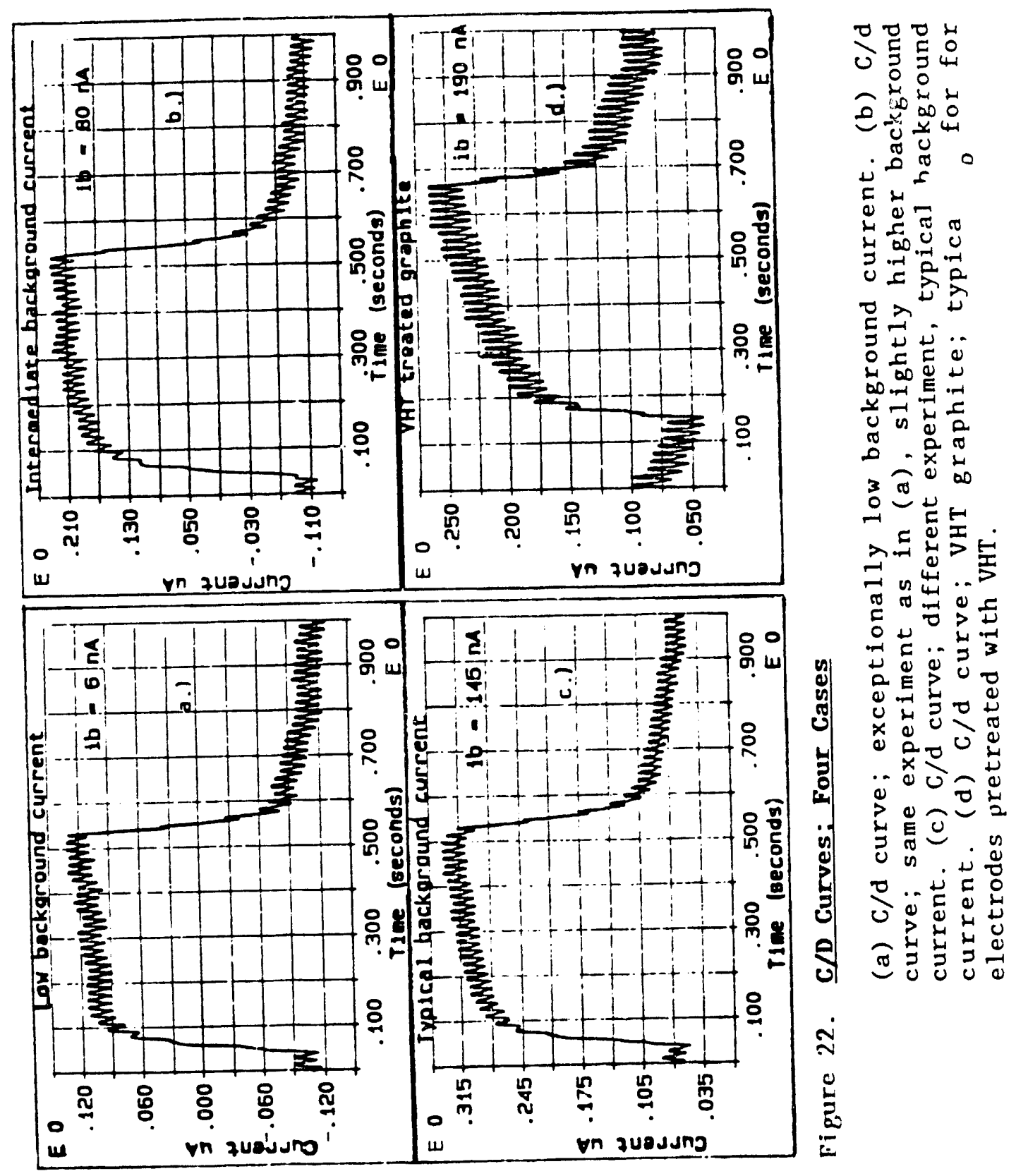


current. In addition to an increase in $I_{b}$ in the anodic region, VHT resulted in an apparent increase in the size and linearity of the slow charging capacitance, this among other effects which will be discussed in further detail later on. In figure 23a, and $23 \mathrm{~b}$ the backgrourd current for the VHT electrode is contrasted with the typical background current for an electrode receiving the usual pretreatment procedures. Since for the VHT treated electrode the $O C P$ was equal to $-0.100 \mathrm{~V}$, the initial step direction was cathodic, (see figure 23b, where the points for the initial cathodic sweep are connected).

Therefore it is still unclear whether or not the background current is a faradaic current but what is clear is that $i_{b}$ contributes to the slow process. Why VHT should enhance the background current and the slow process is unknown, (some possibilities will be discussed later), but the trend of a small slow process with low background current and an increasingly large and steeper slow process with increasing background current is apparent.

To illustrate the relationship between the slow process and the background current more clearly, the results for two electrodes were compared. One of these was that with the exceptionally low $i_{b}$, figure $24 a$ and $b$, and the other was that with a typical $i_{b}$, figure $24 c$ and $d$. The $c / d$ curves were fit as before, using the simplex program. The electrode in 24a and $b$ had 

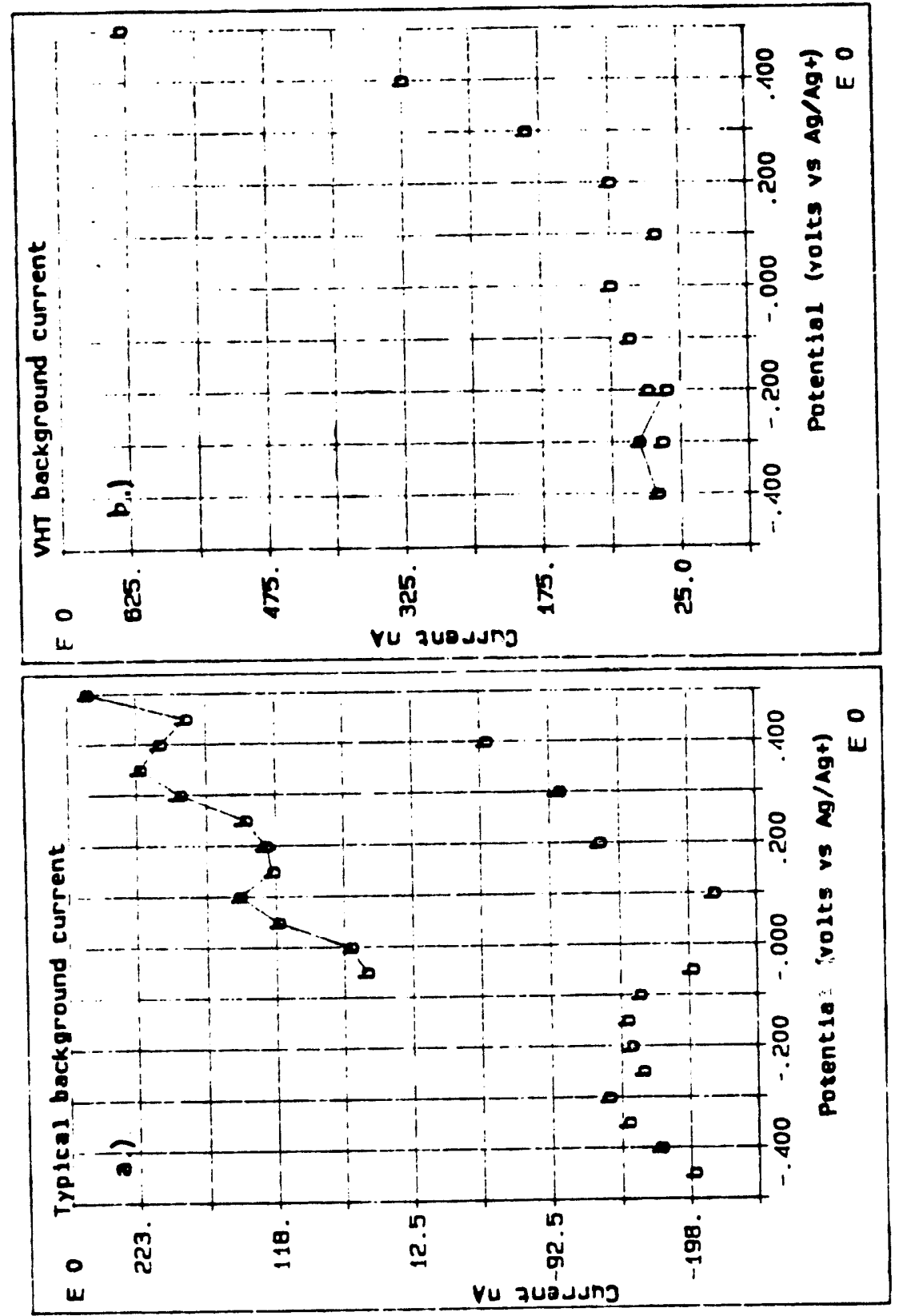

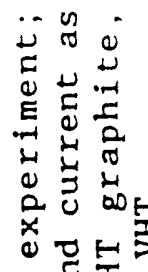

เ

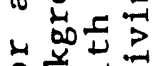

०ै

4 更 30 Ф

出 点

的击

$\circ$ D

造

020

등 훈

एक

대에 $\sum_{4}+0$ ๘ ब 0 め مอ तो 는 选 n 5 응

$3 \rightarrow \frac{1}{4}$ 员 ज $0 \frac{5}{5}$

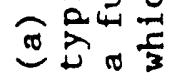




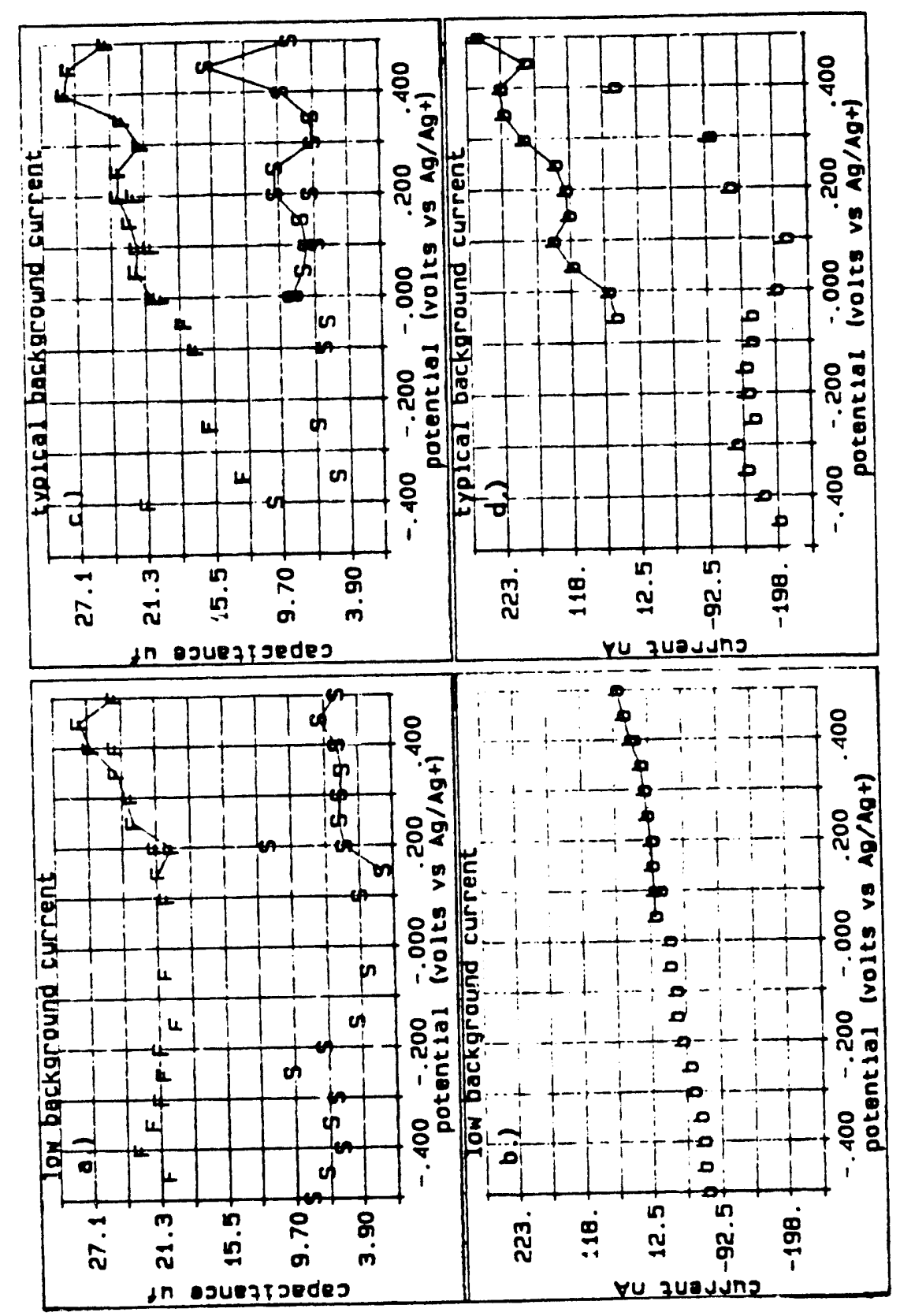

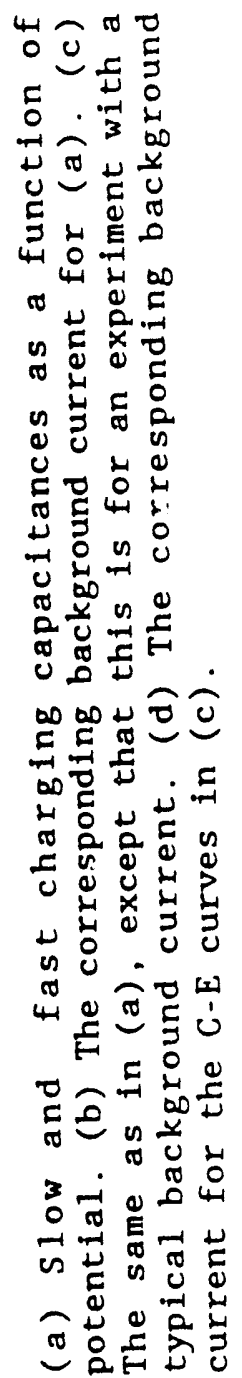

空 
a prior history of having been exposed to potentials outside the safe range and the electrode in $24 \mathrm{c}$ and $d$ was freshly cut. Both electrodes were polished as usual and dried in an oven at $90 \mathrm{C}$. The plots on the bottom half of the figure are of the background current as a function of potential, for the respective electrodes, and those on the top half are plots of $\mathrm{C}_{s}$; (using the symbol $S$ ), and $C_{f}$; (using the symbol $F$ ), as a function of potential.

At each potential a c/d curve is recorded at the most sensitive current range; cr, possible, without causing current overload due to the level of the background current. Sometimes, in order to get a sufficiently large signal for the $c / d$ curve, a waiting time of a few minutes was necessary to allow for $i_{b}$ to decay sufficiently so a more sensitive cr could be used. This was alse important since at more sensitive current ranges the signal to noise ratio became much larger, due to a decrease in the noise level. Waiting for $i_{b}$ to decay was inconsequential with regards to the trends seen in the comparison being made in fic 2 re 24 $\mathrm{b}$ acause the background current was always measured after the $\mathrm{c} / \mathrm{d}$ curve was recorded, and therefore the waiting time shouldn't influence any trend, or lack thereof, seen in this figure.

As before the connected points are the initial anodi': sweep starting at the left most point which was $O C P$, or within $\mathrm{I}^{\mathrm{v}}$ thereof. No dramatic correlation on the order of the nearly 3 
fold increase seen in the background current from one electrode to the other is apparent in the capacitances.

\section{EFFECT OF VHT}

In figure 25, the same comparisons are made between the capacitances of the typical $1_{b}$ data, and VHT data. The same symbol scheme is used here as in figure 24 . It is apparent from this figure that VHT treatment has a dramatic effect on the relative magnitudes of $C_{f}$ and $C_{s}$. $C_{s}$ becomes much larger than typically seen, although the values obtained are not without precedent; see figure $20 \mathrm{~b}$ where values of the slow capacitance of the order of 15 uf were also observed. However, the extremely small values of the fast charging capacitance seen are unique to VHT graphite and the overall capacitance, fast and slow combined, is also smaller than seen previously. This result agrees with that of Fagan, Hu, and Kuwana (10), who also saw a decrease in the electric double layer charging current for glassy carbon electrodes which had received VHT.

VHT is known to decrease the presence of oxygen functionalities on the glassy carbon surface, as has been shown by Fagan, $\mathrm{Hu}$, and Kuwana (10) through X-ray photoelectron spectroscopy. By analogy VHT should decrease the presence of oxygen functionalities on the erige plane of PG electrodes. This could be the 


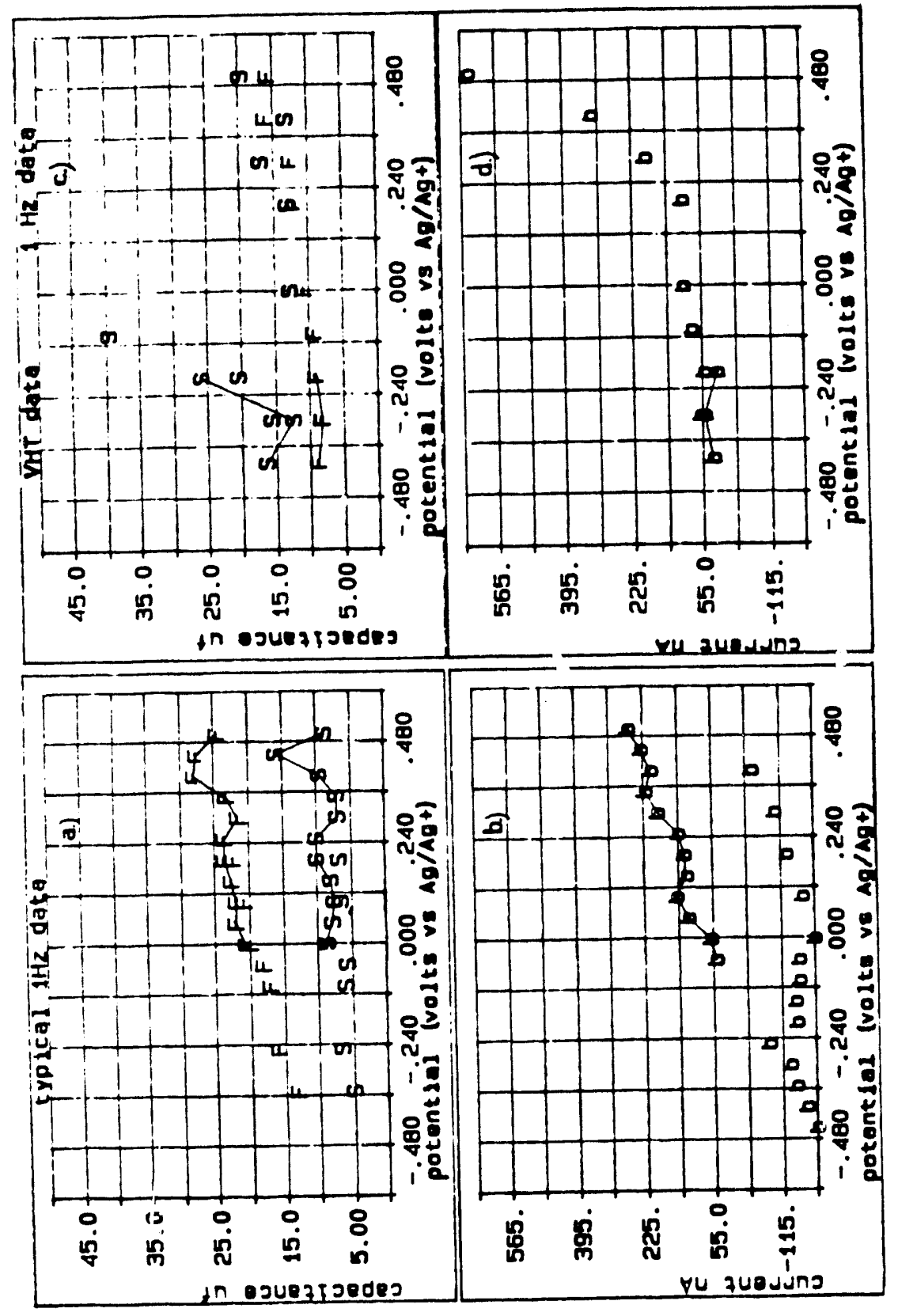

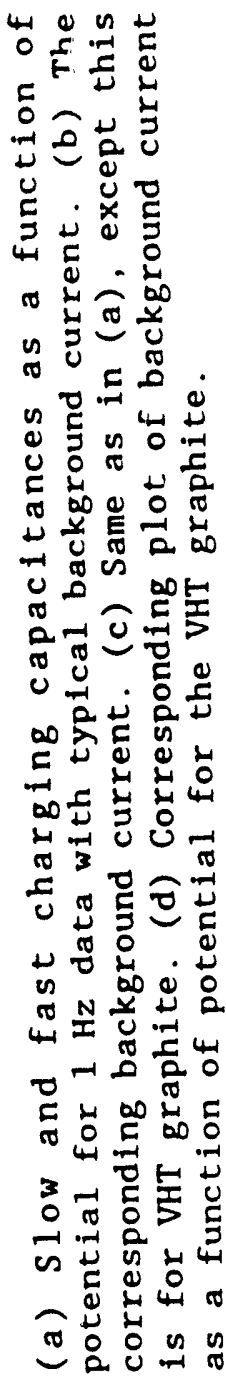

ลัง 
explanation for the overall decreasing capacitance seen. If the Helmholtz-Perrin model is used as an approximate model of the edl (25, p.718), we find that the amount of charge; $q$, stored in the edl is given by:

$$
q-\frac{V \epsilon}{4 \pi d} \quad \text { equation } 6
$$

where $\mathrm{V}$ is the potential difference applied across the electrode, $d$ is the distance between the charge locations in the electrode and in the solution, (each approximating a plate of a parallel plate capacitor), and $\epsilon$ is the permittivity. A decrease in the presence of oxygen on the electrodes surface could result in a decrease in the permittivity and in turn a decrease in the charge the capacitor is able to store, as shown by equation 6 and seen in figure $25 \mathrm{c}$.

\section{EFFECT OF SUPPORTING EIECTROLYTB CONCENIRATION}

To investigate how much of an effect the bulk solution resistance was having on the results, particularly on the slow process, an experiment was run in a $0.4 \mathrm{M}$ TEATFB solution. This solution was four times more concentrated than the usual TEATFB solution. Electrodes received the standard pretreatment; polishing and over. drying. The results for this experiment are 
compared to those of a typical $0.1 \mathrm{M}$ TEATFB experiment in figure $26 ; 26 \mathrm{a}$ for the $0.4 \mathrm{M}$ solution and $26 \mathrm{~b}$ for the $0.1 \mathrm{M}$ solution. In this figure the slow and fast charging capacitances are plotted vs the applied potential. The symbol 4 is used for the $C_{s}$ values and the symbol $F$ for the $C_{f}$ values in 26a; and the symbol 1 is used for the $C_{s}$ values and the symbol $F$ for the $C_{f}$ values in $26 \mathrm{~b}$. These results show an overall increase in the capacitance with an increase in the supporting electrolyte concentration. The slow capacitance increases 2 to 3 fold above the typical values but values of this size have been seen for both VIT graphite and for electrodes with a previous history, see figures $23 \mathrm{c}$ and $20 \mathrm{~b}$, respectively. In contrast, the $C_{f}$ values seen in figure $26 a$ are much larger than those seen for any other experiment. Increasing the supporting electrolyte concentration thus has the effect of increasing the fast capacitance and possibly the slow capacitance. This demonstrates that the rate of charging is influenced by the resistance of the bulk solution.

Is the increase in capacitance seen with increasing supporting electrolyte concentration accompanied by a corresponding increase in the background current? The $C_{f}, C_{s}$, and $i_{b}$ values for the $0.4 \mathrm{M}$ data are plotted as a function of potential in figures $27 \mathrm{c}$ and $d$ and these plots are juxtaposed with similar plots in $27 a$ and b for typical 0.1 M data. Apparently, there is no correlation, the background curient does not show a corresponding increase. 


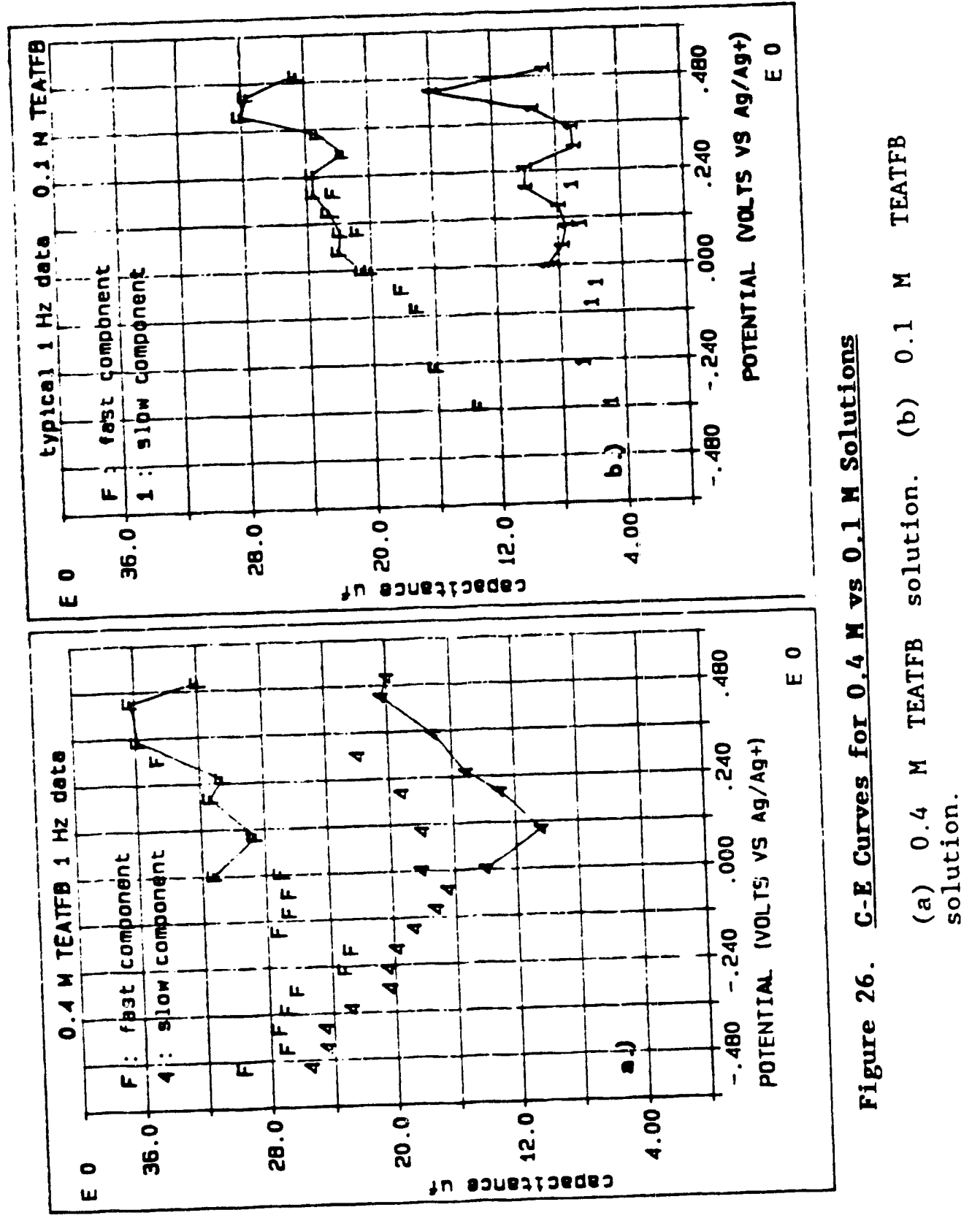




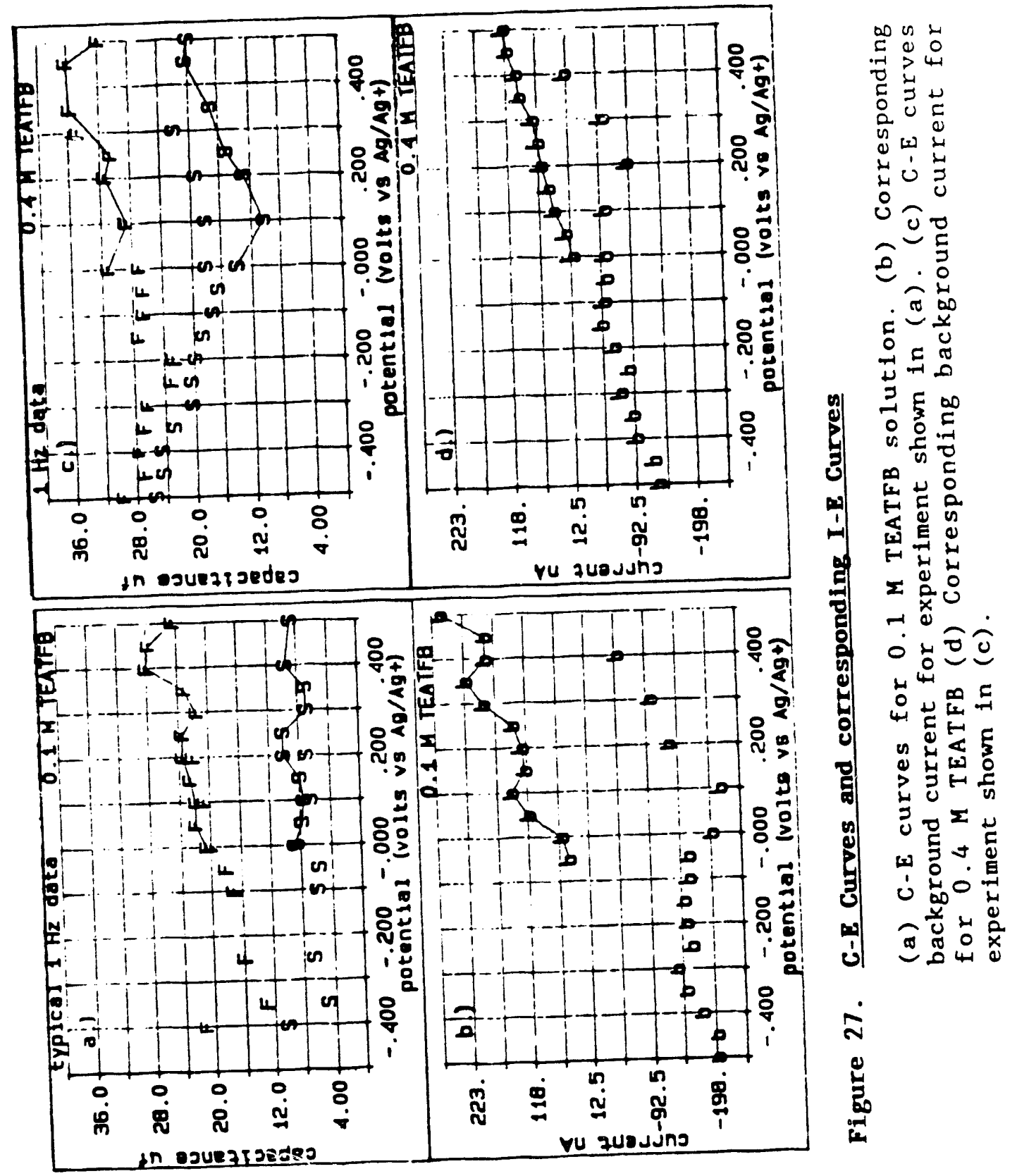


Because TEAt cations are large and their positive charge is centrally located, they are poorly solvated. As a consequence of the lack of a significant solvation sheath they are notorious for undergoing specific adsorption (4). Specific adsorption involves the loss of all solvation by an ion and its coming in direct contact with the electrode's surface, forming the Inner Helmholtz Plane; IHP. The fact that this occurs on carbon electrodes with TEA+ ions, was demonstrated by oren, Tobias, and soffer (14). However, the results shown in figure 27 , to some extent undermine the possibility that the slow capacitance and or the background current could result from the specific adsorption of TEAt cations. If they did, an increase in the background current might be expected with the increases in the supporting electrolyte concentration and the slow and fast processes and this is not seen.

\section{EFFECT ON THE RESISTANCES}

In figure 28 plots illustrating the dependence of the fitted resistances on the applied potential are shown. In $28 a$ and $b$ the plots are for a low background current experiment, slow and fast charging resistances, respectively. In figure $28 \mathrm{c}$ and $\mathrm{d}$, similar plots are made for an experiment with a typical $i_{b}$. For plots of the corresponding background currents see figures $24 \mathrm{~b}$ and $24 \mathrm{~d}$, respectively. The resistances in figure 28 are represented with 


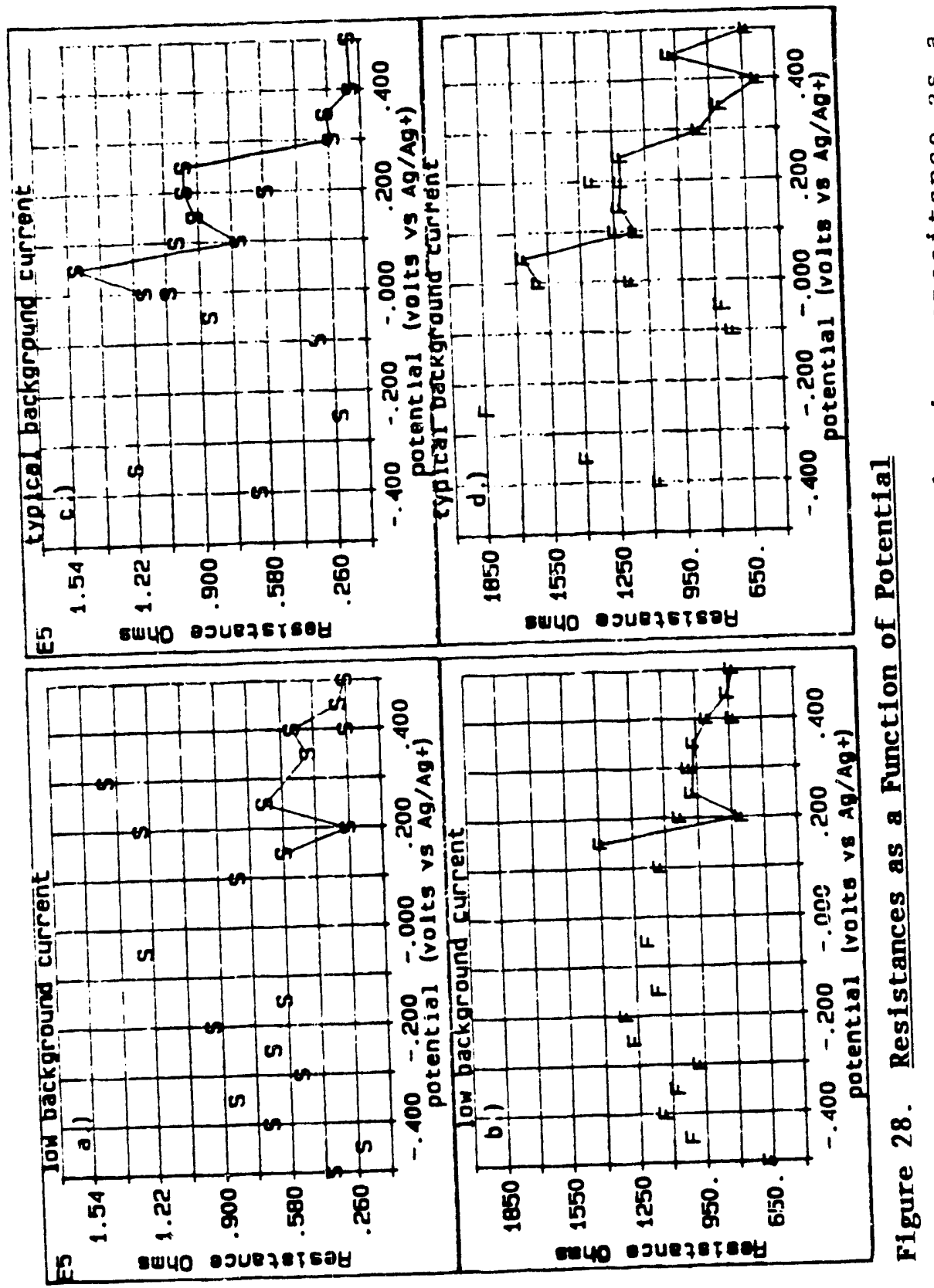

幽导岕

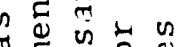

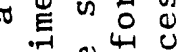

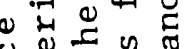

迥行

ก $x_{0}^{x}+\stackrel{0}{0}$

$山 1400$

U $0 \propto ⿻$

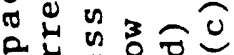

๙

U U 0 . . E

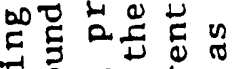
Do 0 ob 4 แ đ

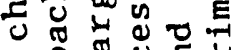
○纪 030 尔 क $\rightarrow$ य हु० \& L 0 。

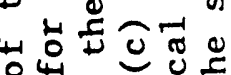
$44_{4} \underbrace{}_{-1}$ 0 บำ क क क

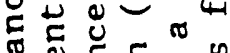

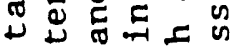

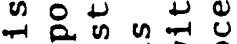

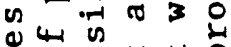
凹ั (1)

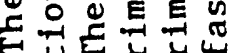
का सा ชิ 
the symbols $F$, for the resistance of the fast process, and the symbol $S$, for the resistance of the slow process. The fast process characteristically has a smaller resistance than the slow process by approximately two orders of magnitude. The resistances show $a$ potential dependence which for the initial anodic sweep has a stroig inverse relationship with the background current. The trend seen in figures $28 a$ and $b$ is less pronounced than that in figures $28 \mathrm{c}$ and $d$; this correlates with the magnitude of the background current, since $i_{b}$ is smaller for the experiment depicted in figures $28 \mathrm{a}$ and $b$ than for that in $28 \mathrm{c}$ and $d$. The reverse sweep just scatters the values, which could have something to do with the hysteresis seen in the background current data.

A similar plot made for the typical $i_{b}, 0.1$ M TEATFB resistance data juxtaposed with the typical $i_{b}, 0.4 \mathrm{M}$ TEATFB data can be found in figure 29. The same trends are seen here but both sets of resistances for the $0.4 \mathrm{M}$ data are much smaller than those for the $0.1 \mathrm{M}$ data. This is in keeping with the idea that the resistance of the bulk solution is playing a role because otherwise a difference between the $0.1 \mathrm{M}$ and the $0.4 \mathrm{M}$ resistances should not be present.

If the background current is a faradaic current it would make sense that the resistances would decrease with increasing background current. This would be the case, since the rate of charging wouldn't be dependent on fons moving through the 


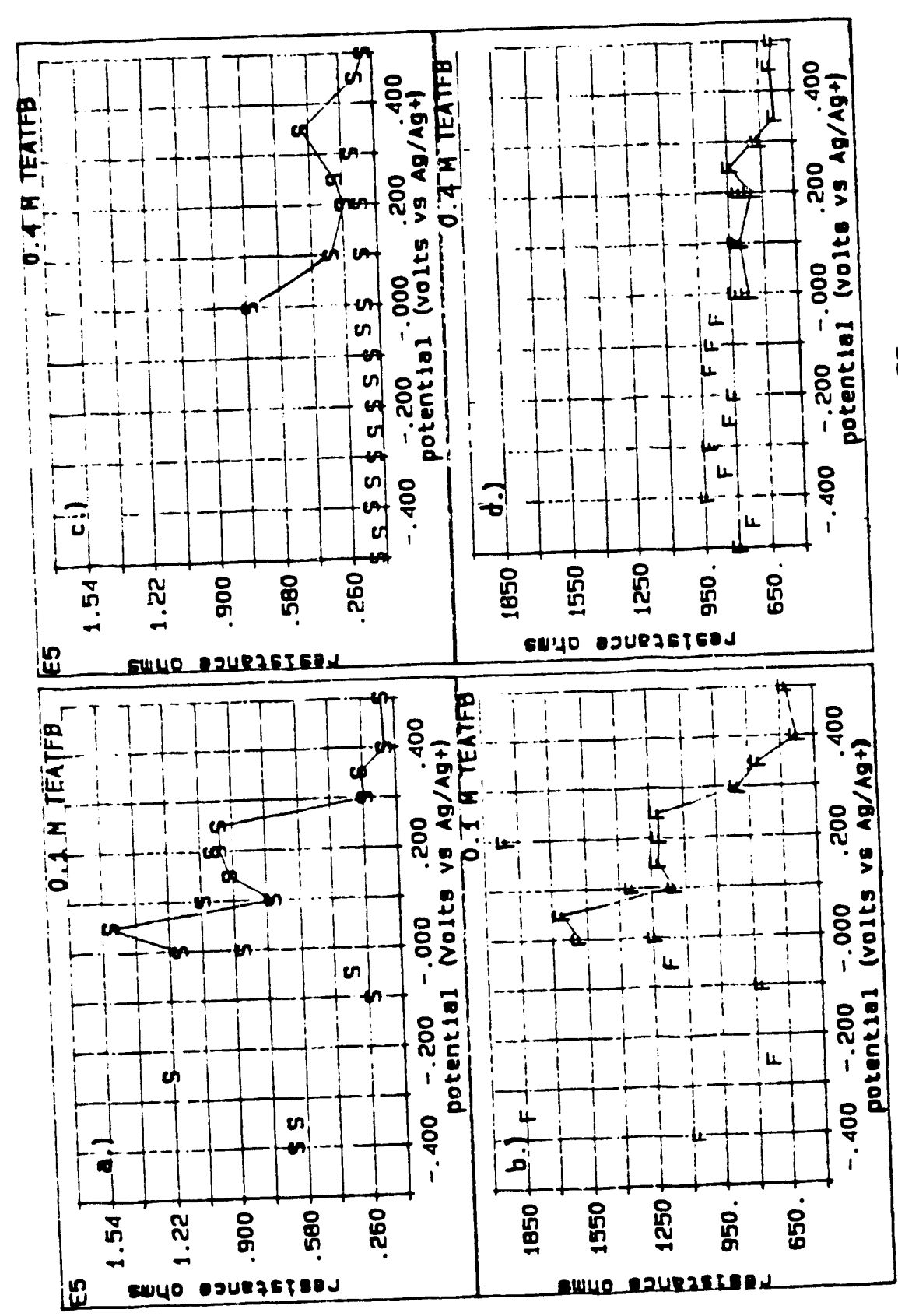

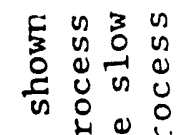

造造

๙

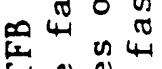

돈 0

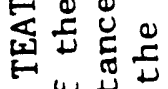

$=40$

20
-10

원

덩 ฮِ

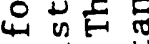

o vo

记

Uू.

造密

3 E

을

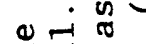

这

山㟔至苩

○崖出

क

ปूة

为出

v 50

苫

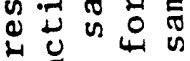

ه

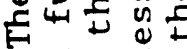

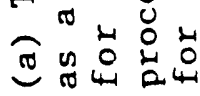


internal structure of the electrode but rather electrons would move easily across the double layer to oxidize or reduce some contaminant. But, being as the resistances were obtained from fitting a funct: on to the data which knew nothing of a faradaic current, how can this trend be explained? There is no obvious answer to this question, however, it may be that the function that would fit a faradaic current has a similar form to the capacitive charging current function used. For instance consider the Erying equation shown below:

$i_{n e t}=n_{S A K}, h\left(C_{0} e^{-\alpha n F\left(E-E^{0^{\prime}}\right) / R T}-C_{R} e^{(1-\alpha) n F\left(E-E^{0^{\prime}}\right) / R T}\right]$

equation 7 (26, p.306).

This equation is for a reversible reaction, where the forward reaction is a reduction and the reverse reaction is an oxidation. The terms are: $n$ is the number of electrons involved in the electron exchange reaction; $F$ is the faraday constant; $A$ is the electrode area; $\mathrm{K}_{\mathrm{s}, \mathrm{h}}$ is what is known as the standard heterogeneous rate constant; $C_{R}$, and $C_{O}$ are the concentrations of the reduced and oxidized states of the electrochemical species, respectively; $\alpha$ is what is known as the transfer coefficient and is $\leq 1.0 ; \mathrm{R}$ is the gas constant; $\mathrm{T}$ is the temperature; $\mathrm{E}^{0^{\circ}}$ is the formal potential of the couple; and $E$ is the applied potential. This equation is not meant for a TVS input but for a 
potentiostated potential; E. However, if one considers the possibility that at a given potential of the TVS, part of the slow process is faradaic and defined by this equation, then how can equation 4 fit the data? For example, in the case of an oxidation, as the applied potential increases in the positive direction the exponent with $C_{R}$ as a coefficient becomes larger and the exponent with $C_{O}$ as a coefficient becomes smaller. The result is a larger negative current. This contradicts the convention used throughout this thesis, where oxidation gives rise to a positive current, but this is just a convention which some electrochemists reverse. Thus, as the applied potential increases the numerator of the exponents in equation 7 increase but there is no way to increase the numerator of the exponential in equation 3 , so, as a consequence to compensate the denominator of the exponent in equation 3 is decreased. More specifically, the resistance is decreased.

Since the level of $i_{b}$ has such a dramatic effect on the shape of the slow process, the slow process must, at least partially, have the same cause as the background current. Whether or not this is a faradaic current still can not be established. On the contrary, the slow charging capacitance shows so little correlation with the background current that it seems likely that it is due to something else as well.

FREQUENCY DEPENDENCE OF THE CAPACITANCES 
If the slow process is due to a slow charging internal structure, it should show a dependence on the frequency of the TVS. The dependence which it should show is, $C_{s}$ should be smaller at higher frequencies. To investigate this, different frequency TVS(s) were used: $1 \mathrm{~Hz}, 5 \mathrm{~Hz}, 10 \mathrm{~Hz}$, and in some cases $100 \mathrm{~Hz}$. The $c / d$ curves were recorded at these frequencies over the range of potentials, the curves were $f i t$, and the corresponding $C_{f}(s)$ and $C_{s}(s)$ were plotted as a function of potential for these frequencies. The results are shown in figures 30 and 31 .

For the $0.1 \mathrm{M}$ solution the fast and slow capacitances for 1 $\mathrm{Hz}, 5 \mathrm{~Hz}$, and $10 \mathrm{~Hz}$ TVS(s) are shown in figure $30 \mathrm{a}$ and $\mathrm{b}$, respectively. As usual, the experiment started at the left most point of the connected points and proceeded as described earlier. No frequency dependence is apparent in plot 30 a but a strong inverse correlation is seen between the frequency and the slow capacitance values in figure $30 \mathrm{~b}$.

The $1 \mathrm{~Hz}$ data and the $10 \mathrm{~Hz}$ data were collected simultaneously, one charging curve was recorded directly after the other at a given potential. Unfortunately, the $5 \mathrm{~Hz}$ data was collected in a different experiment, using a different electrode. In spite of this fact, the trend is believed to be valid. Especially, considering that the $5 \mathrm{~Hz}$ data fits well in between the $1 \mathrm{~Hz}$ and $10 \mathrm{~Hz}$ data.

Figure $30 \mathrm{~b}$ shows a dramatic decrease in the slow charging capacitance with increasing frequency. This result can be 

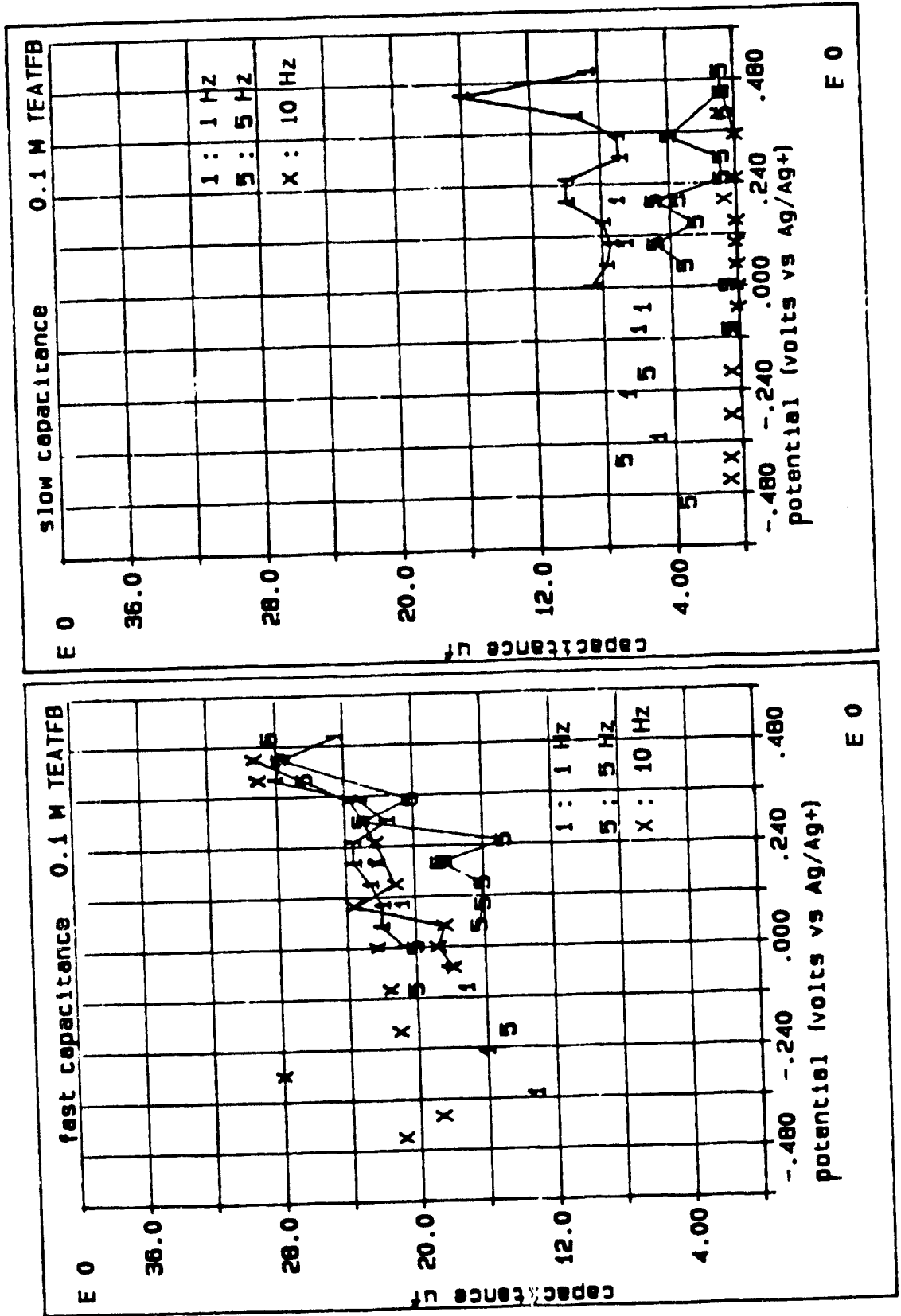

(1) 3 in

$\stackrel{N}{1}$

的-

+已 is

- . .

4 娄

可是

七四

DF

$5 \Sigma 4$

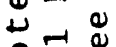

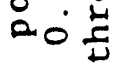

出

4

두

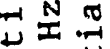

U。

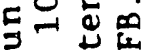

은

क

n...

क ⿻上丨

- I 둥.

un

क

叫

Un-

$\pi$ on

o.

of is o 어

$\rightarrow 0$

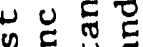

\%

स 3 .

宁

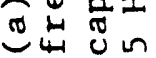

吕 

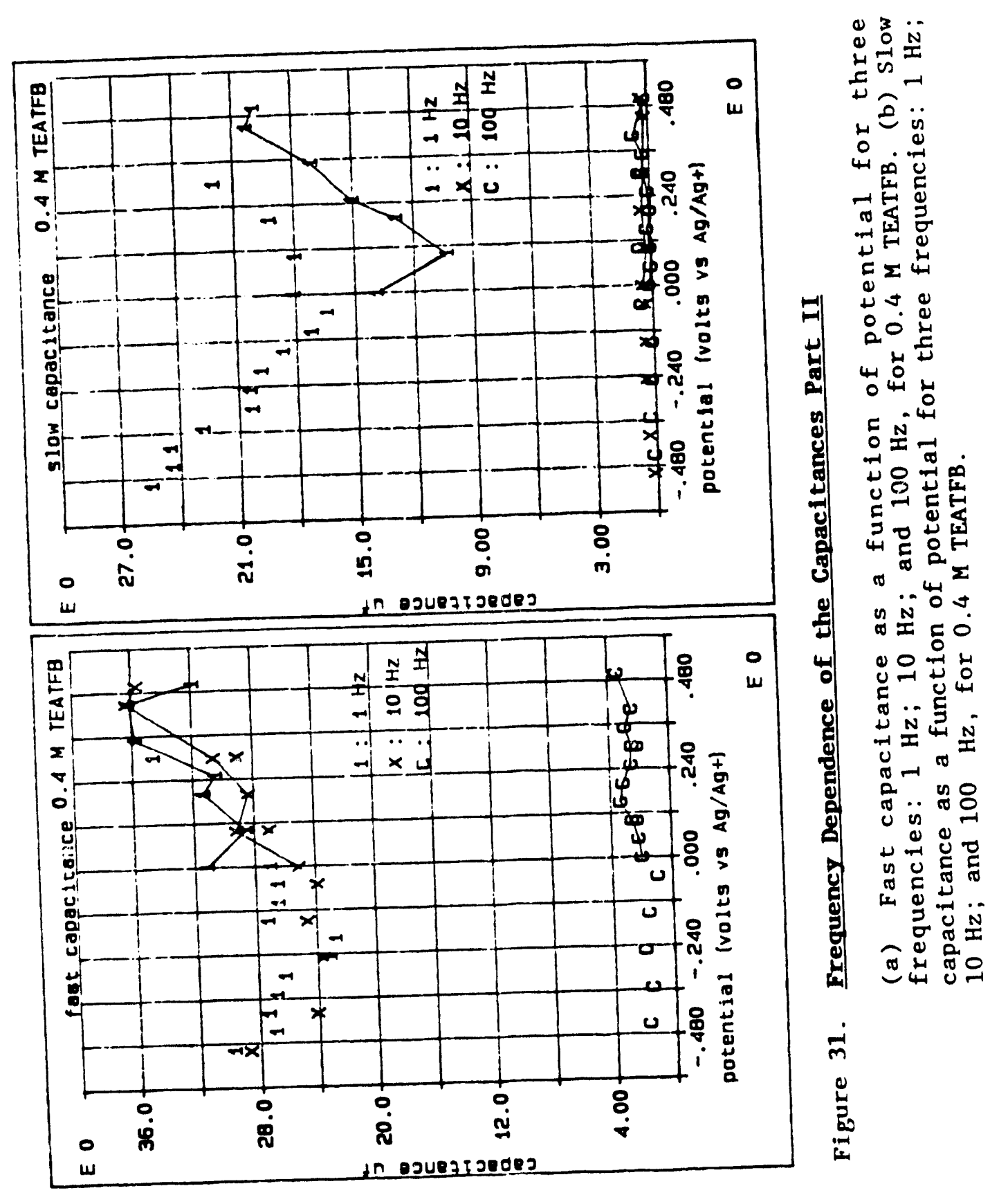
explained by the idea of a distributed capacitance, the higher frequency allowing less time for charging of the less accessible parts of the electrode surface. This affects the slow process more since its RC constant is larger than the period of the input waveform to begin with, and as the frequency of the TVS increases , or in other words as the period of the TVS decreases, this difference becomes greater. Consequently, less of the internal structure is accessible during a scan.

The same trend is seen in figure 31, which is for $0.4 \mathrm{M}$ TEATFB data. The frequencies used in this figure were: $1 \mathrm{~Hz}, 10$ $\mathrm{Hz}$, and $100 \mathrm{~Hz}$. The most important fact revealed in $31 \mathrm{a}$ is that even the fast capacitance shows a frequency dependence at high enough frequencies. This supports the idea that the slow process is a capacitive process because both the slow process and the fast process show a similar frequency dependence. In order to fit the $100 \mathrm{~Hz}$ data only half of the charging or discharging curve could be used. In other words the curve that was fit had to start from an uncharged state and not from a charged state equal and opposite to the charging caused by the immediate half of the TVS. If the whole charging or discharging curve were used unreasonable values for the capacitance were obtained.

\section{CONCLUSIONS:}


The capacitance of the edl of a semiconductor is often modeled as three capacitances in series (4). These capacitances are those of the space charge in the semiconductor; $C_{s c}$, that of the diffuse double layer; $C_{\text {diff }}$, and that of the Helmholtz plane; $C_{H}$, and they combine to yield the total capacitance: $C_{\text {tot }}$ according to the follow: $g$ equation:

$$
c_{\text {tot }}=\frac{1}{c_{s c}}+\frac{1}{c_{\text {diff }}}+\frac{1}{c_{H}} \quad \text { equation } 8
$$

However, if the two capacitances seen in this work, distinguished as a slow and a fast capacitance, were separate capacitances of equation 8 , one would expect them to charge at the same rate as one another. Capacitances in series can't charge at independent rates. Therefore, a conclusion which can be reached by the fact that the sum of exponential terms, equation 4, fits the data is that, if both charging processes are capacitive, the capacitances which are measured and distinguished as a fast and a slow capacitance are in parallel. Randin and Yeager (11) model the capacitance of the edge plane of pyrolytic graphite and the capacitance involved with charging in the microfissures as a sum of many series resistance, and capacitance circuits in parallel. This seems to be the behavior which the fit of the data implies, which suggests that the microfissures might be invclved. If the distributive capacitance 
which is measured here is due to the microfissures, this may be the first time it has been measured.

Further conclusions which can be reached $f: 0 m$ the fit obtained are: that the fit using equation 4 appears to be unique, except for the values of $a_{1}$, and $a_{2}$, the sum of which is unique. And that the same set of parameters can be used to fit the charging and the discharging sections of the curve, implying that the charging process is reversible.

Evidence seen through experiments conducted thus far supports the argument that the background current is a faradaic current and that it can be enhanced through VHT. Specifically, the evidence is: that the background current shows no direct correlation with the $c_{s} 0: C_{f}$ values; that when $i_{b}$ is large the c/d curve looks like case d of figure 10; the inverse relation between $i_{b}$ and the resistances of the initial sweep (see figures 28 and 29), the possibility of explaining this with the Erying equation; and the fact that VHT enhances $i_{b}$.

$I_{b}$ may be caused by a faradaic current from water cortamination. Water could be introduced into the system through the acetonitrile, which may not be adequately purified. VHT could enhance $i_{b}$ and the slow process, by increasing the activity of the graphite electrode, which it is known to do (10). This would cause more water $t r$ be oxidized and consequently, the increase in the anodic background current and in the size and linearity of the slow process. 
The effect of VHT on $C_{f}$ can be explained as described earlier, using the Helmholtz-Perrin model. VHT through its reduction of the presence of oxygen functionalities, could cause a decrase of the permittivity; $\epsilon$, resulting in a decrease in the charge stored in the edl capacitance.

Finally, the behavior seen with $0.4 \mathrm{M}$ TEATFB solution implies that the $0.1 \mathrm{M}$ solution is not concentrated enough. Otherwise, the four fold increase in concentration shouldn't have decreased the fitted resistances so drastically. Other resistances than the resistance of the buik solution, for instance the resistance involved with ions entering the internal structure, ideally should be the limiting resistances. 


\section{APPERDIX 1:}

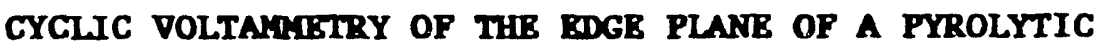 GRAPHITE ELECTRODE IN ACETONITRILE.}

Cyclic voltammetry is an experimental electrochemical technique in which a TVS, usually on the order of a few hundred mvs to a couple of volts in amplitude, is imposed on a working electrode. This is accomplished through the use of a three electiode cell and a potentiostat. The three electrodes serve the following purposes. The working electrode is where the chemistry of interest is occurring and therefore the potential across its interface is the potential which is controlled. This potential is controlled by placing a reference electrode as close as possible to it and measuring the potential difference between these two electrodes. If the potential difference is not the desired potential difference, the potentiostat changes the applied potential via the auxiliary electrode until the measured potential difference is that desired. The current flowing through the working electrode is measured and this is then plotted as a function of potential. This plot is known as a cyclic voltammogram; cv. 
Before any other progress using the edge plane, PG electrode could be made in this study, a clean edge plane, PG, cv had to be obtained in the electrolyte solution of interest. The solvent of interest is acetonitrile, since it can be used over a wide potential range, both anodic and cathodic limits are reported to be due to the discharge of the supporting electrolyte in all cases. To start with, the supporting electrolyte was tetraethylammonium tetrafluoroborate; TEATFB. Not only did a clean $\mathrm{cv}$ have to be obtained but its features, for this solvent, supporting electrolyte zystem, had to be determined from experiments and incomplete information in the literature.

Despite what seemed to be every possible precaution to maintain a contaminant free environment, at the outset a peak was observed. This peak usually appeared as a single spike but sometimes the corresponding reduction half was detectable (see figure 32). The position of the spike fluctuated between approximately $-0.200 \rightarrow 0.100 \mathrm{~V}$. This peak appeared in the return sweep of a $c v$ which started at $E_{i}=+0.125 \mathrm{~V}$ and scanned to a switching potential $E_{f^{-}}-0.700 \mathrm{~V}$, (see figure 32). Several authors, doing work with aqueous electrochemistry and carbon or graphite electrodes, have explained peaks which they have seen near or in this region by attributing them to the presence of oxygen functional groups on the edge plane (11, 26-29). 


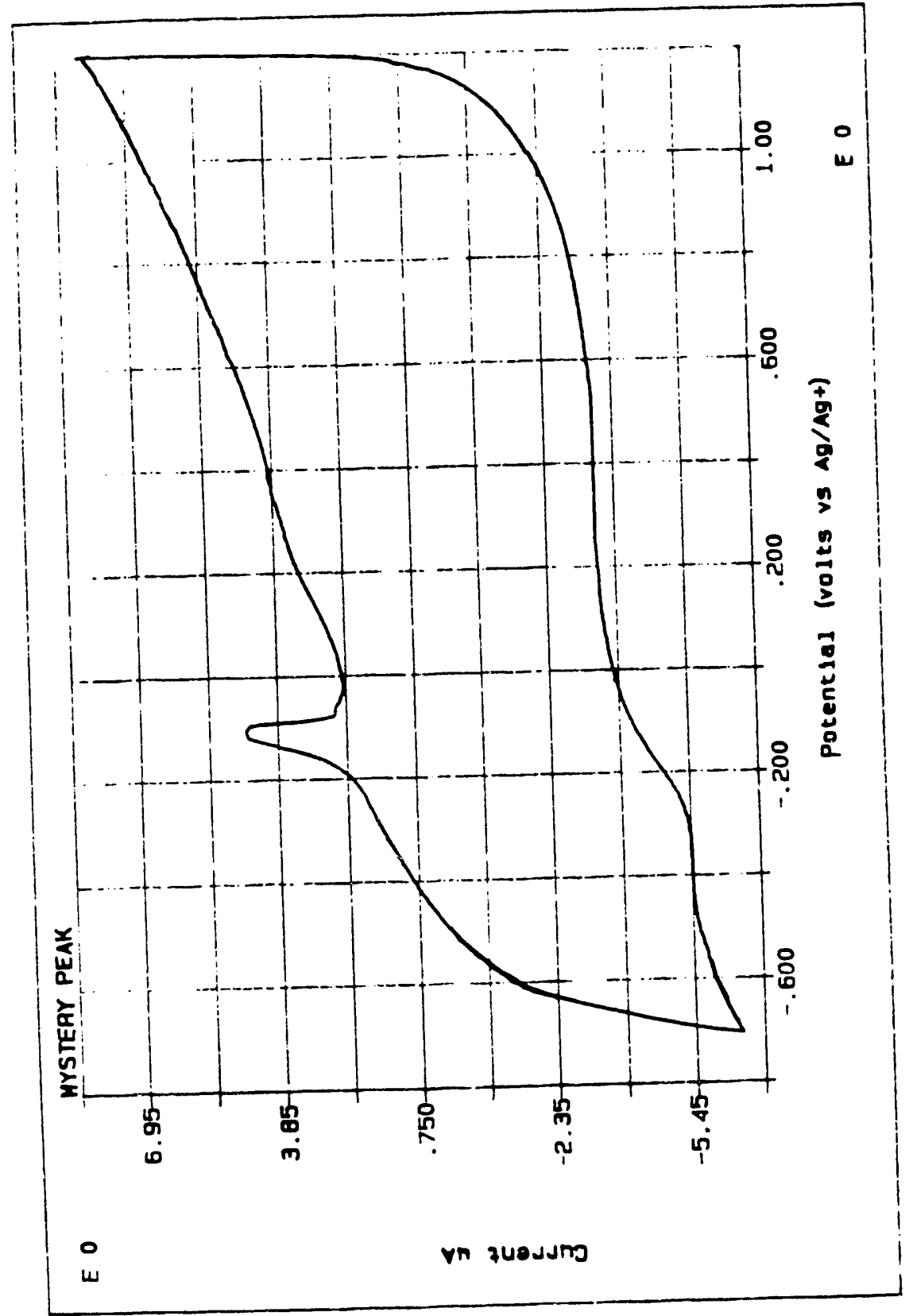

(

4

잉

3.

in

เป

3 in

过

$\stackrel{0}{\perp}>$

은

$\pm \stackrel{2}{-1}$

$>$

응

‥

$0 \pm$

1 盛

战

范

के

노요 은

ํํㅇ $\stackrel{0}{0}$

2.

S.

늠웅

岗 
A considerable amount of work has been done on identifying and characterizing the oxygen functional groups on carbon and graphite materials (30-33). Carboxyls, phenols, carbonyls; figure 33a, quinones; figure 33b, hydroquinones, and ether structures have all been postulated. Of these, the quinone/hydroquinone couple is the most frequently used to explain peaks in what should otherwise be clean cvs (11, 26-29). Phenol and carboxyl groups are not offered as possible redox sites on the electrode surface because the redox reactions of molecules with corresponding functional groups are usually followed by irreversible chemical reactions in aqueous solutions and this doesn't seem to be the behavior seen (33).

The quinone group is believed to undergo the following reaction:

$$
2 \mathrm{H}^{+}+2 \mathrm{e}^{-}+\text {quinone } \rightarrow \text { hydroquinone }(11,26-29,34)
$$

Randin and Yeager (11) report the position of what they believe to be a peak due to this couple at approximately $0.6 \mathrm{~V}$ vs RHE, which is close to $0.0 \mathrm{~V}$ vs the reference electrode used for this work. They also report that this peak shifts $60 \mathrm{mv} / \mathrm{pH}$ unit, which implies that these groups may be in different chemical environments, depending on what other surface oxides, etc, they are 

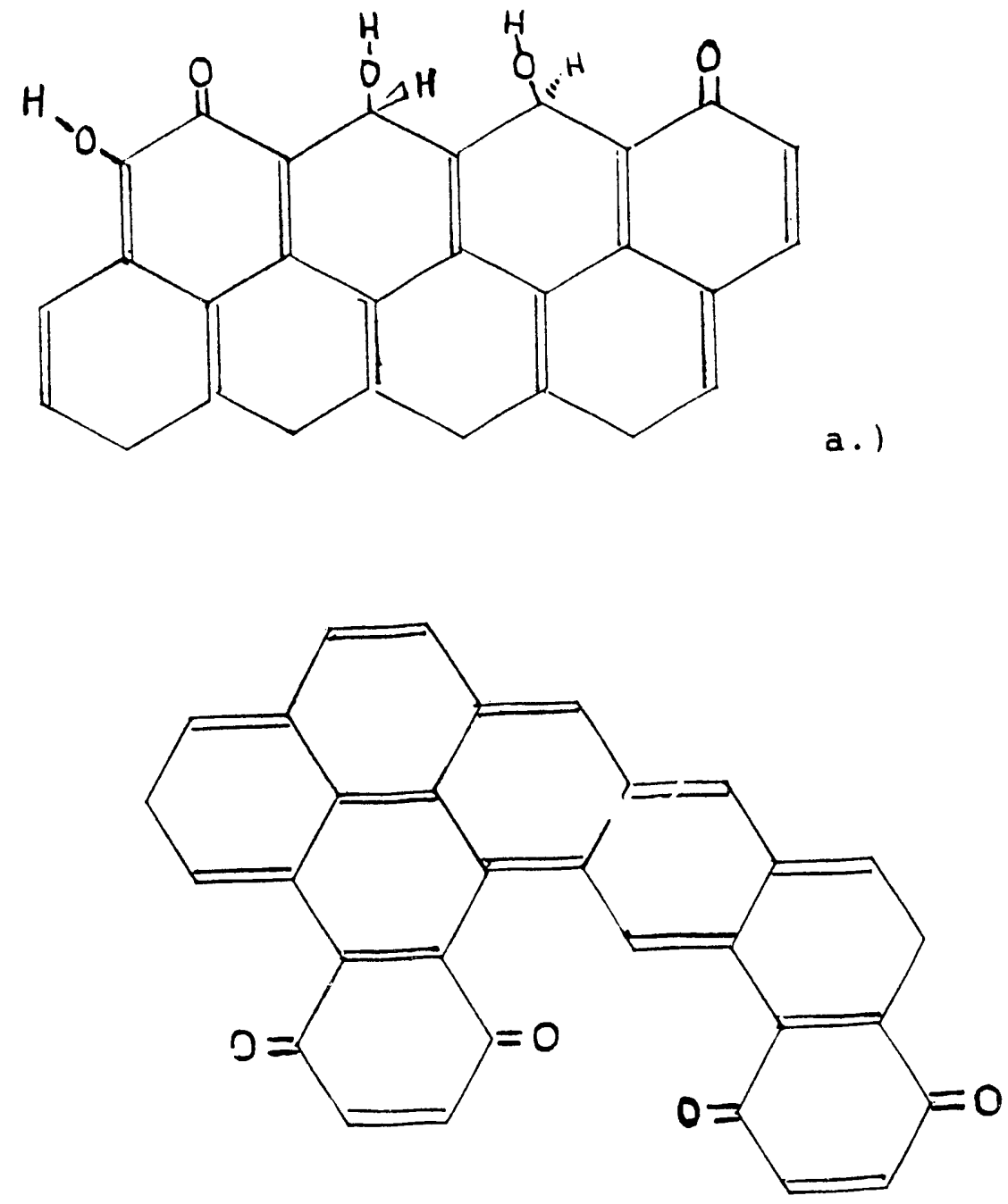

b.)

Figure 33. Oxygen Functionalities

(a) Carboxyl, Phenol, Carbonyl groups. (b) Quinone group. 
surrounded by. Different environments leading to different peak potential positions, depending on the $\mathrm{pK}_{\mathrm{a}}$ of the group.

As pointed out and investigated by Nagaoka and co-workers $(35,36)$, the reduction of quinone to hydroquinone shouldn't occur in aprotic solvents such as acetonitrile. In spite of this, Nagaoka and co-workers, working in AN with supporting electrolytes of cations: $\mathrm{Na}^{+}, \mathrm{Li}^{+}, \mathrm{K}^{+}$, etc, recorded cvs similar to those seen in aqueous solutions, in that a small reversible couple appeared at: -0.320 to $-0.230 \mathrm{~V}$ vs $\left(\mathrm{Ag} / 10 \mathrm{mM} \mathrm{AgNO}_{3} ;(\mathrm{AN})\right.$ $0.1 \mathrm{M}$ TEAP), on a glassy carbon; GC, working electrode and a carbon fiber working electrode. They believed "that the interaction between the carbon surface and the metal ions occurred because of the uptake of the ions into the micropores" of the carbon electrode (36). Nag,aoka and co-workers verified this through the use of flow through electrolysis, which involved monitoring the change in the cation concentration of the solution leaving cile cell with a dropping mercury electrode stationed just outside of the cell. The concentration changes they measured were greater than those which would correspond to monolayer coverage on the external surface of the electrode and they offered this as evidence that the micropore structure was involved.

Aware of these results but wanting to first check for other possibilities for the source of the peak, the possibility of contamination was considered first. Since, the electrodes are polished in a $\mathrm{dH}_{2} \mathrm{O} / \mathrm{d} . \mathrm{p}$. slurry (see the experimental section), 
maybe $\mathrm{dH}_{2} \mathrm{O}$ had been adsorbed by the electrode. To test for this, electrodes received the usual pretreatment procedure, except at the end they were stored under a vacuum of $\leq 10$ utorr for 3 hrs. After letting the cell sit OCP for approximately an hour, the peak appeared as before.

The fact that letting the cell sit OCP for an hour seemed to produce the peak, suggested two possibilities, that the working electrode compartment maybe leaking and this peak was due to something on the stainless steel;s.s., insert, or that something was being adsorbed from the solution onto the graphite electrode. The immediately obvious candidates for an adsorbed chemical species were: water from the solvent, (despite the purification steps taken, see experimental section); water from the supporting electrolyte (known to be hygroscopic), the water could provide the proton necessary for the reduction of quinone to hydroquinone; an organic impurity from the graphite electrode (organic, since any inorganic impurity should have been removed during the extraction in the $\mathrm{MeOH}$ soxhlet, see experimental section).

To check the first possibility a cy was run of s.s. in the working solution from $-0.240 \mathrm{~V}$ to $+1.25 \mathrm{~V}$, at a scan rate of 50 $\mathrm{mv} / \mathrm{sec}$ (see figure 34). No sign of the peak was seen and a characteristic cv different from that which had been seen with the graphite was seen. This is good, since, if these cvs were in- 

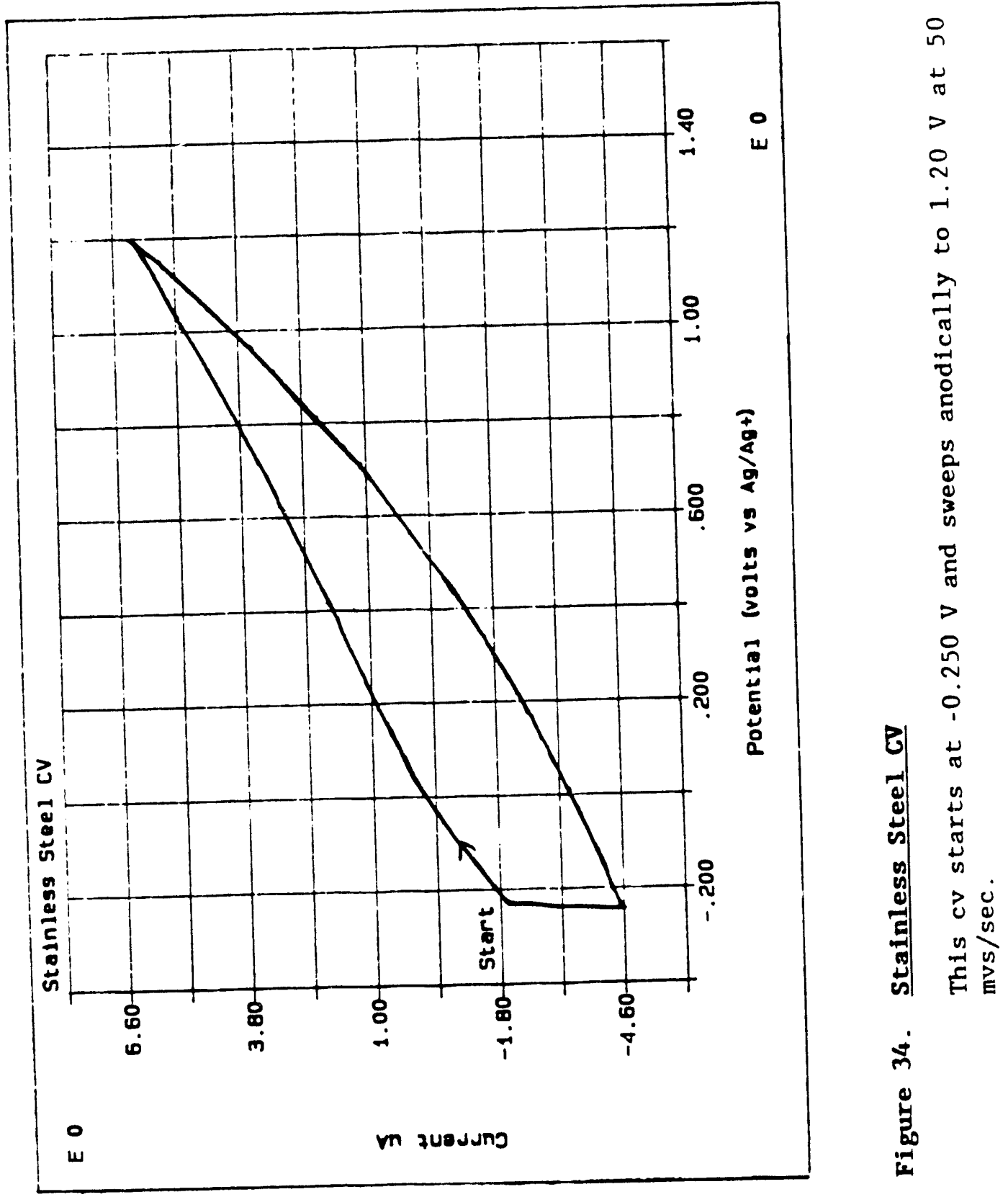
distinguishable and electrochemistry was actually being done on s.s. it would be disastrous.

To check for the possibility of adsorption of an organic impurity, an additional step was added to the pretreatment proceduri just prior to vacuum drying. In this step, the electrodes were extracted in a hexane soxhlet for $10 \mathrm{hrs}$. The peak behaved as before.

Treating carbon electrodes with a radio frequency plasma of $\mathrm{O}_{2}$ is known to enhance the surface population of oxygen containing functionalities and is believed to enhance the surface population of : phenol, carboxyl, and quinone groups especially (34). Since we had a radio frequency plasma generator in our lab (see experimental section for details), all that was required before it could be used was that it was set up with a vacuum line and a tank of UHP grade, $\mathrm{O}_{2}(\mathrm{~g})$.

PG electrodes were pretreated as before except for the last step prior to insertion in the electrochemical cell; during which they were treated with an $\mathrm{O}_{2}$ radio frequency plasma. Disappointingly, no change in the $c v$ was apparent with regard to this peak. One difference which was seen was in the Open Circuit Potentials of electrodes treated in this way (see appendix 4).

The peak had still not been identified but a few things had become apparent through the investigation which had been conducted so far. From the shape of the cv, it appeared that the reduction peak was diffusion dependent, whereas the oxidation 
peak was due to something on the surface of the electrode. This is evident by the broad appearance of the reduction peak and the spike like appearance of the oxidation (see figure 32). Secondly, no peaks are seen at all until a potential is reached sufficient to reduce the species. This can be seen in the series of cvs shown in figures $35 a, b, c, d$, and $e$, where in $35 a$ the initial potential, or starting potential; $E_{1}, 1 s-0.500 \mathrm{~V}$ and the potential is scanned at $100 \mathrm{mv} / \mathrm{sec}$ to a switching potential of $0.000 \mathrm{~V}$. In this sweep the oxidation peak shows up at approximately $-0.200 \mathrm{~V}$ and after reaching $0.00 \mathrm{~V}$ the potential is swept back in the opposite direction. In scan $35 \mathrm{~b}$ the electrode has been at a potential sufficiently negative for reduction for longer: during a large portion of the return sweep from the previous scan and for the beginning of the present scan. Consequently, the oxidation peak is larger because thore is a greater concentration of the reduced species at the surface of the electrode. If $E_{1}$ is then increased by $+200 \mathrm{mv}$; figure $35 \mathrm{c}$, the peak no longer grows since less time is spent at a potential sufficiently negative for reduction and therefore less time is available to build up a concentration of the reduced species on the surface of the electrode. Finally, if $E_{i}$ is stepped up an additional $+100 \mathrm{mv}$ the peak grows smaller and on the second scan through this potential range, the peak disappears, scans $35 \mathrm{~d}$ and 35 , respectively. 


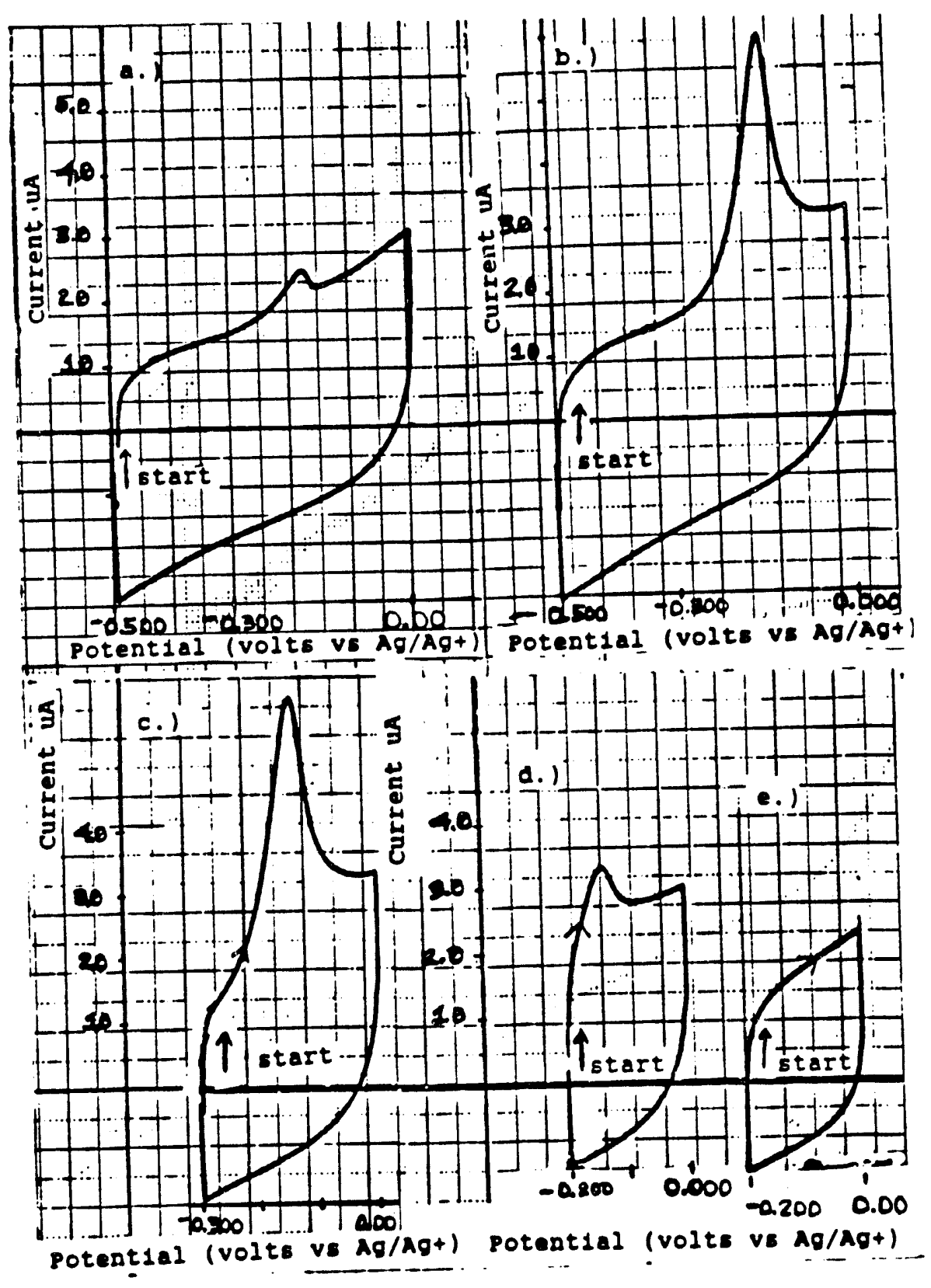

Figure 35. What is Apparent: Series of CV(s)

(a) Scan at $100 \mathrm{mv} / \mathrm{sec}$, from $-0.500 \mathrm{~V}$ to $0.00 \mathrm{~V}$. (b)

Repeat of scan (a). (c) Scan at $100 \mathrm{mvs} / \mathrm{sec}$, from

$-0.300 \mathrm{~V}$ to $0.00 \mathrm{~V}$. (d) Scan at $100 \mathrm{mvs} / \mathrm{sec}$, from

- $0.200 \mathrm{~V}$ to $0.00 \mathrm{~V}$. (e) Repeat of scan in (d). 
The third thing which had become apparent was that the only way to consistently produce this peak was to leave the cell assembled and at OCP for a few hours. These three things, in concert with the facts that the peak was sometimes near and sometimes at identically $0.0 \mathrm{~V}$, and that the electrode surface often appeared a light shade of gray after an experiment, suggested that maybe the reference electrode was leaking and that $\mathrm{Ag}$ metal was plating on the graphite.

To check this, the cell was allowed to sit assembled for 2 hrs at OCP and then a $c v$ was run to see where the peak fell see figure 36a. Only a very small peak is visible just negative of zero. Next, the solution was spiked with approximately $1 \mathrm{ml}$ of $0.1 \mathrm{M} \mathrm{AgNO}_{3}$; (AN) solution, the peak was enhanced and showed the same characteristic spike for the oxidation that had been seen previously, see figure $36 \mathrm{~b}$. This wasn't conclusive evidence that it was due to silver ion but the fact that the peak wasn't seen again after the junction of the reference electrode was changed is more conclusive.

At this time, the junction of the reference electrode had been a Vycor tip supposedly sealed to a Pyrex tube via shrinkable Teflon tubing. Obviously, the leak rate from this junction was too high, either from around the tubing due to a poor seal, or through the Vycor itself. Experiments conducted to measure the flow rate through Vycor were troublesome but seemed to suggest that the leak wust be from around the tubing. Repeated attempts 

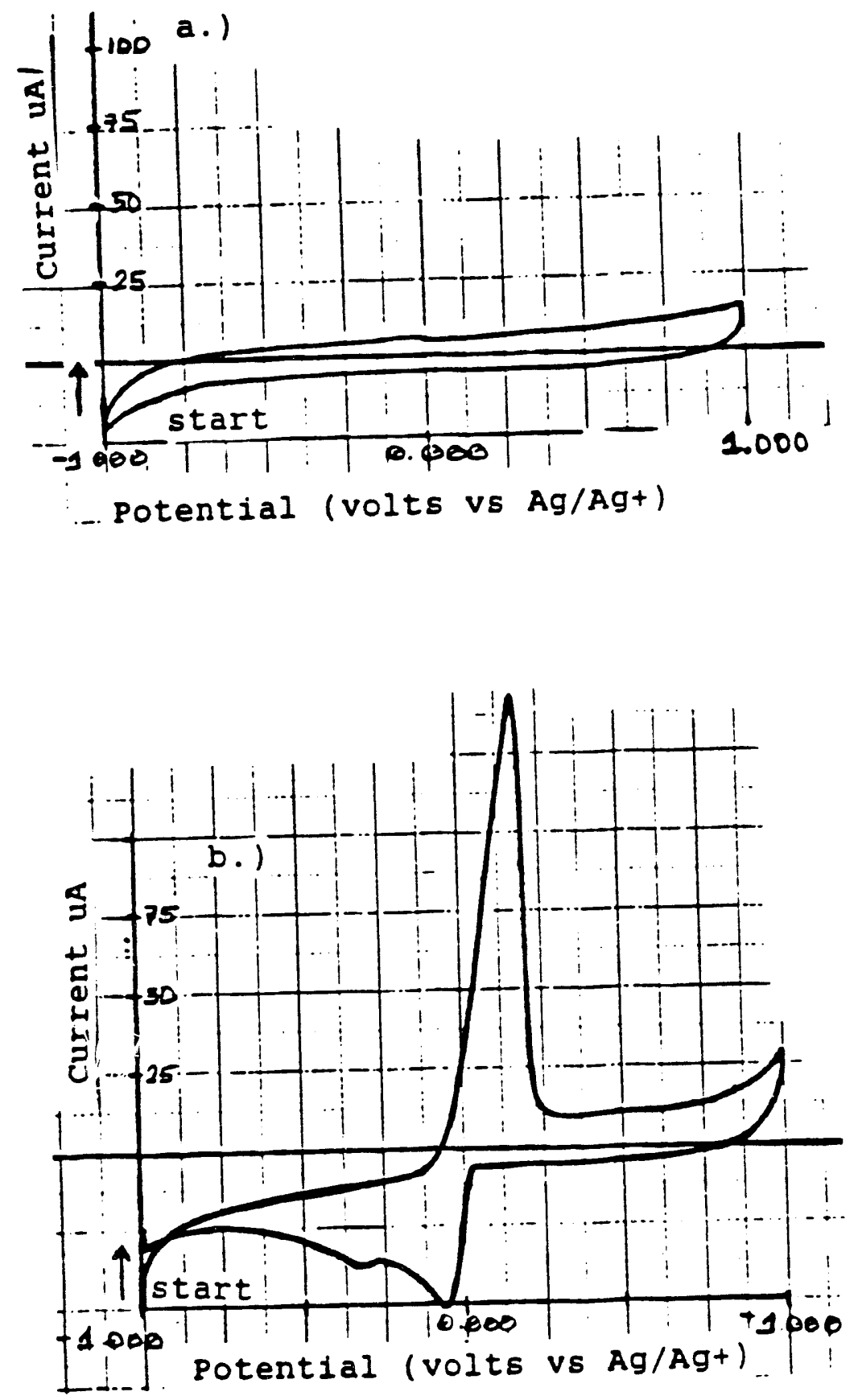

Figure 36. Spike with AgNO Solution

(a) Cyclic Voltammogram before spike, showing small peak around zero volts (scan rate $100 \mathrm{mv} / \mathrm{sec}$ ). (b) Cyclic Voltamogram after spiking with a few mls of $0.1 \mathrm{M} \mathrm{AgNO}_{3}$ solution. 
were made to improve the seal; double layers of tubing, matching the diameters of the pyrex with that of the vycor more closely, but no success was met. As a result the junction was changed.

According to Sawyer $(7, p .28)$ the commonly used junctions with the slowest leak rates are soft glass in pyrex, quartz in pyrex, or a platinum wire "sealed" in pyrex, (the quotation marks here are because if this electrode is properly made a space should exist between the wire and the pyrex glass large enough so that the electrode has a leak rate of approximately $3-30 \mathrm{ul} / \mathrm{hr}$ and therefore it isn't actually sealed). One possible problem with these junictions is that "too low a flow rate may lead to erratic junction potentials or a tendency of the junction to clog if the sample contains colloidal materials." ( 7, p.26). "For long term experiments where interference is most likely", (interference from contamination), "an intermediate solution compartment can be employed." (37, p.340), see figure 37 ( 7, p.55). This may be used in the future, with possible small changes to the reference electrode and its compartment's design, and of course the same cell and working electrode designs shown in the experimental section. 


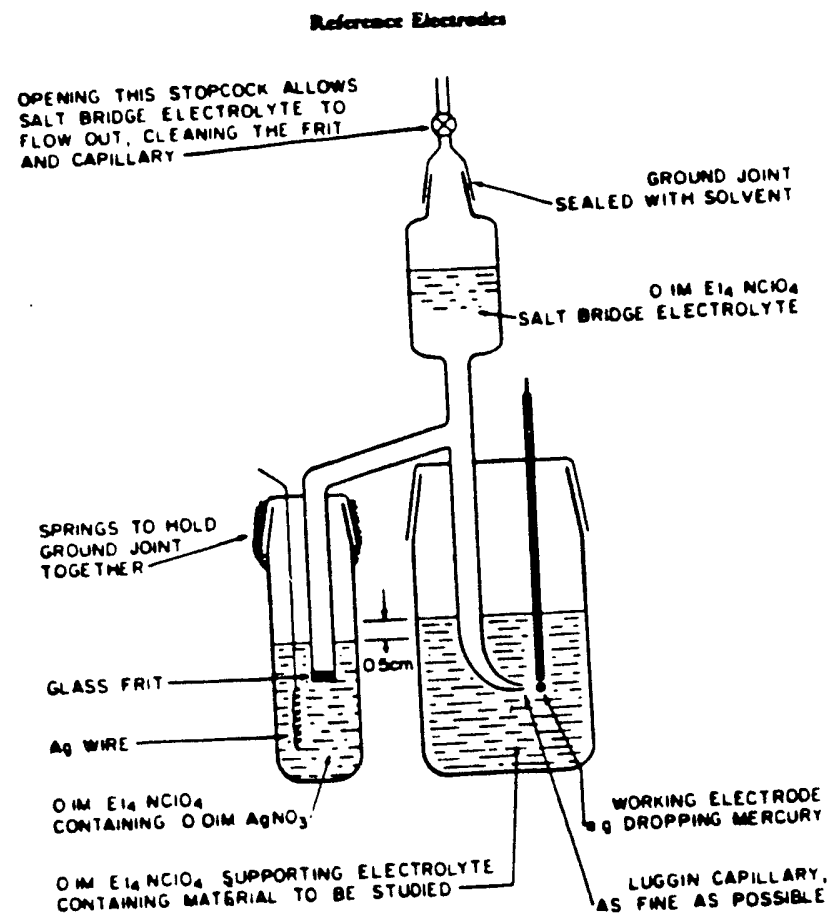

\section{Figure 37. New Reference Electrode Junction}

This figure illustrates a means of preventing $\mathrm{Ag}+$ from leaking into the solution without causing a problem because of a clogged or excessively slow rate of transfer across the junction. Reprinted with permission from Sawyer, D.T.; Roberts, J.L. Experimental Electrochemistry For Chemists; Wiley: N.Y. N.Y., 1974; p 55. Copyright (1974) John Wiley and Sons. 


\section{APPENDIX 2:}

\section{WORRING RANGES: 1imits to the sizes of accoptable applied potentials}

Thus, a Pt wire "sealed" in pyrex was used as the junction for the reference electrode and the peak disappeared. The cv now appeared free of any significant current flow between +1.0 and $1.0 \mathrm{~V}$ (see figure 38); above $+1.0 \mathrm{~V}$, something is oxidized and below $-1.0 \mathrm{~V}$ something is reduced. This is peculiar in light of the decomposition potentials for similar solvent and supporting electrolyte systems reported in the literature.

On a DME, in an AN solution, Kolthoff and Coetzee (38) report a working range of -2.8 to $-0.5 \mathrm{~V}$ VS SCE for tetrabutylammonium iodide; (TBAI), and they report a working range of -2.8 to $-0.5 \mathrm{~V}$ vs SCE for tetraethylammonium bromide. The anodic limit is because of the mercury. It isn't specified what determines these ranges but the tetraalkylammonium ion is the most likely compound to be reduced since it is positively charged and the solvent is slightly basic. Both of these potentials for the two different tetraalkylammonium cations are $-2.8 \mathrm{~V}$ vs $S C E$, or approximately $-3.4 \mathrm{~V}$ vs the reference electrode used here. 


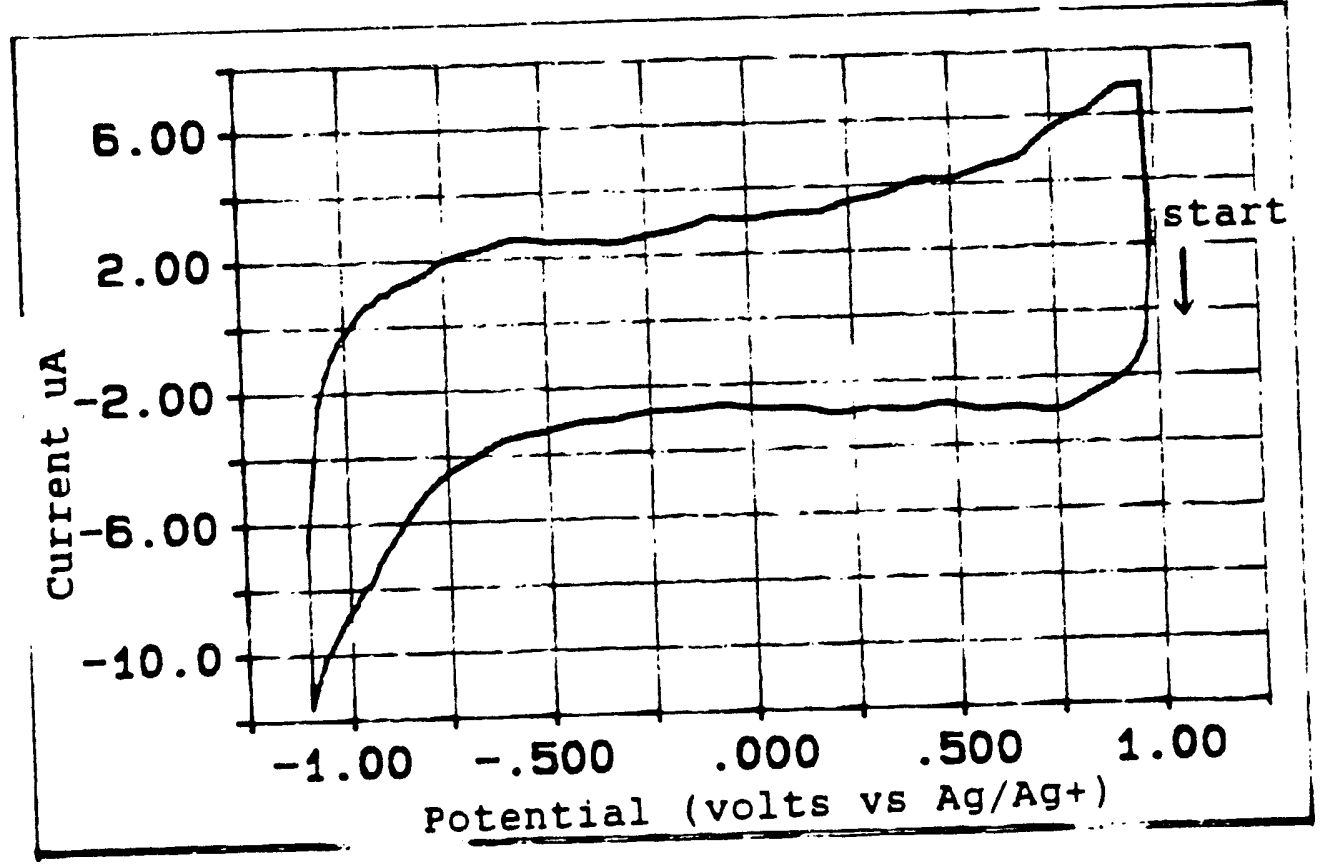

Figure 38 . Clean C.V.

The potential range from $1.00 \mathrm{~V}$ to $-1.10 \mathrm{~V}$ is scanned at $100 \mathrm{mv} / \mathrm{sec}$, with only very small current flow, which appeared at the switching potentials. 
According to Weisslerger (37) an acetonitrile solution with a TBAP electrolyte has a working range of -2.3 to $2.1 \mathrm{~V}$ vs SCE which converts to -2.637 to $1.763 \mathrm{vs} \mathrm{Ag} / \mathrm{Ag}^{+}$. He reports that $\mathrm{TBA}^{+}$ is reduced at a platinum electrode at $-2.30 \mathrm{~V}$ vs SCE and that CLO - Is oxidized at $2.1 \mathrm{~V}$ vs SCE (which determines the upper limit for the range of the AN solution which he reports). This differs by $-0.5 \mathrm{~V}$ from the reduction potential reported by Koltoff and Coetzee for tetrabutylammonium ions. What exactly the perchlorate ion is oxidized to isn't well understood but the following is hypothesized in Weissberger's reference 16 .

$$
\begin{aligned}
& \mathrm{ClO}_{4}^{-} \rightarrow \mathrm{e}^{-}+\mathrm{ClO}_{4} \cdot \\
& \mathrm{ClO}_{4}^{\cdot}+\mathrm{CH}_{3} \mathrm{CN} \rightarrow \mathrm{HCLO}_{4}+\mathrm{CH}_{2} \mathrm{CN} \\
& 2 \cdot \mathrm{CH}_{2} \mathrm{CN} \rightarrow \mathrm{NCCH}_{2} \mathrm{CH}_{2} \mathrm{CN}
\end{aligned}
$$

More relevant, Weissberger reports an oxidation potential for $\mathrm{BF}_{4}^{-}$of $2.91 \mathrm{~V}$ vs $\mathrm{Ag} / \mathrm{Ag}^{+}$. In contradiction to this, Bard (8, p.65) reports that, at a Pt electrode, an $A N$; NaBF, solution has a decomposition potential of $+4.0 \mathrm{~V}$ vs SCE. 
If the most conservative estimate possible is made from this contradictory data, it would seem that the system studied here should be stable from -2.637 to $2.91 \mathrm{vs} \mathrm{Ag} / \mathrm{Ag}+$, with the anodic limit probably being higher. At the edge plane, PG electrode this doesn't appear to be the case.

In the cvs of the edge plane of PG recorded in this work, if the potential excursion was taken beyond approximately $-1.15 \mathrm{~V}$ in the cathodic direction, see figure $39 \mathrm{a}$, or beyond $1.4 \mathrm{~V}$ in the anodic direction, see figure $39 \mathrm{~b}$ the start of large peaks can be seen with currents on the order of $100 \mathrm{uA} / .08 \mathrm{~cm}^{2}$, (the area is the geometric area estimated entirely from the surface dimensions of the electrode). Because of the inconsistencies in the literature and the results seen in this work on graphite, the same system was investigated using a Pt electrode.

The electrode used was a $0.5 \mathrm{~cm}$ length of $0.5 \mathrm{~mm}$ diameter 99.99858 pure Pt wire, (from AESAR/Johnson Matthey inc), sealed in uranium glass with a tungsten lead. This electrode also, like the graphite electrode, had an area of $0.08 \mathrm{~cm}^{2}$ (as calculated from its surface dimensions). Scans were run over different ranges in a $0.1 \mathrm{M}$ TEATFB solution starting with, +0.250 to -0.250 and then: +0.500 to $-0.500 ;+1.00$ to $-1.00 ;+1.00$ to $-1.50 ;+1.25$ to $-1.75 ;+2.0$ to $-2.0 ;-1.5$ to $+2.0 ;$ and 0.00 to 3.00 (see figures $40 a-h$, respectively). In these scans a redox couple was seen in the cathodic region, at an $E_{0}$ value of approximately $1.25 \mathrm{~V}$. The position of this peak is very similar to that seen on 
Figure 39. Applied Potential Linits on Graphite Electrodes

Each figure is a new experiment, fresh, unused
solution and freshly prepared electrodes. (a)
Cathodic limit; scan rate: $100 \mathrm{mv} / \mathrm{sec}$ (b) Anodic
limit; scan rate: $100 \mathrm{mv} / \mathrm{sec}$. (c) Reversibility of the
redox couple at the cathodic limit; scan rate:
$100 \mathrm{mv} / \mathrm{sec}$.



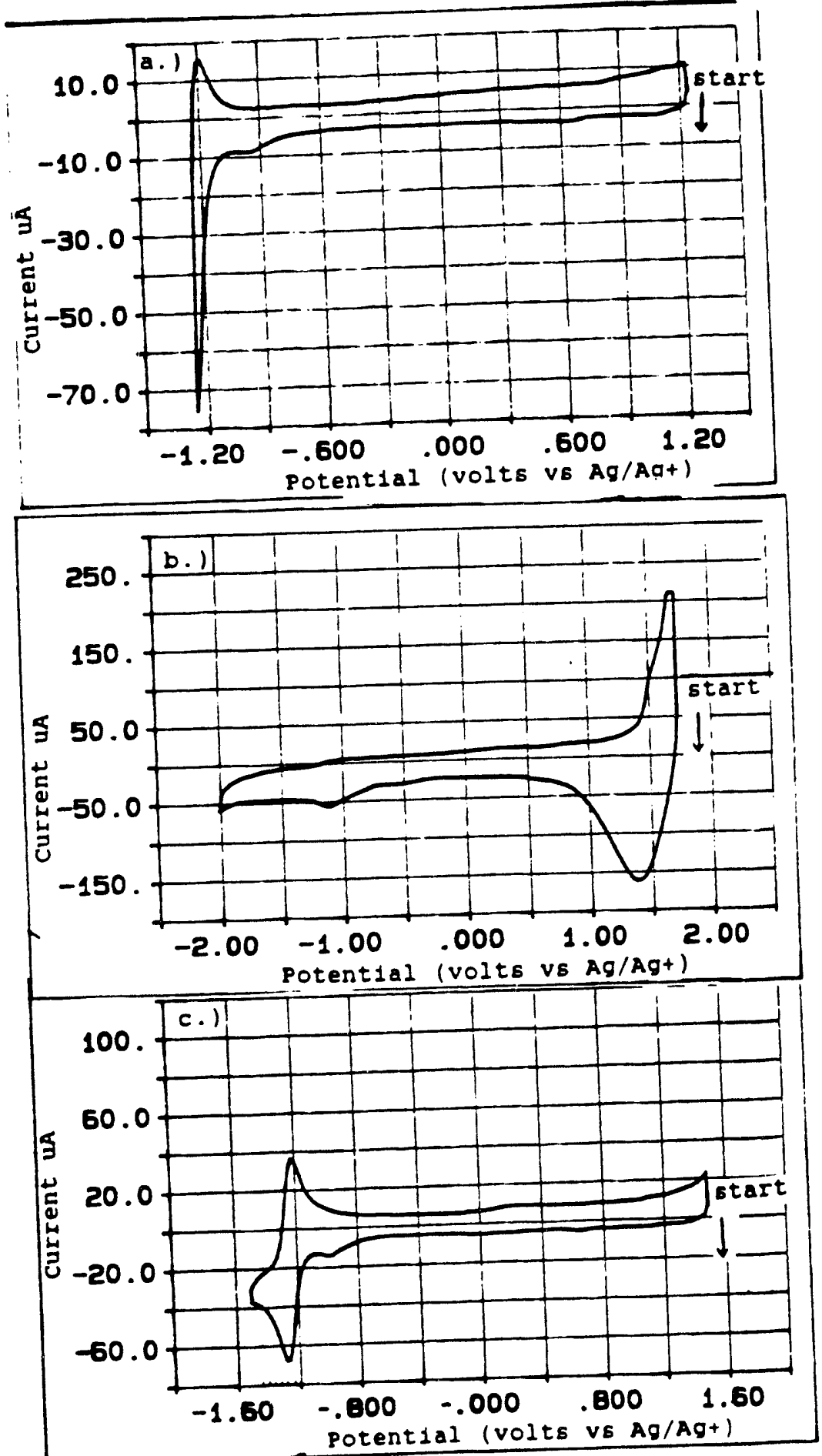
Figure 40. Applied Potential Linits on Pt Electrode

Each figure is a new experiment, freshly prepared solution and freshly prepared electrodes. All scans are at $100 \mathrm{mv} / \mathrm{sec}$. (a) $0.250 \mathrm{v}$ to $-0.250 \mathrm{~V}$ (b) 0.500 $\mathrm{v}$ to $-0.500 \mathrm{~V}$. (c) $1.00 \mathrm{~V}$ to $-1.00 \mathrm{~V}$ (d) $1.00 \mathrm{~V}$ to

$-1.50 \mathrm{~V}$. (e) $1.25 \mathrm{~V}$ to $-1.75 \mathrm{~V}$. (f) $2.00 \mathrm{~V}$ to -2.00 $\mathrm{V}(\mathrm{g})-1.50 \mathrm{~V}$ to $2.00 \mathrm{~V}(\mathrm{~h}) 0.00 \mathrm{~V}$ to $3.50 \mathrm{~V}$ (i) $1.20 \mathrm{~V}$ to $-1.75 \mathrm{~V}$. (j) $1.75 \mathrm{~V}$ to $-1.75 \mathrm{~V}$. 

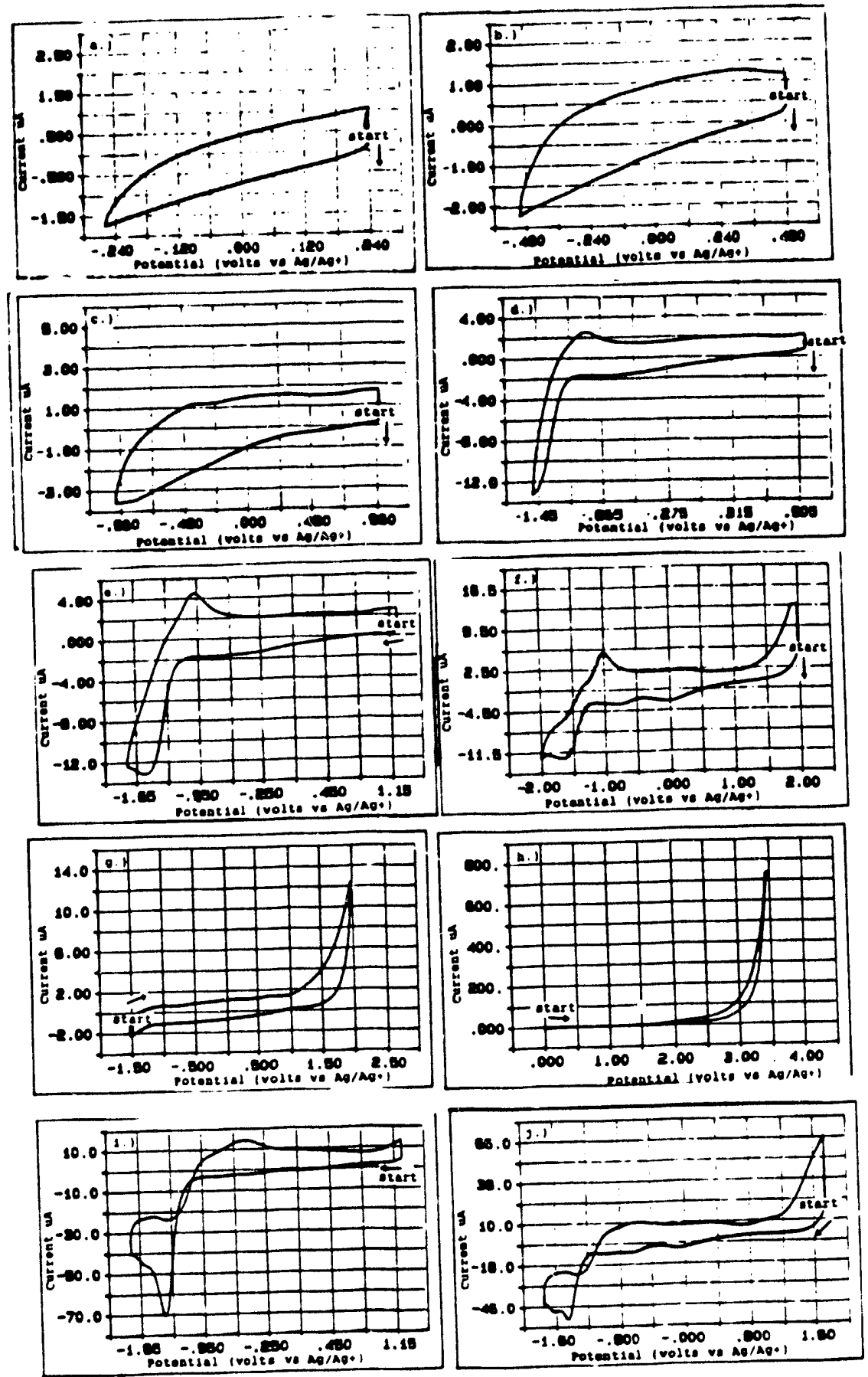
graphite but even though these two electrodes have nearly identical geometric areas, the maximum current in the case of the Pt electrode is an order of magnitude smaller than that on graphite. Also, the peak on graphite is much more reversible than that on Pt; from figure 40e the peak separation for this couple on $\mathrm{Pt}$ is $0.245 \mathrm{mv}$, whereas on graphite the peak separation is on the order of $20 \mathrm{mv}$ (see figure 39c).

Despite these differences, spiking systems of either electrode with $\mathrm{dH}_{2} \mathrm{O}$ appeared to enhance these peaks, although in the case of Pt it seems to lead to several shoulders (sef figure $40 i, j)$. Although the supporting electrolyte could be contaminated with water, (due to its hygroscopicity), and the graphite could contain additional water not removed by the vacuum treatment applied, leading to the greater current density at this electrode, the difference in the reversibility of these two sets of peaks, suggests that they may have different origins.

In addition to this comparison, in the anodic region a couple is seen on graphite at an $E_{0}$ value of approximately $1.5 \mathrm{~V}$ (see figure $39 \mathrm{~b}$ ), whereas on $P t$ a single oxidation peak is seen starting around $1.5 \mathrm{~V}$ with no corresponding reduction peak (see figure $40 \mathrm{~g}$ ). The peak seen on graphite at $1.5 \mathrm{~V}$ reaches a maximum current of $+200 \mathrm{uA}$ but the current on the $\mathrm{Pt}$ electrode at this potential is only of the order of $10.0 \mathrm{UA}$, and Pt is supposed to have a lower overpotential than the graphite electrode. 


\section{INTERCALATION}

In addition to this experimental evidence for these peaks having different origins there is a wealth of data in the literature describing reactions of graphite in AN with tetraalkylammonium salts. Graphite, and more so Highly ordered Pyrolytic Graphite; HOPG (which is close in structure to that of a single crystal), because of its layered structure is known to form inclusion or intercalation compounds (39-47). These compounds are formed by lons known as guest ions, (cations $\mathrm{M}^{+}$, or anions $X^{-}$), penetrating into the van der waals gaps between the carbon layers and enlarging the interlayer distance. The corresponding (negative or positive) charges are accepted into the host lattice (46).

$\begin{array}{lll}\mathrm{C}_{\mathrm{n}}+\mathrm{M}^{+} & +\mathrm{e}^{+} \mathrm{C}_{\mathrm{n}}^{-} \\ \mathrm{C}_{\mathrm{n}}+\mathrm{x}^{-}+\mathrm{e}^{-} & \rightarrow & \mathrm{C}_{\mathrm{n}}^{+} \mathrm{x}^{-}\end{array}$

(46).

Besides being able to sterically accept the guest ions the host lattice must be able to electronically accept the lons. Inter- 
calation reactions of the type shown above are "reversible and topotactic", the latter meaning that the reaction leads to a different interlayer distance, depending on the size of the guest ion (see Table 1)(39). Tut the carbon atom arrangement within the layers remains unchanged (46).

The stroking sequence of graphite is ( $A B A B \ldots$ )(see figure 41). In all known cases intercalation changes the stacking sequence, so that an intercalation layer of guest ions is sandwiched between equivalent carbon layers on either side ( 48 , p.36). A behavior unique to the intercalation of graphite is the formation of a regular array of unoccupied layer gaps; stage formation. This occurs only when a low concentration of the guest species is present. In compounds of stage $s$ the layers containing guest species are separated from each other by $s$ carbon layers (46). Stages from $s=1$, to 8 have been verified via $x$-ráy diffraction (46).

Tetraalkylammonium cations are known to intercalate into natural and pyrolytic graphite electrodes at approximately $-1.4 \mathrm{~V}$ (41-44, and 46) and tetrafluoroborate anions at approximately $+1.5 \mathrm{~V}(47)$. The positions and magnitudes of the peaks seen in this study are very suggastive of the intercalation of our supporting electrolyte but conclusive evidence, short of $x$-ray diffraction, is hard to come by.

The peak seen in the $P t \mathrm{cv}$ at approximateiy $1.6 \mathrm{~V}$ is probably the start of the decomposition of the acetonitrile. That 

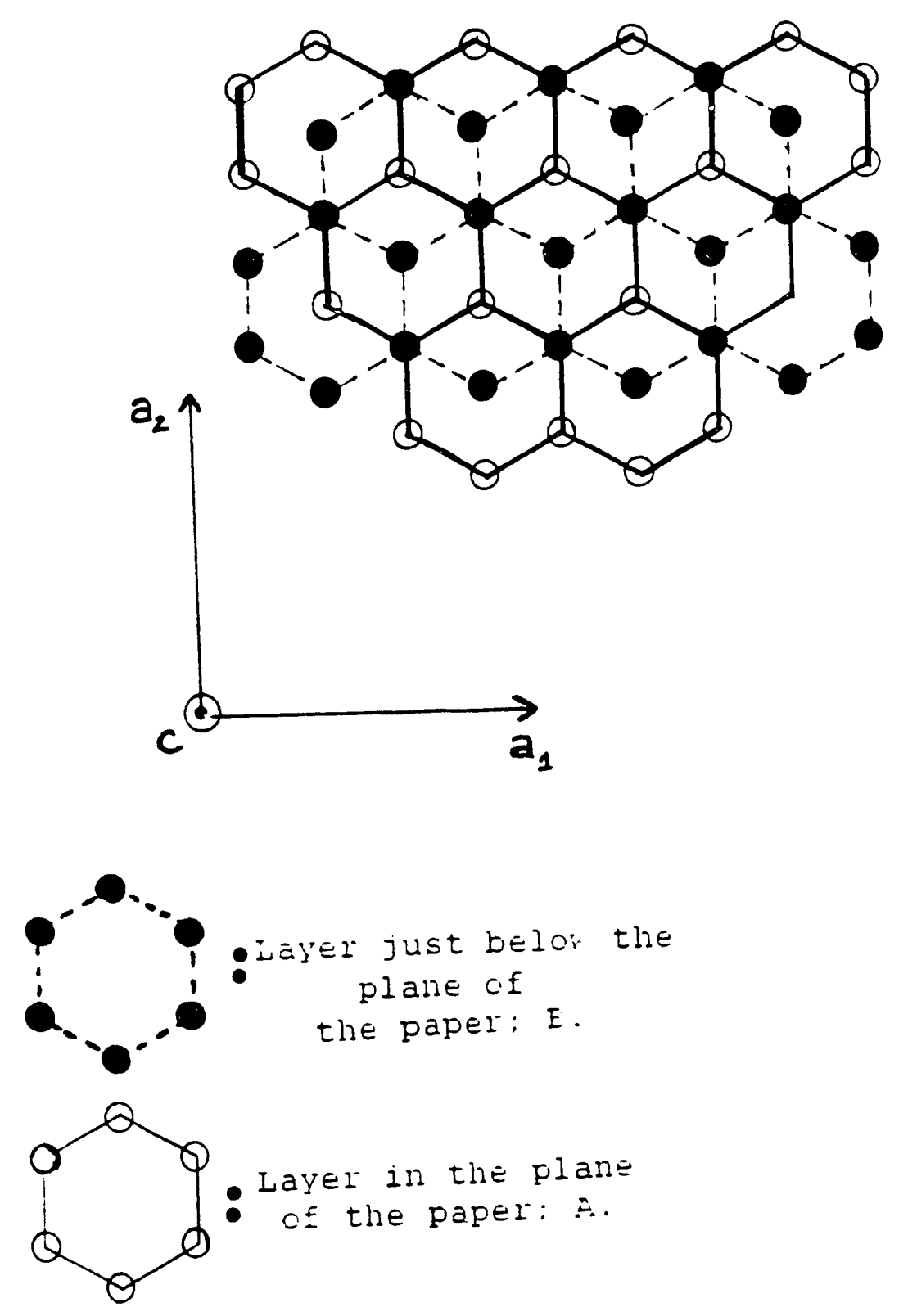

Figure 41. Stacking Sequence in P.G.

The plane of the paper is the $a_{1}, a_{2}, p l a n e$ and the $c$ axis is coming out towards you. Two graphite sheets are shown one in the plane of the paper and one immediately below it. This illustrates the ABAB... stacking sequence. 
seen at - $1.40 \mathrm{~V}$, is believed to be a contaminant although no contamination could be seen in the NMR spectrum other than water. In order to avoid, with a large margin of safety, what appears to be intercalation on $P G$, it is necessary to limit the potential range used to $-1.0 \mathrm{~V}$ to $+1.0 \mathrm{~V} \mathrm{vs} \mathrm{Ag} / \mathrm{Ag}^{+}$. However, since the ultimate goal of the larger project, of which this is a part, is to grow hydrophobic polymers on the surface of graphite and to then plate the sites in between with Pt; this as a means of hopefully, prolonging the unpoisoned life of the $P t$ as a catalyst, while also using an inexpensive substrate; the question naturally arises as to whether or not a polymer can be grown before reaching intercalation potentials?

\section{POLMERIZATION ON PYROLYTIC GRAPHITE EIECTRODES}

As a check of this, an attempt was made to grow a polymer which has been grown and carefully studied in this laboratory (49). The polymer; Poly(paraphe zylene), has been grown on a Pt slug in AN solutions. The monomer from which it is grown is paraterphenyl and it is grown by potentiostating at $1.4 \mathrm{~V} \mathrm{vs}$. $\mathrm{Ag} / \mathrm{Ag}^{+}$. In this experiment two pieces of $P G$ were used. The edge planes of both were polished as previously described. One electrode was used in a $0.1 M$ TEATFB solution and the other in a $0.1 M$ TEATFB solution, with $2 \mathrm{mM}$ paraterphenyl. Each in their respective solutions, these electrodes were exposed to 
successively greater anodic scans each starting at $0.00 \mathrm{~V}$ and scanning to an initial switching potentlal of $1.00 \mathrm{~V}$, (see figure 42a, for the supporting electrolyte solution and figure $42 \mathrm{f}$, for the monomer solution). The scan breadth was then increased by 10 $\mathrm{mv}$ and $5 \mathrm{mv}$ increments in the anodic direction for the supporting electrolyte and monomer solutions, respectively (see figure $42 \mathrm{~b}$ $e$, and $42 \mathrm{~g}-i$ ). From the cvs, it is apparent that at $1.4 \mathrm{~V}$ in the supporting electrolyte solution the current is $22 \mathrm{uA}$, whereas at this same potential in the monomer solution the current is almost $100 \mathrm{uA}$. It therefore appears possible to grow poly(paraphenylene on edge plane, PG.

\section{OPTIMUY ELECTRODE ACTIVITY}

The next question which needed to be answered was which pretreatment method provided the most active surface? In order to understand what is meant by active surface, a definition of active site is needed. An active site is a site where electron transfer can occur. By most active surface it is meant, the surface that has the greatest number of active sites per unit effective area. A variety of pretreatment procedures exist: electrochemical oxidation (50), treatment with an oxygen radio frequency plasma (34, 51), Vacuum Heat Treatment at $600^{\circ} \mathrm{C}$ and $10^{-B}$ torr (10), and in situ laser activation (52). Some of these

methods increase the presence of surface oxides $(34,50,51)$, 

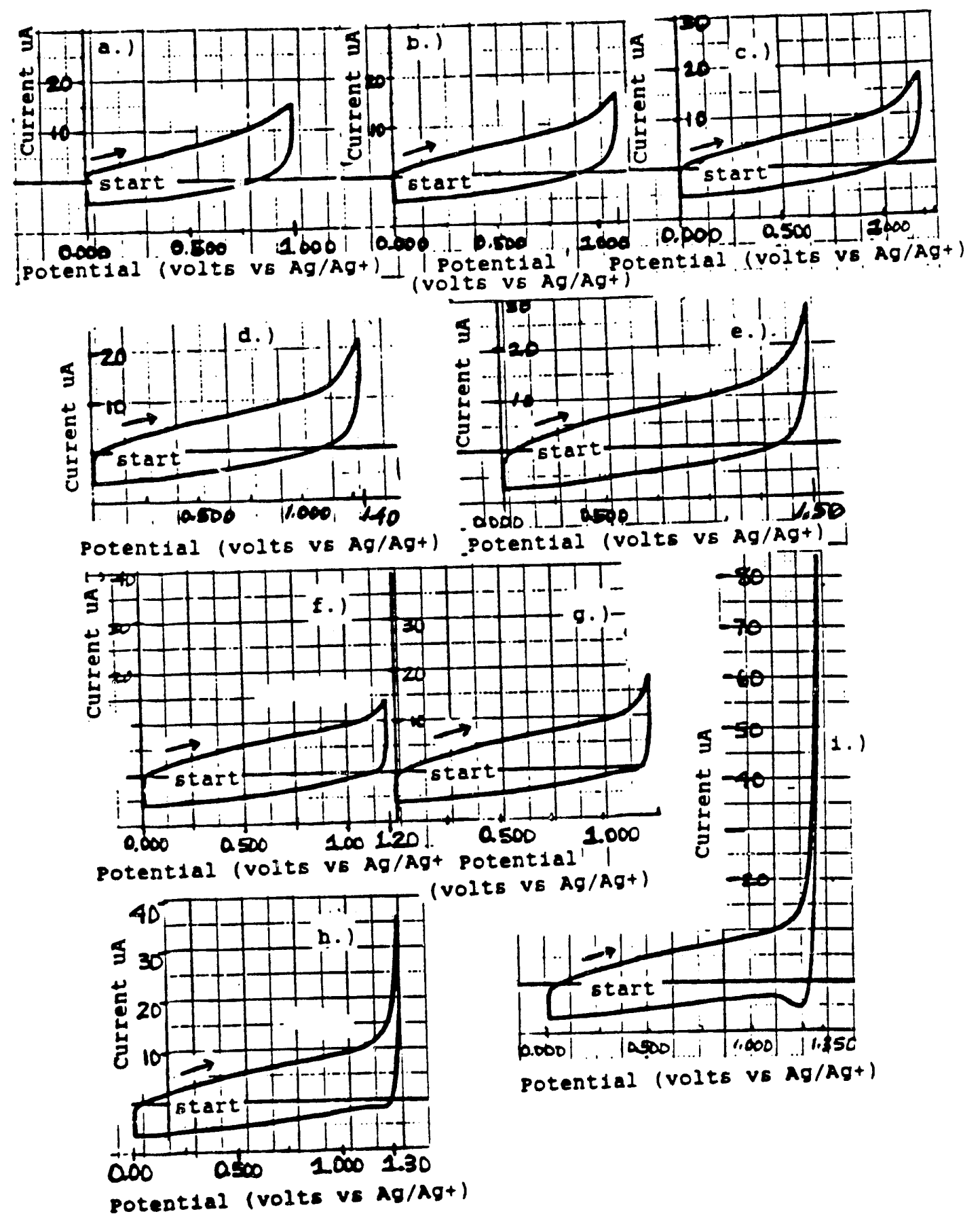

Figure 42. Mononer vs Supporting Electrolyte Solution

(a) - (e) are cvs for the supporting electrolyte solution and (f) - (i) are cvs for the monomer solution. All are run at $50 \mathrm{mv} / \mathrm{sec}$. 


\begin{abstract}
some remove surface oxides $(10,52)$ but all are believed to increase the activity of the surface. However, in order to measure the surface activity, a prerequisite is to know the true area of the electrode and determining this led to what became the primary area of study in this thesis; capacitance measurements.
\end{abstract}




\section{APPENDIX III:}

\section{AREA DETERHINATION - DIFFUSION DEPENDENT TECHNIQUES AN ATTETPT AT NONAQUBOUS CHRONOANTERONETRY:}

Some of the most frequently employed electrochemical procedures for determining electrode area are the related techniques: chronoamperometry, chronocoulometry, and chronopotentiometry. All of these techniques rely on the establishment of linear diffusion conditions for measuring the area. Here an attempt was made to use chronoamperometry to measure the area of the edge plane, PG electrodes in AN solutions.

In chronoamperometry, the working electrode is immersed in a solution, (usually aqueous), containing an excess of supporting electrolyte, and an oxidizable or reducible analyte at $\mathrm{mM}$ concentrations. With working electrode, reference, and auxiliary electrodes set up, all are hooked up to a potentiostat. The cell dimensions must be large relative to the working electrode dimensions and the cell must be free of mechanical and thermal disturbances. The input signal applied to the electrochemical cell is a square wave voltage signal, see figure 43 . If the analyte, a neutral species, is to be reduced, then the applied potential $E_{s}$ should be $50-100 \mathrm{mv}$ negative of its standard 


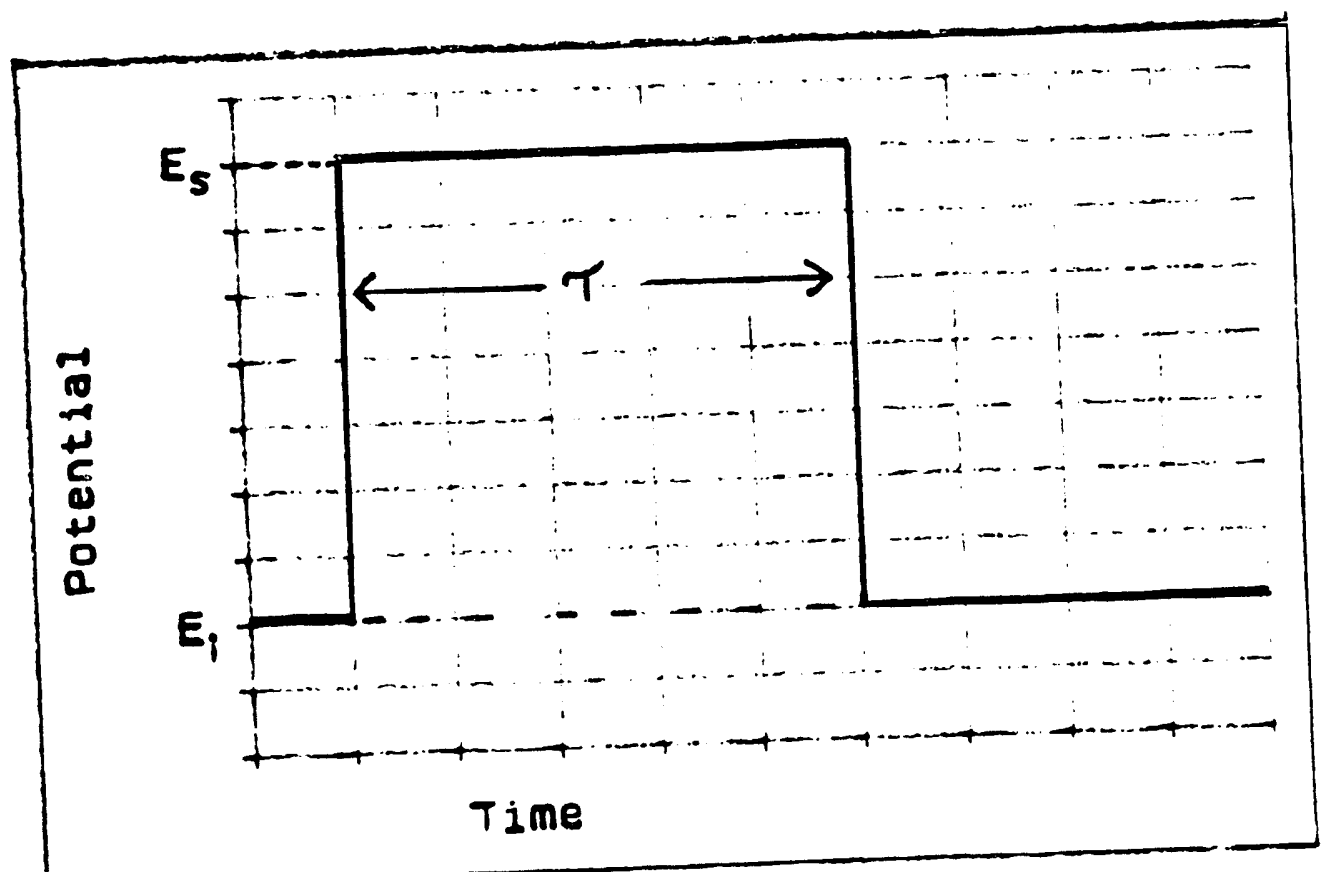

Figure 43. Chronoamperometry Input Waveform

From an initial potential; $E_{i}$, the potential is stepped to $E_{S}$, for time; $\tau$. 
reduction potential and if it is to be oxidized, then $E_{s}$ should be 50-100 mv positive of its standard oxidation potential.

In an aqueous solution, the potential must not be so anodic as to cause $\mathrm{O}_{2}(\mathrm{~g})$ evolution, or so cathodic as to cause $\mathrm{H}_{2}(g)$ evolution but in a nonaqueous solution, this is not a problem. The only potential limit concerns, in the system investigated here, are the intercalation potentials (see appendix 2). According to Adams (53), another concern in nonaqueous solutions may be the insufficiently high viscosities. This will be discussed further later on.

The voltage signal, shown in figure 43, is stepped from a potential where no faradaic current flows to a potential where the concentration of the analyte at the electrode surface goes to zero, due to the complete oxidation or reduction of the analyte in this region. A zero concentration of the analyte at the surface of the electrode is important to establish the diffusion limited current. With the application of the input voltage signal, the current output is recorded as a function of time, which is where chronoamperometry gets its name.

The equation which defines the instantaneous current under conditions of linear diffusion is known as the Cottrell equation; equation 9 .

$$
i_{t}=\left[\mathrm{nFAD}{ }^{1 / 2} C_{b}\right] /\left[\pi^{1 / 2} t^{1 / 2}\right] \text { equation } 9(53, \text { p.50) }
$$


In this equation, $C_{b}$ is the concentration of the neutral analyte in the bulk of the solution, $D$ is the diffusion coefficient of the unreacted bulk analyte, $A$ is the area of the electrode, $n$ the number of electrons involved in the electron transfer reaction, $F$ is the faraday constant, and $\pi$ has its usual meaning. This equation assumes that the concentration of the analyte is zero at the surface of the electrode, and it assumes linear diffusion is the only form of mass transport. If $n$, and $D$, are known, then the only unknown is the area.

At least two independent authors have used ferrocene as the analyte in their chronoamperometric measurements. Sharp (54) and Bond; Henderson; Mann; Mann; Thormann; and Zoski (55) used ferrocene as an analyte in $0.1 \mathrm{M}$ tetraethylammonium perchlorate (TEAP); AN solutions, to determine the area of their Pt electrodes. Here, an attempt was made to likewise use ferrocene in AN; TEAP solutions, but to measure edge plane, PG electrode area.

In these experiments a $1 \mathrm{mM}$ ferrocene; 0.1 MEAP; AN solution was prepared (ferrocene 988 pure, courtesy of the Koch research group at SUNY Stony Brook Dept. of Chemistry), TEAP purchased from Eastman Kodak Organic Chemicals. The acetonitrile for this solution was purified in the usual way (see experimental section). Electrodes were polished as described in the experimental section, except where specified differently, and were then dried under vacuum as described in the experimental 
section. After the solution was added to the cell and the cell assembled, $\mathrm{N}_{2}(\mathrm{~g})$ was bubbled through the solution at a high rate in the cell for 8 minutes; to get rid of $\mathrm{O}_{2}(g)$, and then at a much lower rate into the empty space above the solution level in the cell; to keep $\mathrm{O}_{2}(\mathrm{~g})$ out.

It was important to bubble above the solution level and at a much slower rate because this technique requires solution quiescence in order for the necessary diffusion conditions to be met. After 5 minutes of bubbling $N_{2}(g)$ in this arrangemeni, the strip chart recorder mode of Asystant+ Computer Assisted Data Acquisition Software was started. A few seconds were allowed to pass to establish a base line of zero current, and then the potentiostat was turned on at a potential of $0.400 \mathrm{~V}$ v $\mathrm{Ag} / \mathrm{Ag}^{+}$, which was the value for $E_{S}$. The time;,$E_{S}$ was applied for was 1 minute and the decaying current was recorded as a function of time. The potentiostat used for this experiment was the EG\&G potentiostat and the current range setting of this instrument was 100 uA full scale.

In early experiments, before the ferrocene; $F e C P_{2}$, solution was added a run was made in a blank, containing only $0.1 \mathrm{M}$ TEAP; AN, to see the level of the background current. The procedure described above was also performed for these solutions. If the background current was significant, it was to be subtracted off of the current measured in the $\mathrm{FeCP}_{2}$ solutions but the background current was found to be insignificant. 
From the current decay plot, values of $1 t^{1 / 2}$ can be obtained and since the concentration of the ferrocene in the bulk is known to be $1 \mathrm{mM}$ and the diffusion coefficient of the ferrocene was found in the literature to be $2.3 \times 10^{-1} \mathrm{~cm}^{2} \mathrm{~s}^{-1}(55)$; the area is the only unknown in the equation.

A systematic study was conducted of the calculated area of electrodes prepared in two ways. For one type of preparation the electrodes received the polishing pretreatment described in the experimental section, for the other set they received only the first step of the polishing procedure described in the experimental section, (that with the 1-2 um $d p$ ). This should have created electrodes with different true areas; the ones having been polished with only the $1-2$ um dp having a greater surface roughness.

The resuils from these experiments are shown in figure 44 , which is a histogram using area ranges. Each hand drawn rectangle is an occurrence of a measured electrode area within the specified area range. The rectangles filled with circles are for the partially polished electrodes or roughened electrodes and the rectangles filled with cross hatch are for electrodes completely polished. The area of the electrode calculated from just the geometric dimensions of the electrode is $0.08 \mathrm{~cm}^{2}$. Only two of the electrodes gave a value close to within $\pm 10 \%$ of this; the rest all had greater values. No correlation was found between the degree of polishing and the measured area. Why wasn't it found? 


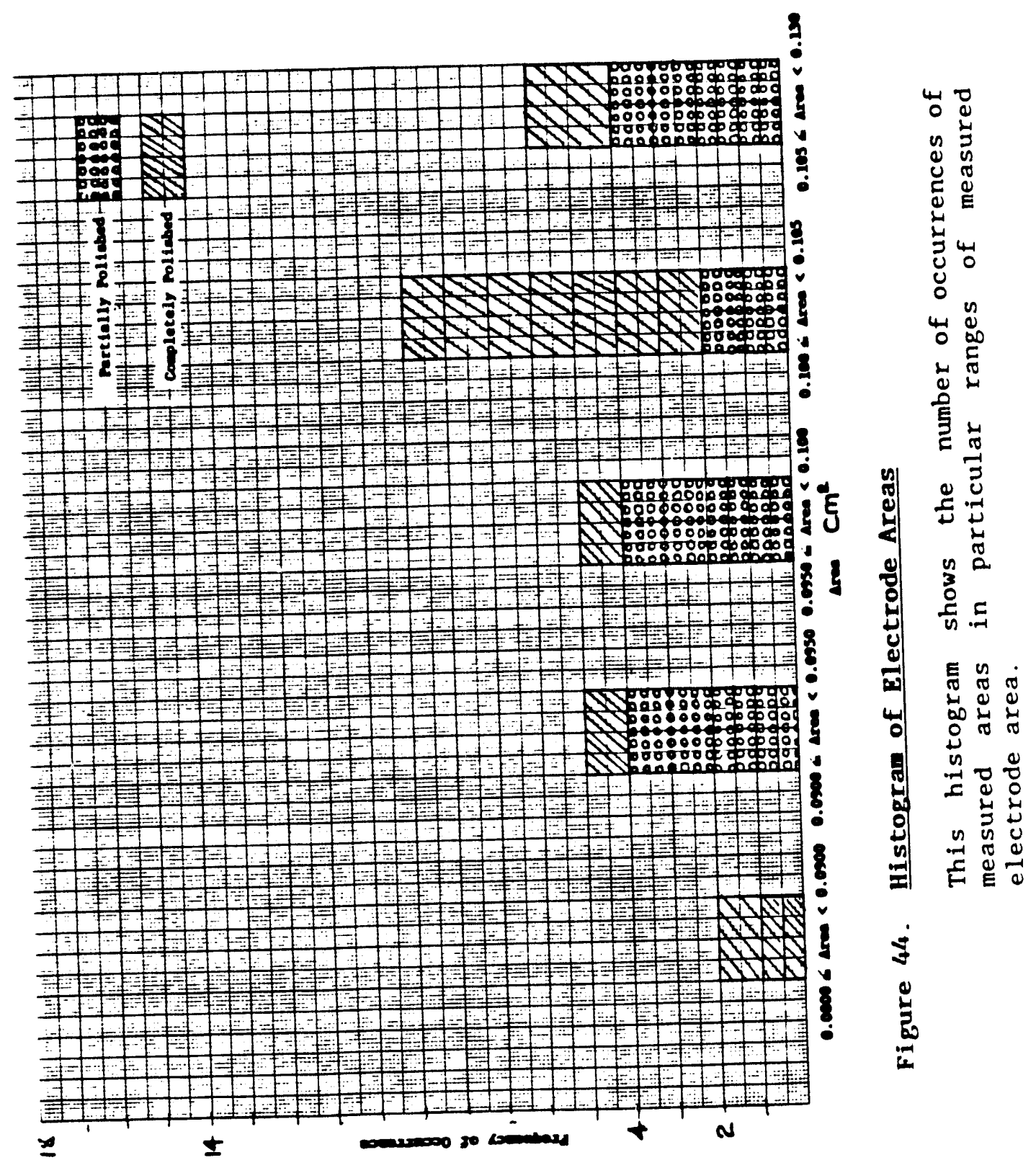




\section{UHICH AREA IS A?}

Which area does chronoamperometric measurements, the $A$ in equation 9 , represent the true area or the geometric area? Several independent authors of books and scientific articles have made statements which seem to suggest that $A$ is the true area: "The alm of this study", (chronoamperometry study), "was to establish an appropriate procedure for the determination of the effective or electrochemically active area of graphite electrodes for use in the investigation of polymer films as electrode coatings." Coury and Heineman (56, p.328); and as Adams (53, p.50) says: "hence the electrochemical area of an electrode can be determined provided $n$ and $D$ are known." It is unclear what Adams meant by "electrochemical area", although it is usually a term associated with the true area. Adams may have meant the geometric area determined electrochemically. Heineman, however, in the first quotation, seems to be referring to the true area.

In spite of confusing statements like these, an analysis of the conditions under which equation 9 is applicable leads one to conclude that $A$ in equation 9 is not the true area but the geometric area determined electrochemically. The geometric area determined electrochemically could differ from the geometric area measured from the dimensions of the electrode since the dimensions are not perfect. The analysis which supports this conclusion: that chronoamperometry and all diffusion dependent 
techniques measure the geometric area electrochemically, is that the condition $C_{e} \rightarrow 0$ must be met in order for the techniques to be used, where $C_{e}$ is the concentration of the analyte at the surface of the electrode.

Once this condition is met, the measured current is thereby dependent on the diffusion of the unreacted analyte through a projection of the electrodes surface some distance $x$ into the bulk solution. This projection knows nothing of the surface roughness and therefore the measured diffusion dependent current knows nothing of the surface roughness.

Statements made by independent authors which support this interpretation are: "The geomerric area exposed to the solution was $0.084 \mathrm{~cm}^{2} \pm 0.014 \mathrm{~cm}^{2}$ (calculated from chronocoulometry)" Kuwana (10, p. 2760); "The roughness factor is this effective area divided by the geometric cross sectional area, the latter calculated from the dimensions of the electrode or from results of an experiment measuring the diffusion limited current." Kuwana (10. p.2759); "The area of a polished electrode (taken to be the projected or geometric area in most voltammetric experiments at times greater than 1 sec) usually is measured directly or electrochemically. - In practice two methods are used for stationary planar electrodes in quiescent solution - chronoamperometry and chronocoulometry" Sawyer and Roberts (7, p.75); "Chrciocoulometry was used to evaluate electrodes in terms of 
their effective areas (or "projected geometric areas") by employing two different methods." Coury and Heineman (57, p.554).

An important fact further supporting this interpretation is that the thickness of the depletion region around the electrode surface (the diffusion layer thickness) for electrolysis times that are greater than one second ( 7, p.74) is substantially larger than the characteristic dimensions of the surface roughness and therefore the projection of the electrode into the solution knows nothing of the surface roughness.

\section{IS THE VISCOSITY OF ACETONITRILE A PROBLEA?}

According to Adams (53), chronoamperometric measurements can not be made in nonaqueous solvents. This is attributed to the low viscosity of these solvents. In solvents of low viscosity Adams (53) believes that nonlinear diffusion and other means of mass transport set in early. The result of this is an it ${ }^{1 / 2}$ product, (it ${ }^{1 / 2}$ is supposed to be constant, see equation 9), which "increases very rapidly during the entire course of the potential measurement." (53, p.21) see figure 45. If a plot of it ${ }^{1 / 2}$ vs time is made for data from this work (see figure 46), the increase in $i t^{3 / 2}$ with time that Adams observed is not seen, and therefore, the viscosity of the solution does not appear to be a problem. In this figure the sharp rise seen on the left is when the potential was first applied and is the depletion of the 


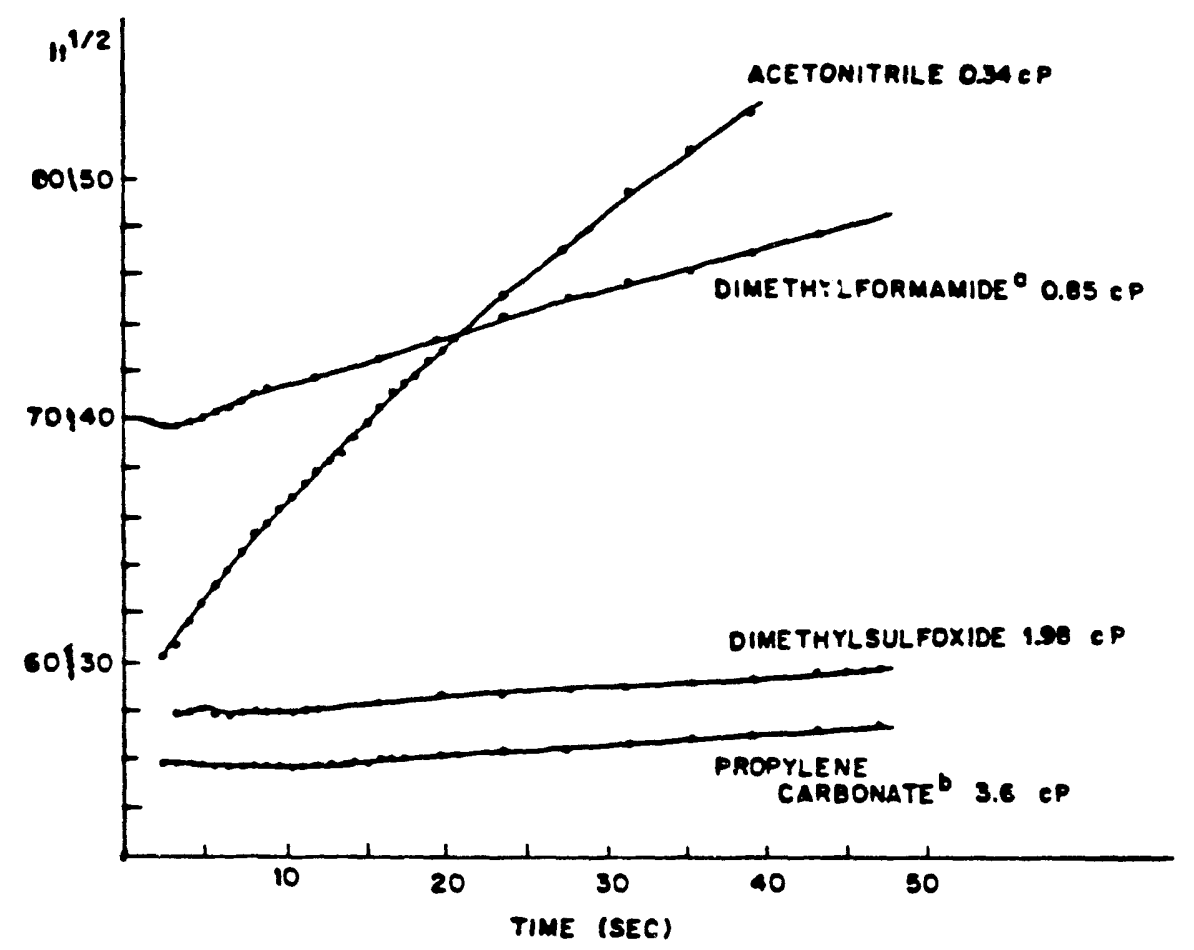

Variation of $i t^{3 / 2}$ with solvent viscosity for some nonaqueous systems. Viscosities from standard tables except as noted: a, viscosity measured with $0.1 M$ tetraethylammonium perchlorate as supporting electrolyte; $b$, viscosity measured with $0.5 M$ tetraethylammonium perchlorate as supporting electrolyte. Figure 45. Viscosity Dependence of it ${ }^{1 / 2}$ for different Nou-

Ideally, it ${ }^{1 / 2}$ should be constant. Here, it is plotted as a function of time for solvents which supposedly do not have sufficiently high viscosities. The viscosities and the name of the solvent are written to the right hand side of each of the corresponding curves. Reprinted from Adams, R.N. Electrochemistry at Solid Electrodes; Marcel Dekker: NY, 1965; $\mathrm{p} 45$, by Courtesy of Marcel Dekker Inc. 


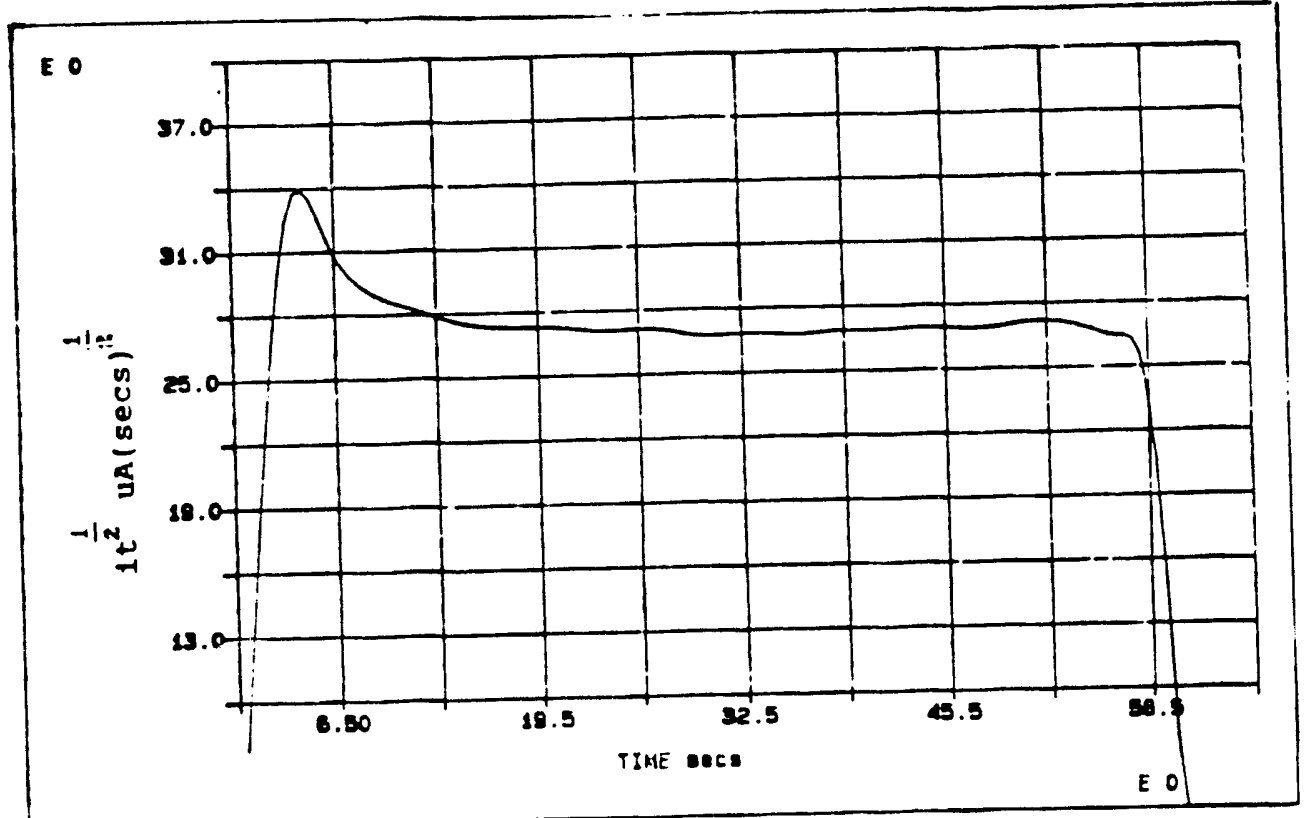

Figure 46. Behavior of it ${ }^{1 / 2}$ for Acetonitrile found here This figure shows it ${ }^{1 / 2}$ vs time for experiments conducted in this work. 
solution of ferrocene at the surface of the electrode, after which, diffusion control sets in. The sharp fall on the right is at time; $T$, when the potential is returned to its initial value. So, the viscosity is not a problem.

\section{IS FERROCENE A REVERSIBLB COUPLE?}

Besides a purported difficulty with AN, the electrochemistry of ferrocene has recently been shrouded in controversy. The controversy stems from inconsistencies in reported values of rate constants by a number of independent investigators (54).

Even for measurements made in the same solvent and supporting electrolyte, made by the same measurement method, rate constants have been reported which differ by as much as a factor of five (54). In addition to this, disagreement has arisen over whether ferrocene is a model of a reversible electrode process, at Pt and C electrodes, or as Bond (55) reports, that Kadish has concluded ferrocene can be used as a model of a quasireversible electrode process.

In addition to these claims J.W. Pons and S. Pons have cast more shadows over this redox couple by claiming to have formed a film on a Pt electrode with a ferrocene complex, salt, or polymeric layer (60). The experimental conditions under which this film was formed are the following: 1.) the solution was 10 
$m$ M ferrocene in 0.1 M TBATFB (tetrabutylammonium tetrafluoroborate) in acetonitrile; 2.) the working electrode was a Pt wire; 3.) the potential was scanned from $-1.00 \mathrm{~V}$ to $0.750 \mathrm{~V}$ and back vs. a Ag/ $\mathrm{Ag}^{+}\left(0.01 \mathrm{M} \mathrm{AgNO}_{3}\right.$ in $\left.0.1 \mathrm{M} \mathrm{TBATFB;AN}\right)$ reference electrode; 4.) the scan rate was $50 \mathrm{mv} / \mathrm{sec} ; 5$. ) once the original voltage of $-1.00 \mathrm{~V}$ was reestablished the electrode was potentiostated at this voltage for a few minutes until the scan was repeated (58).

This experiment was repeated here, except with the following differences: instead of $0.1 \mathrm{M}$ TBATFB, we used $0.1 \mathrm{M}$ TEATFB; the working electrode was a edge plane, $P G$ electrode, not a $P t$ electrode, (since we wanted to see if this behavior could be affecting our measurements and if the two electrodes could behave differently); and the reference electrode was a $\mathrm{Ag} / \mathrm{Ag}^{+}$ion electrode $0.1 \mathrm{M} \mathrm{AgNO}_{3} ; \mathrm{AN}$. Film formation occurred and the cyclic voltammograms look similar to those seen by Pons (58) (see figure. $47 \mathrm{a}$ and $\mathrm{b})$. Like Pons, the potential was scanned from - $1.0 \mathrm{~V}$ to $+0.75 \mathrm{~V}$ at $50 \mathrm{mv} / \mathrm{sec}$. The $\mathrm{cv}$ in $47 \mathrm{a}$ with the largest current is the first scan and with each successive scan the peak current became smaller, for both the oxidation; the peak with positive current, and the reduction; the peak with negative current. The peak with the smallest current in figure $47 \mathrm{a}$ is the $15^{\text {th }} \mathrm{scan}$. In figure $47 \mathrm{~b}$ are shown the $16^{\text {th }}$ through the $18^{\text {th }}$ scans, again the peak current dropping with increasing scan number; here a second couple with much greater peak separation is visible. 

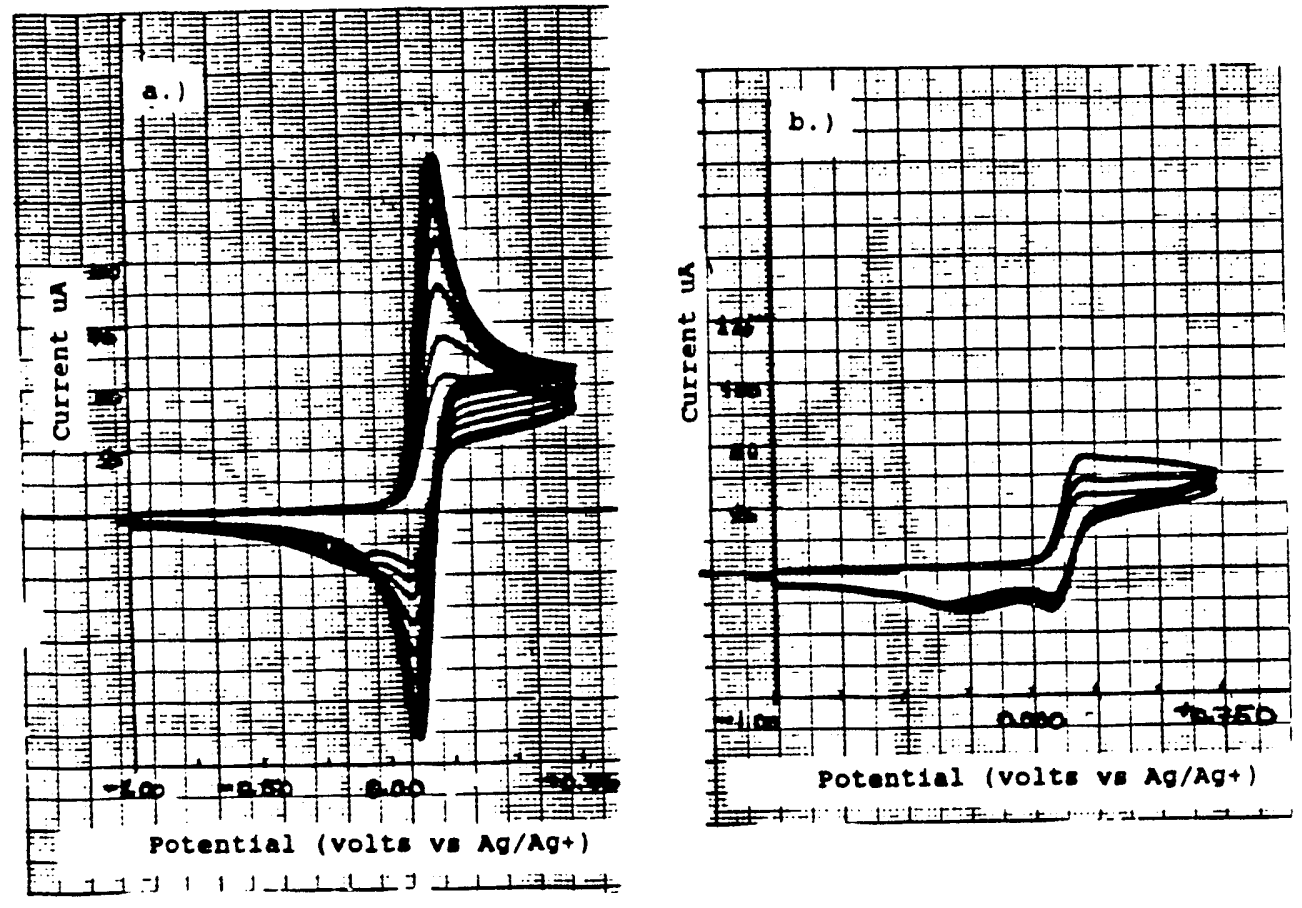

Figure 47. Ferrocene Filn Formation

(a) Scans were run from $-1.0 \mathrm{~V}$ to $+0.75 \mathrm{~V}$ at 50 $\mathrm{mv} / \mathrm{sec}$. The cv here with the largest current is the first scan and the one with the smallest current is the 15th. (b) 16 th -18 th scans. 
The fact that I.U.P.A.C. In 1983 (61) made recommendations for the use of ferrocene as a reference redox couple, for establishing a basis for reporting nonaqueous reference electrode potentials, further confuses this situation. Reference redox systems are supposed to be ideal redox couples which have nearly solvent independent redox potentials. However, if Kadish reports that the system is quasireversible; if Pons reports film formation and at best quasireversible behavior; how can it be used as a reference redox system and more importantly, here, how can it be used as an analyte in chronoamperometry?

However, I.U.P.A.C. did not recommend that such a large potential range be used when checking for the redox potential, nor did they recommend such a high concentration. If a narrow potential range is used and if a dilute solution is used, i.e. approximately $1 \mathrm{mM}$, film formation is not seen in this work with repeated scans. As regards the claims of inconsistent electrochemical rate constants and quasireversible behavior, another author Bond (55), claims that ferrocene is an excellent model of a reversible system in $\mathrm{AN}$ and Kapoor and Hao (60) have results which seem to suggest a concentration dependence over the ranges used in these experiments, which might explain some of the behavior seen.

Kapoor and Hao (60) performed studies of ferrocene at different concentrations, from $5 \times 10^{-4} M$ to $5 \times 10^{-3} M$, that indicate that ferrocene approaches thermodynamic reversibility 
only within a narrow concentration range $(60)$. At $5.0 \times 10^{-4} \mathrm{M}$ Ferrocene, at a glassy carbon electrode, in $0.1 \mathrm{M}$ TEAP in AN, with a $0.01 \mathrm{M} \mathrm{Ag} / \mathrm{AgClO}$, reference electrode, and at a scan rate of $100 \mathrm{mv} / \mathrm{sec}$, they saw the position of the potential of the cathodic current peak at; $E_{p c}-0.045 \mathrm{~V}$, and the position of the potential of the anodic current peak at; $\mathrm{E}_{\mathrm{pa}}=0.110 \mathrm{~V}$, and therefore a peak separation, $\Delta E_{p}=0.065 \mathrm{~V}$. At $5 \times 10^{-3} \mathrm{MFeCP}_{2}$, under otherwise identical conditions, they saw $E_{p c}-0.028 \mathrm{~V}, E_{p a}=$ $0.145 \mathrm{~V}$, and $\Delta E_{p}-0.117 \mathrm{~V}$. Therefore it seems that the couple is reversible only at low concentrations.

By the results of these experiments, and the literature research, it was concluded that chronoamperometry could be used in acetonitrile solutions and with ferrocene as the analyte but that the area measured was the geometric area. However, one method for measuring the true area which is nondestructive and can be used in the environment of interest, (in situ), is based on measuring the capacitance of the electrode. This possibility was the motivation, and ambition behind this study of the capacitance of the edge plane of PG electrodes in AN solutions. 


\section{Appendix IV:}

This appendix illustrates the dependence of the Open Circuit Potential; OCP, on the various electrode pretreatment procedures employed. Figure 48 is a histogram of the frequency of occurrence of set ranges of OCPs for electrodes receiving as the final pretreatment procedure: Radio Frequency Plasma Treatment, Oven Drying, Vacuum Drying, and Vacuum Heat Treatment.

As the pretreatment procedure is changed in this order the measured OCPs become progressively less positive and then more negative. Radio Frequency Plasma Treated electrodes showed the most positive OCPs and Vacuum Heat Treated electrodes the most negative. 


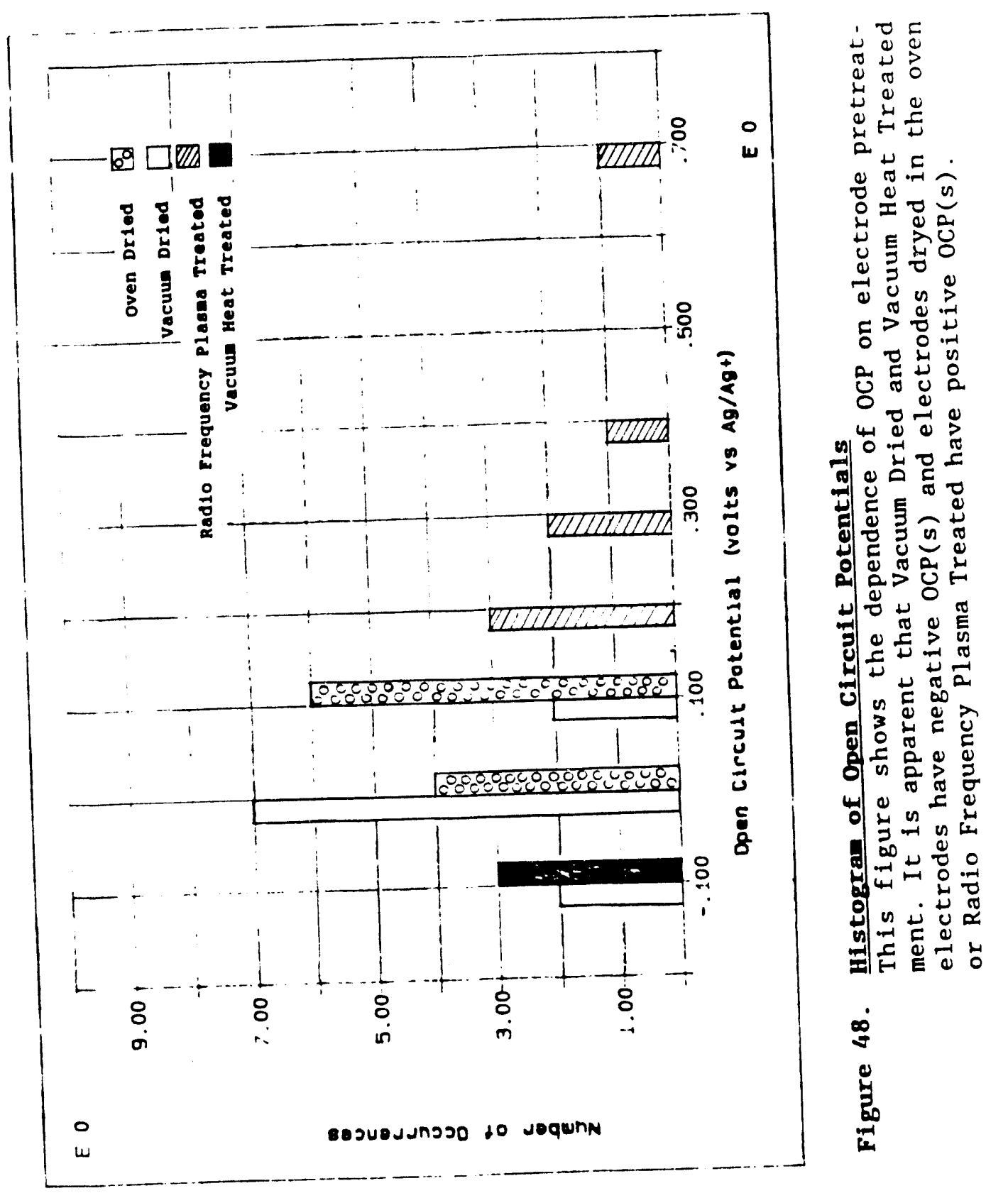




\section{Appendix V}

This appendix shows the derivation of the function used to fit the $c / d$ curves. The input signal and equivalent circuit are shown on the following page, in figures $49 a$ and $b$, respectively.

$$
\begin{aligned}
& e_{i n}=e_{0}+\nu t-e_{R}+e_{C} \\
& e_{i n}: \text { applied potential; input potential. } \\
& e_{0}: \text { initial potential; before scanning. } \\
& e_{C}: \text { potential drop across the capacitor. } \\
& e_{R}: \text { potential drop across the resistor. } \\
& t: \text { time. } \\
& \nu: \text { scan rate of potential sweep. }
\end{aligned}
$$

Taking the derivative with respect to time yields:

$$
\begin{aligned}
& \frac{d e}{d t} i n=\nu-\frac{d e}{d t} R+\frac{d e}{d t} C \\
& \text { Since, } C=\frac{1}{d e} C \text {, and therefore } \frac{d e}{d t} C=\frac{i}{d} C \text {, then: } \\
& \nu=\frac{d e}{d t} R+\frac{i}{C} C=R \frac{d i}{d t}+\frac{i}{C} C
\end{aligned}
$$

Because this is a series circuit the instantaneous current flowing through the resistor and the capacitor are equal and therefore the subscript $C$ is dropped from ${ }^{{ }_{C}}$ and $i$ henceforth always refers to the instantaneous current. 

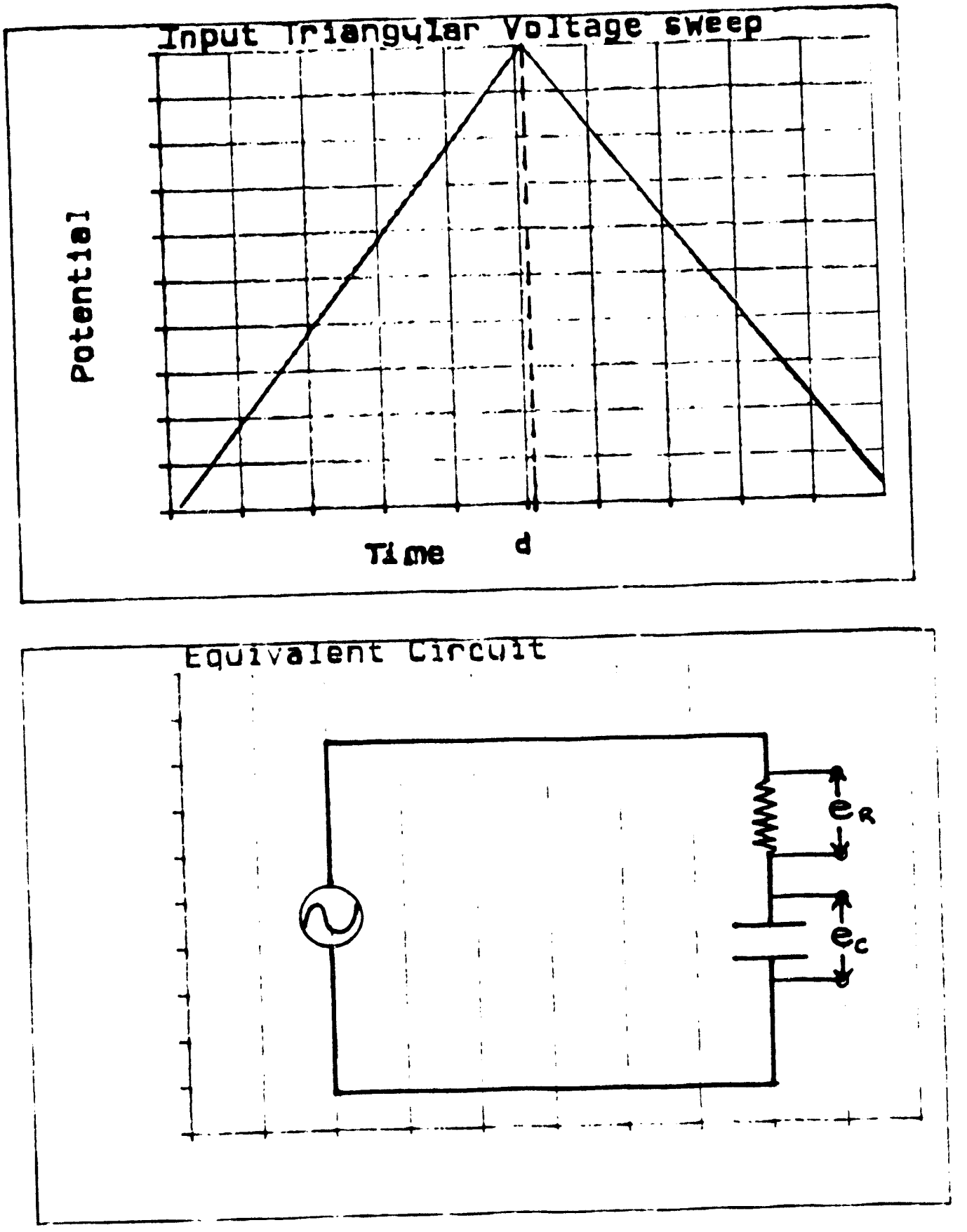

Figure 49. Imput Signal and Equivalent Circuit

(a) This figure shows the input triangular voltage signal, and $d$ is the time of the reversal of the scan direction. (b) This figure shows the assumed equivalent circuit. 
152

$$
\begin{aligned}
& \frac{1}{C}-\nu--R \frac{d i}{d t} \\
& 1-\nu C--R C \frac{d i}{d t} \\
& (1-\nu C) d t=-R C d i \\
& -\frac{d t}{R C}=\frac{d i}{(1-\nu C)} \\
& -\frac{d t}{R C}-\frac{d u}{u} \quad \begin{array}{l}
u-1-\nu C \\
d u-d i \\
u(@ t-0)-\nu C \\
u(@ t-t)-i-\nu C
\end{array}
\end{aligned}
$$

For $t<d, \nu>0$ and since $\nu C \geq 1, u-1-\nu C$ is negative. Because of this, set $u-\cdots(i-\nu C)$ and du - -di.

$$
\begin{aligned}
& -\frac{d t}{R C}=\frac{d u}{u} \\
& -\int_{0}^{t} \frac{d t}{R C}=\int_{\nu C}^{\nu C-i} \frac{d u}{u}-\frac{-t}{R C} 0-\ln u \nu C-i \\
& \frac{-t}{R C}=\ln \frac{(\nu C-i)}{\nu C} \\
& e^{-t / R C}=\frac{\nu C-i}{\nu C} \\
& \nu C e^{-t / R C}=\frac{\nu C-1}{i}=\nu C-\nu C e^{-t / R C} \\
& i=\nu C\left(1-e^{-t / R C}\right)
\end{aligned}
$$


For $t>d, \nu<0$ and since $\nu C \geq i, u-i-\nu C$ is positive and du - di, making the above integration for this case even more straight forward. The sime solution is obtained:

$$
i=\nu C\left(1-e^{-t / R C}\right) \text {. }
$$


XII APPENDIX VI: 
Program CAPFB for one independent variable: By o modified

simplex iteration the program minimizes the "error" defined as

Error = sum over exptl pts $\left[y(\exp )-f\left(c a l^{\prime} d\right)\right] * 2$

where $f$ is $y$ (linear or non-linear) function defined in the

"errorf" subroutine. (Also found in the PLOTDAT subroutine.)

* Sizes of dimensioned variables **

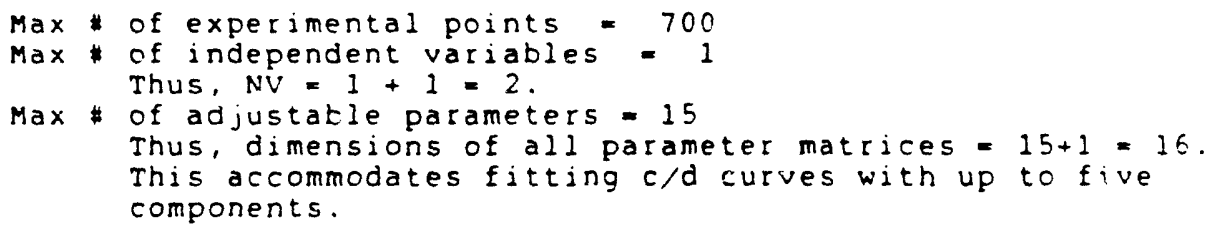

The file should be there before starting the program.

(2) All other inputs are frum keyboard. Answer the prompts by typing appropiate numbers(s) and hitting (cr). The program will first ask for 5 items of information as follows.

(2a) Number of parameters (np). The largest number allowed is 15, which corresponds a c/d curve with 5 components.

(2b) The scan rate in volts per second.

(2c) Initial estimates of parameter values, 3 numbers fer line, separated by one or more blanks. Any format (e.g., F, E, D, G) is accepted. The program will keef asking for more data until "np" numbers have been read. The program reads in the value of "a" first, followed by the resistance in ohms and the capacitance in $F$ for this component term of the series of exponentials.

(2d) maxent: Maximum number of iterations you will allow for this run. It can be any positive integer (e.g.. 1000 ) but be conservative: You can always augment this later on by means of "more" (cf: Input (3c)). 
(2e) Termination criterion (errmin), the relative spread among the $(n p+1)$ sets of parameters in the last iteration. (See ( $2 \mathrm{f}$ ) below for significance of $n p+1$.

errmin> (1worst error of last 3 ) - (best of last 3 ))

The program stops when the RHS becomes smaller than errmin. Example: errmin $=1.0003$

(2f) Initial offset factor (factor): The simplex procedure finds a best set of (np) parameters by manipulating (np $+1)$ sets of (np) parameters at any phase of the iterative process. In the beginning, the program will create np sets out of the initial-guess set by multiplying one of the parameters by thic factor, one at a time. These np sets and the initial guess set constitute the first "simplex." Use a large factor (e.g., 1.1) for a coarse search and a smaller factor (e.g., 1.001 ) for a finer fit.

(3) Depending on how the iterations are converging, the program will ask for additional information, the major ones being as follow's.

(3a) Questions which allow plot of the error vs one of the parameters. Just follow on-screen instructions.

(3t) scale = 1 terminates the current run.

$0<$ scale $<1$ causes a detailed examination of a smaller segion around the current best set of parameters.

scale $=0$ causes no change of scale and the

scale $<0$ control goes to the "MORE" question. expands the search region around the current best.

Essentally, the range of parameters around the current best set is multiplied by 11 - (scale)). Thus: scale $=0.9$ contracts the search range by a factor of 10 , and scale $=-9$ expands the range by a fastor of 10 .

The errmin value had better be reset when the "scale" is changed. Example: If scale=0. is set, also set errmin to 10 times smaller than before.

(3c) more $=4$ continues current search using new values for maxent and errmin. Follow the on-screen instructions to input new values.

more $=3$ lets you manually reset parameter value!s 1 Those you do not specify here will remain at their values in the last best set. The program will then let you specify maxcnt, errmin, and factor. With the new "factor" and the new "best set". the program will produce a new "simplex".

more $=2$ lets you freeze specified parameter(s) at the value(s) you want. The program will then let you specify a new maxcnt, errmin, and factor. Then it will use the "reduced" parameter array and the new "factor" to produce a new "simplex". 


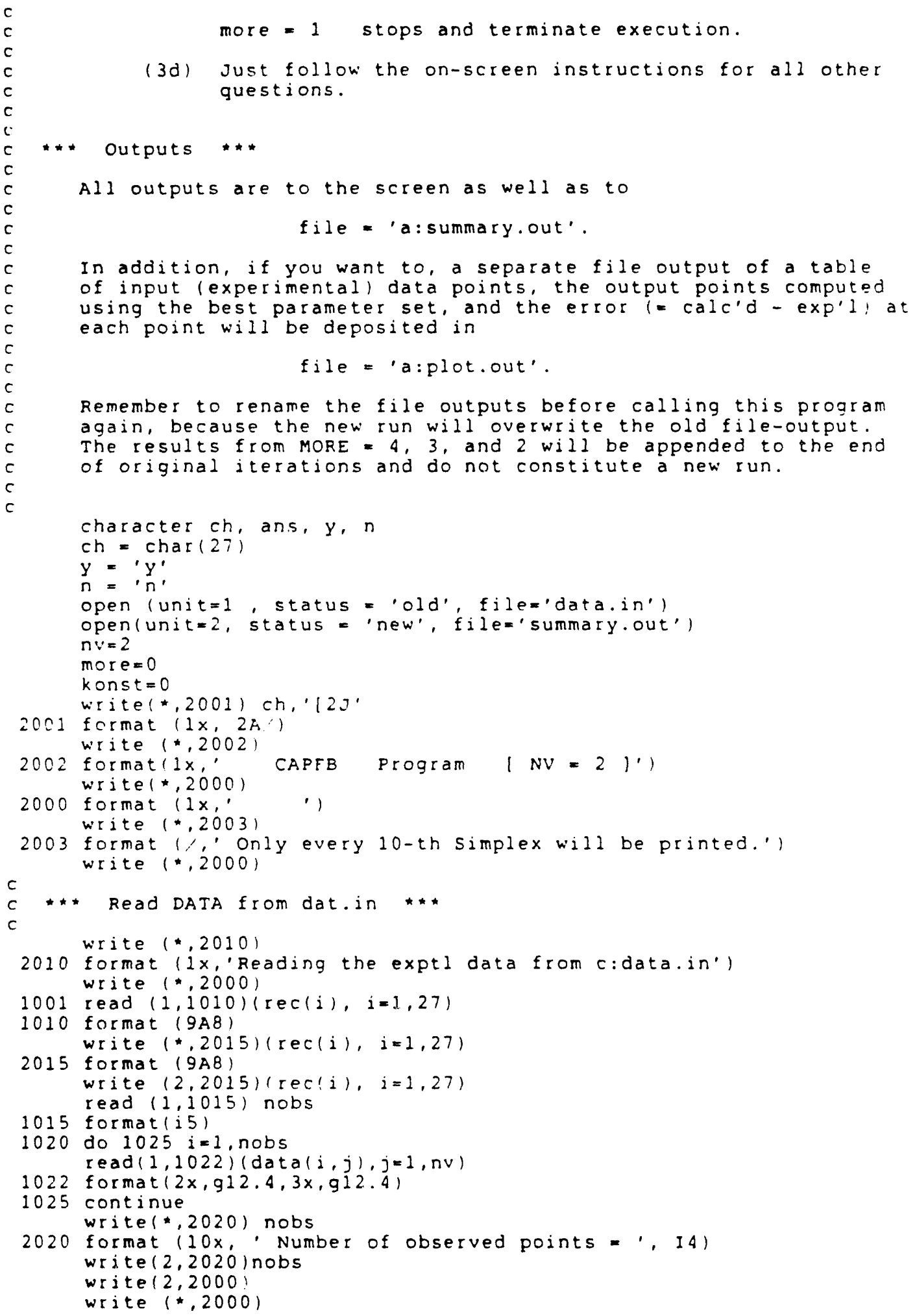


wite $(*, 2025)$

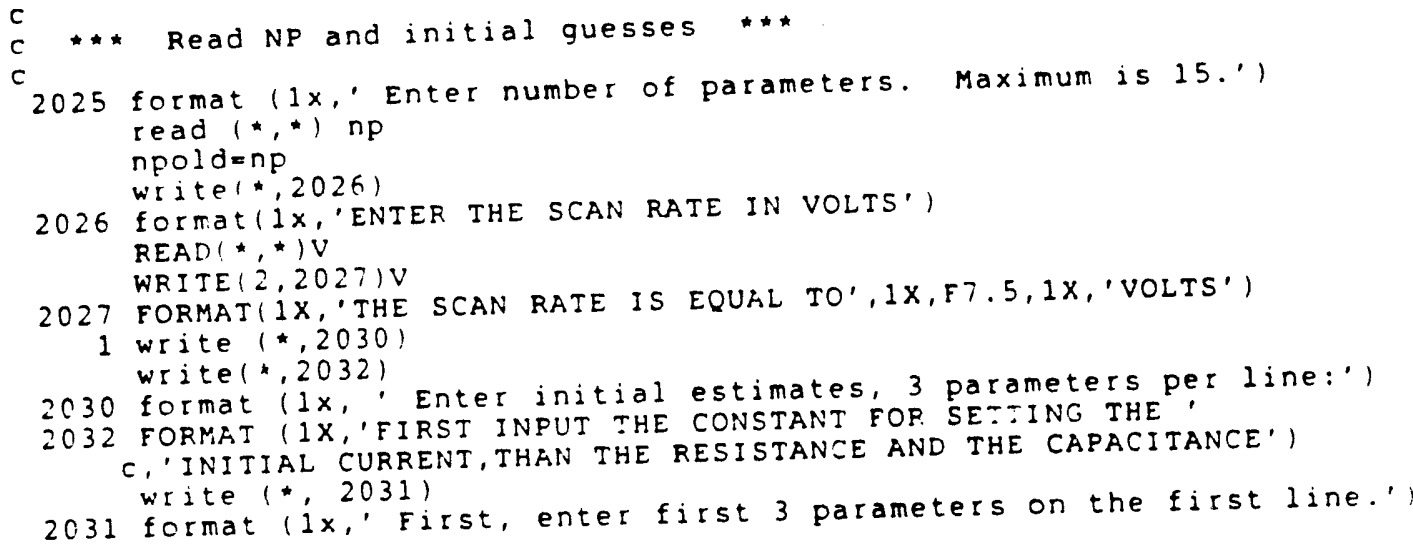




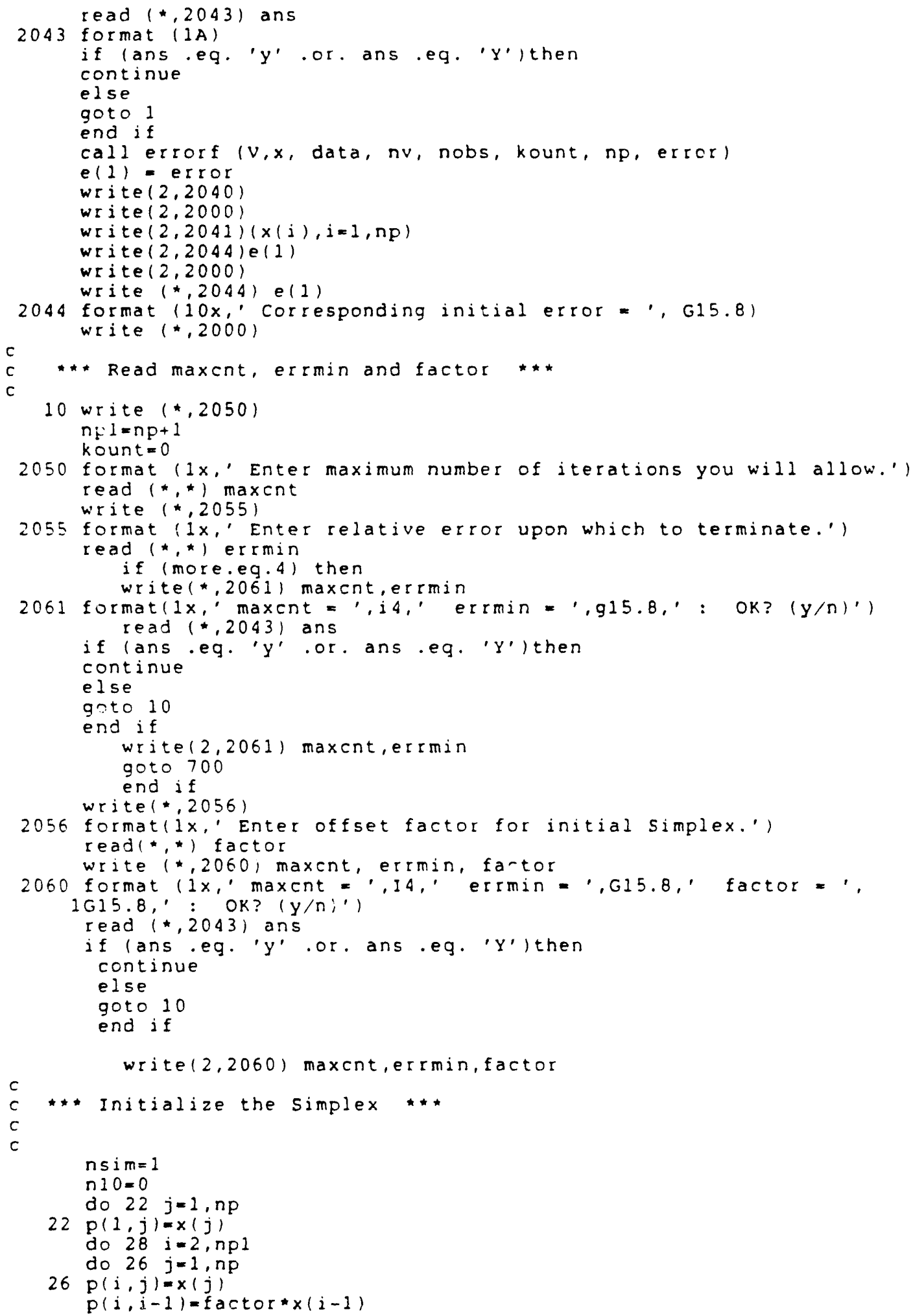




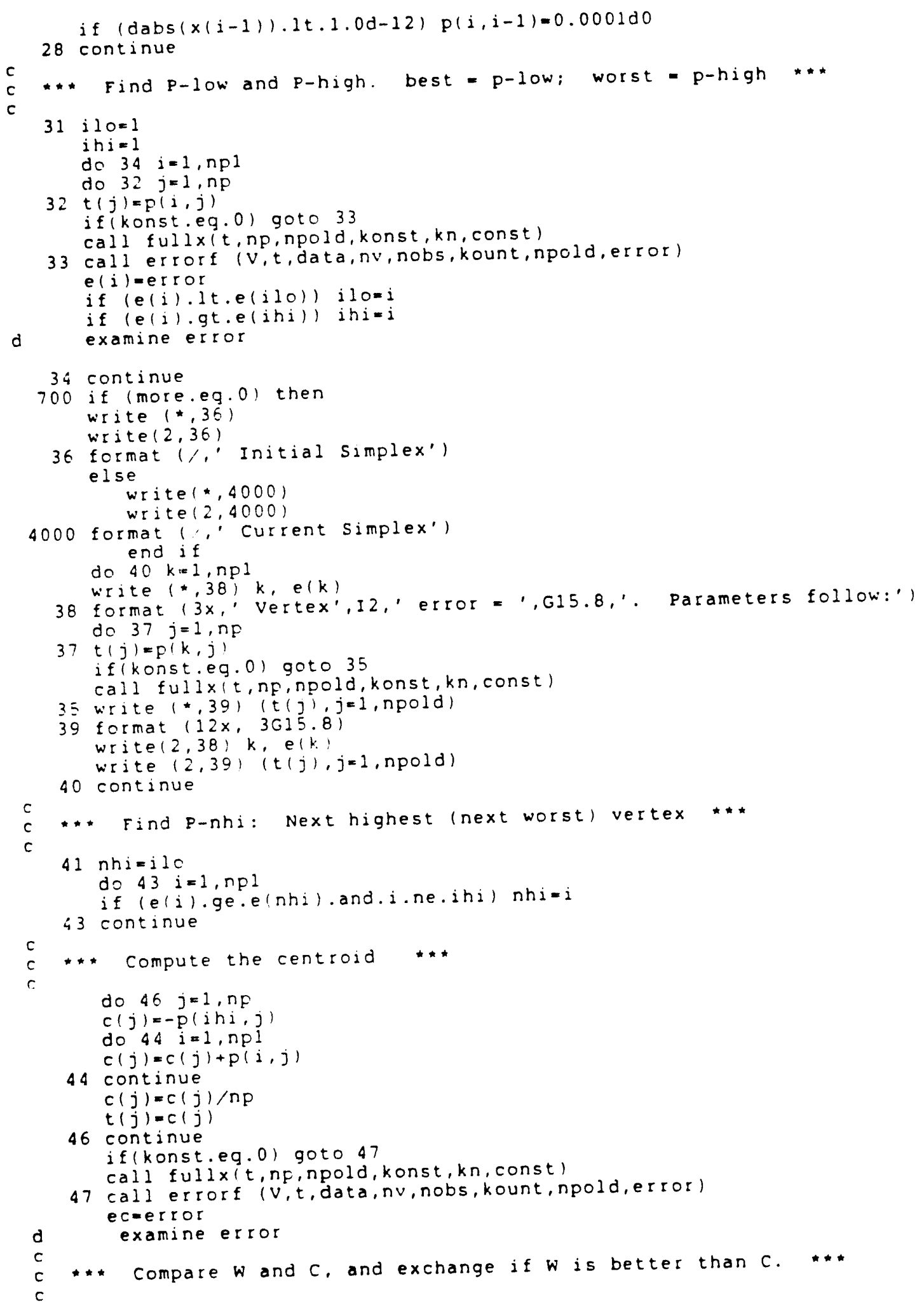




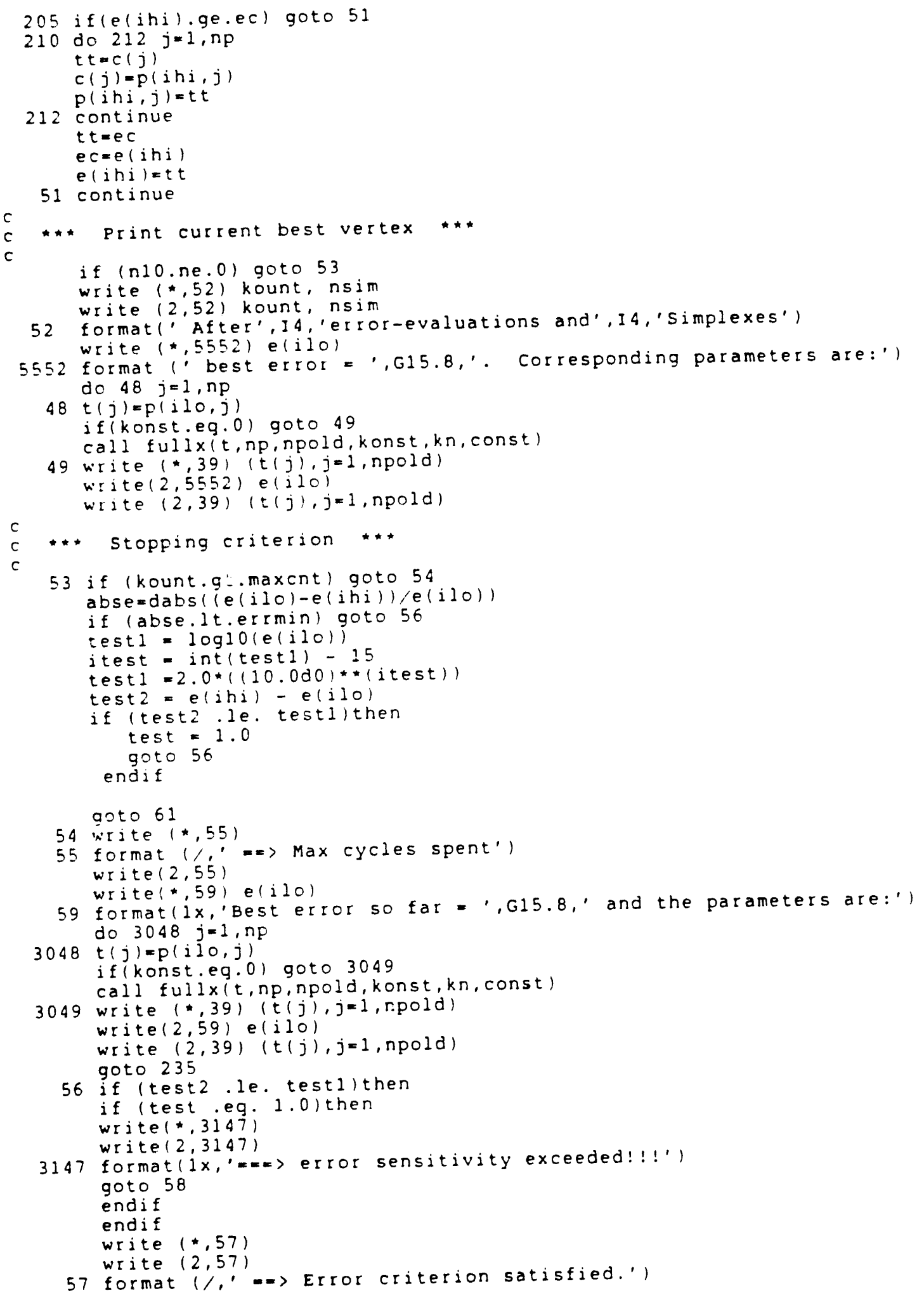




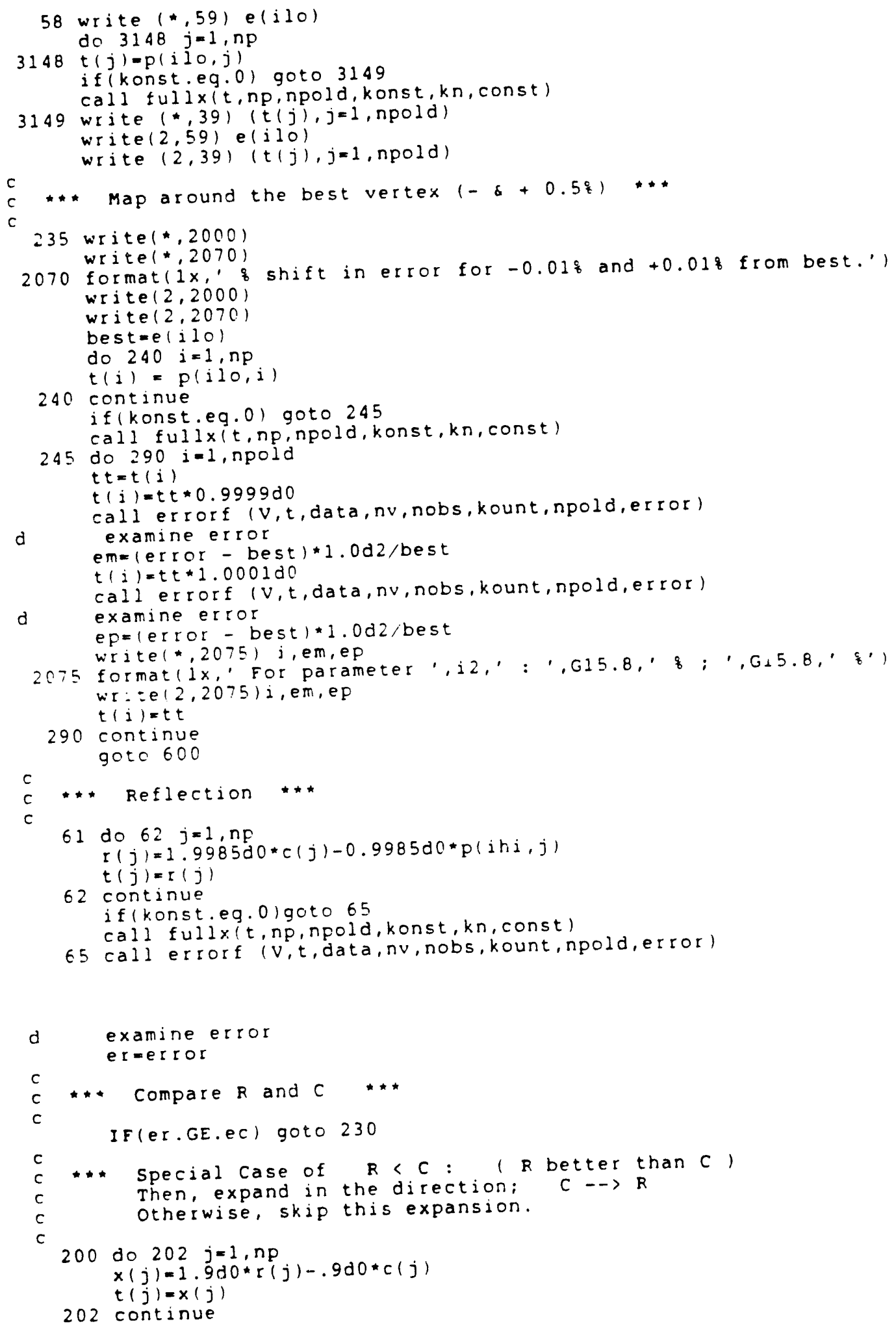




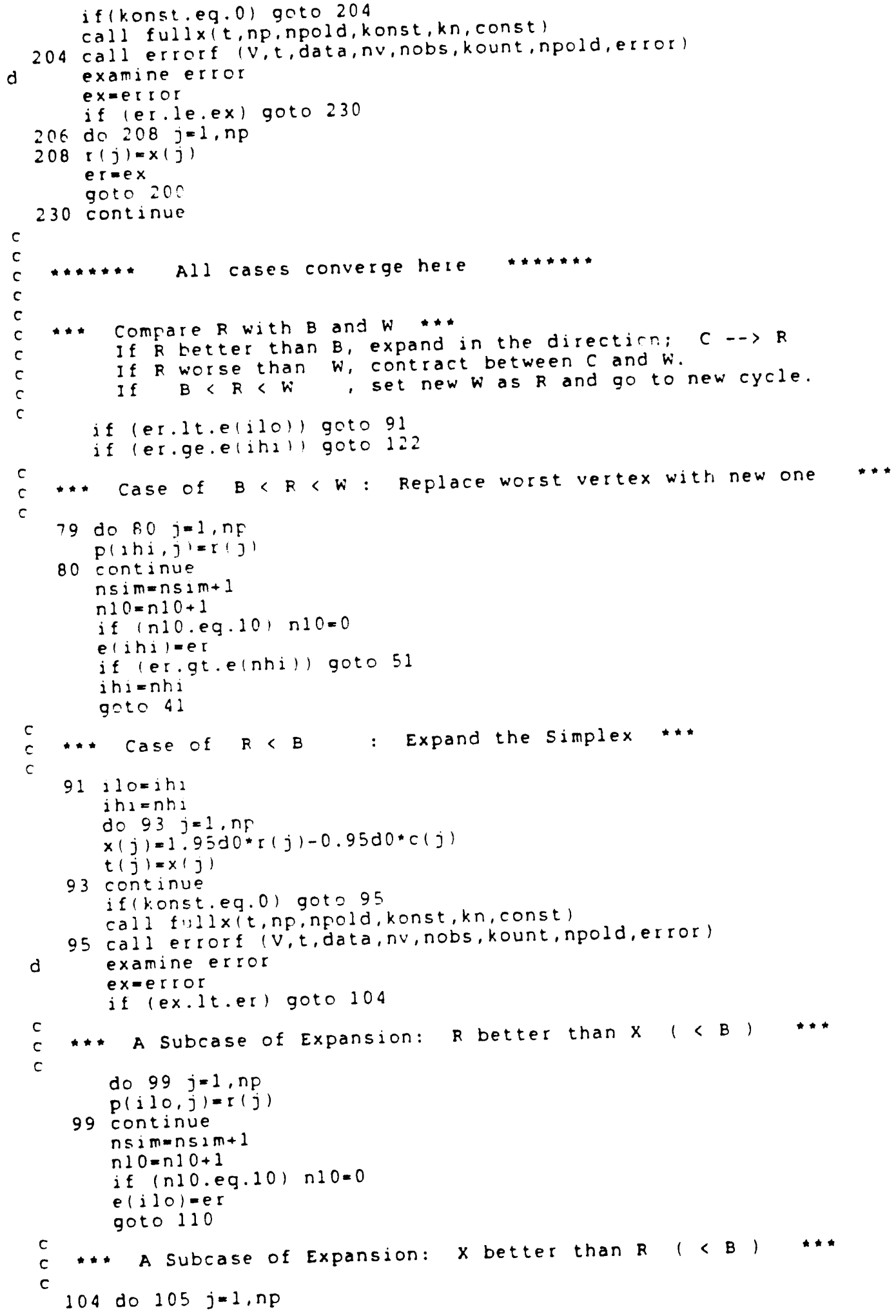




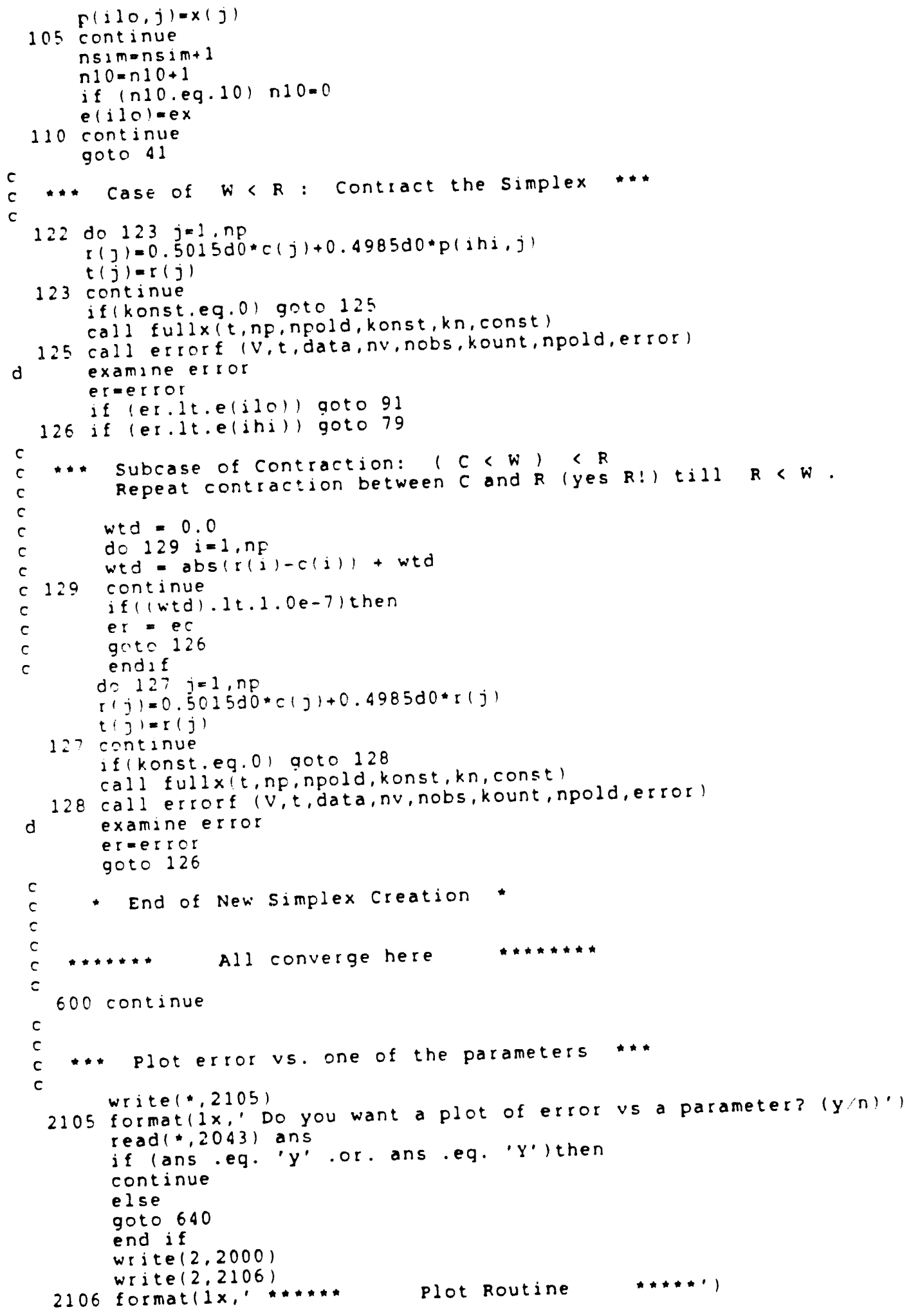




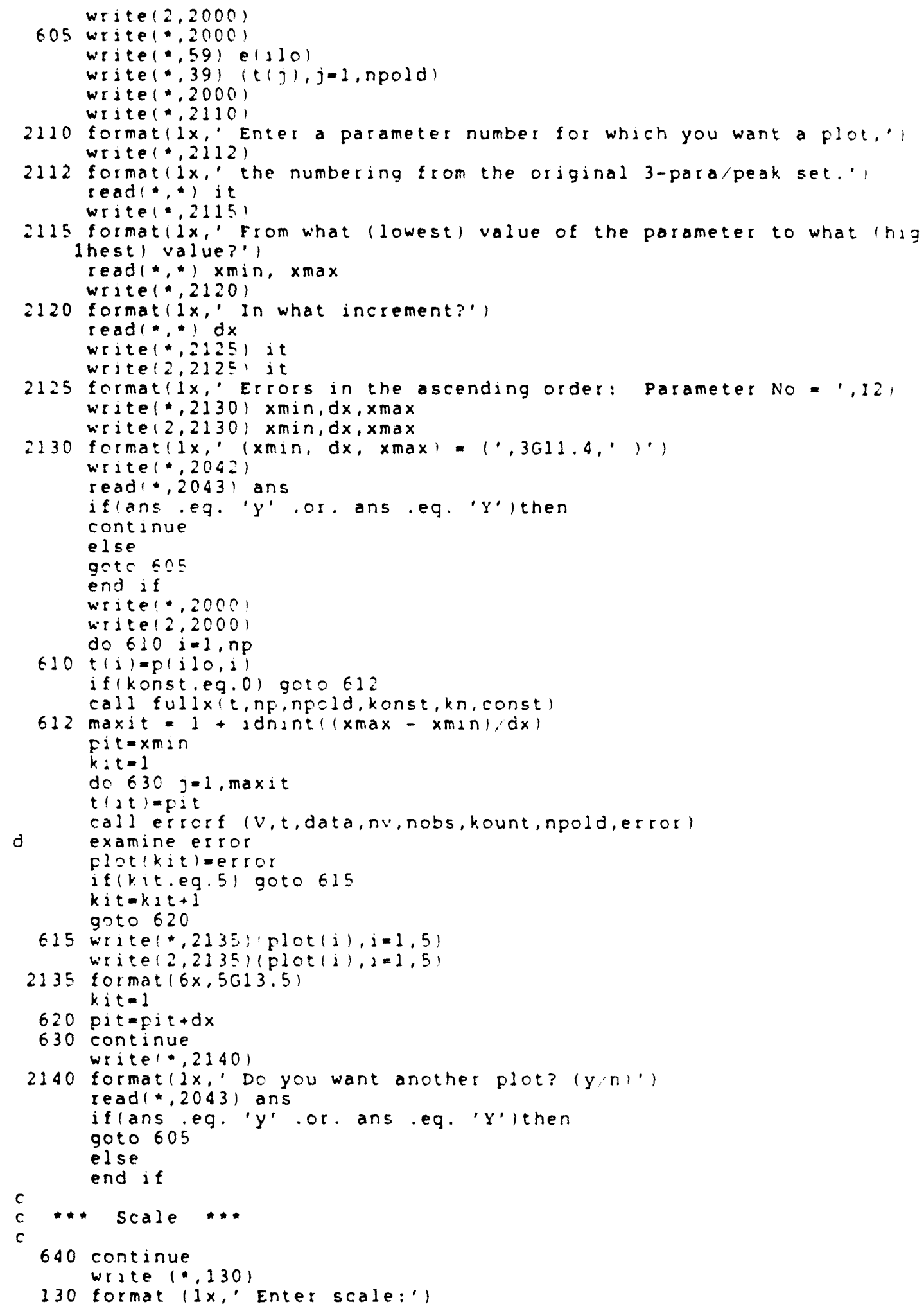




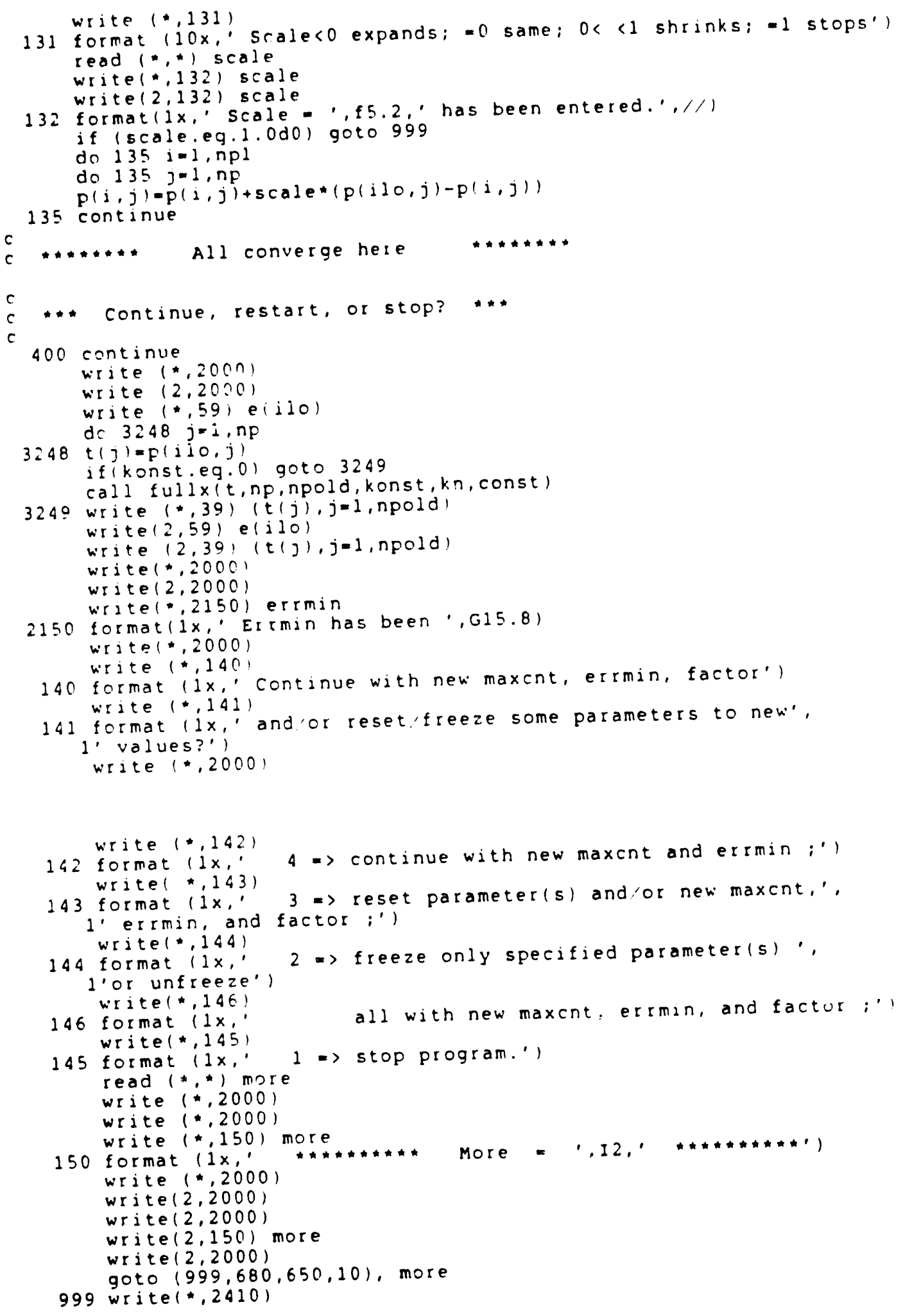




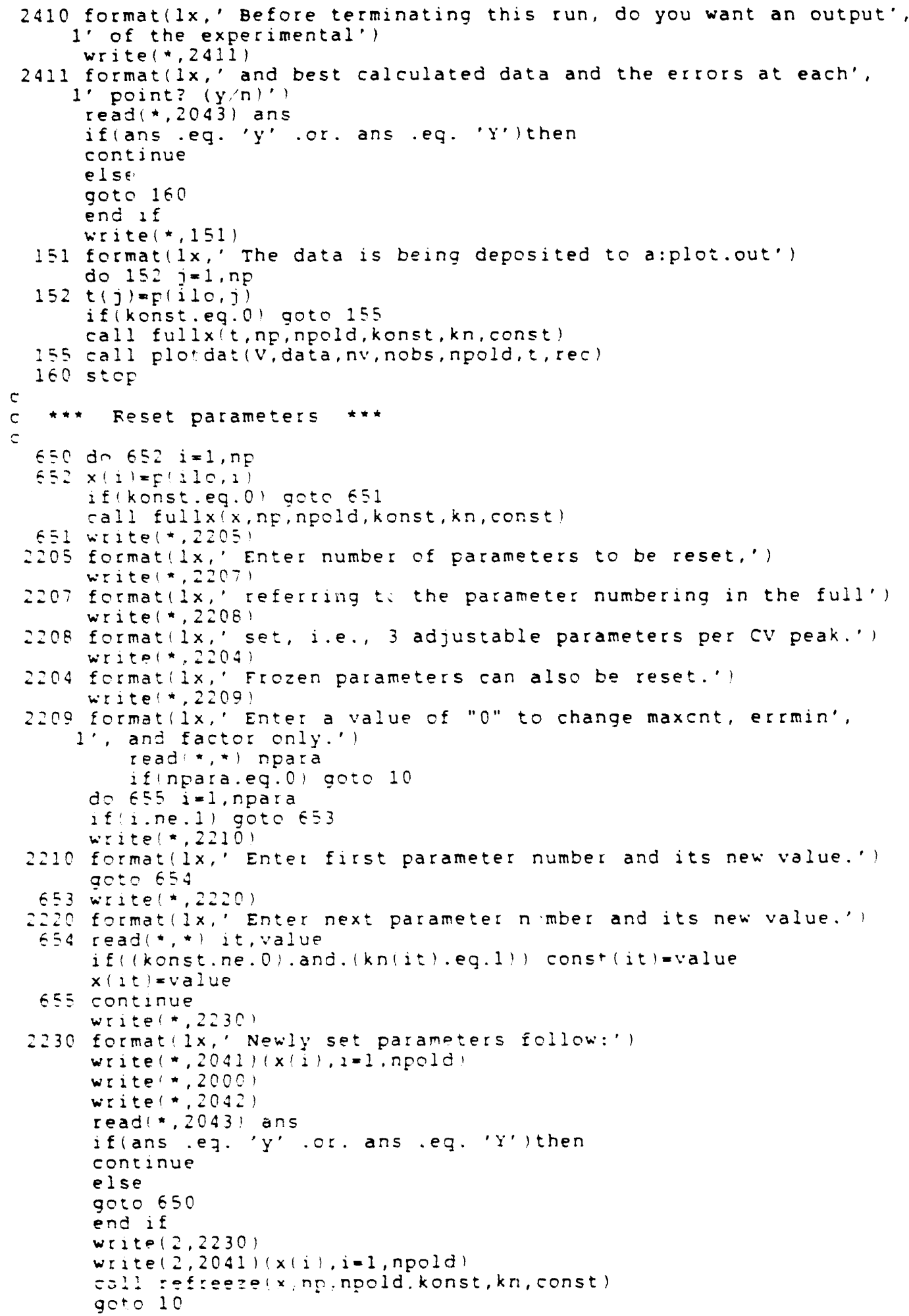




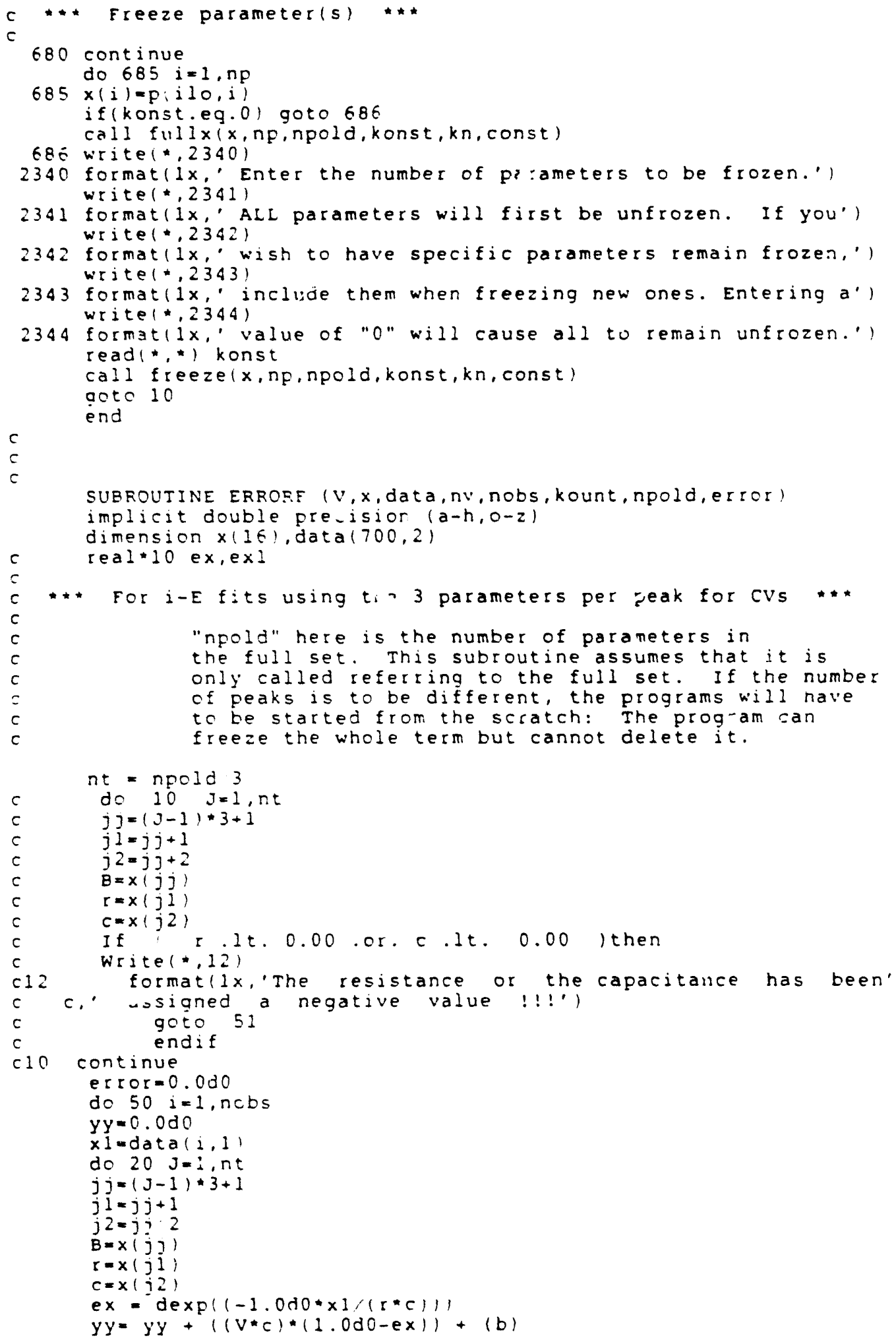




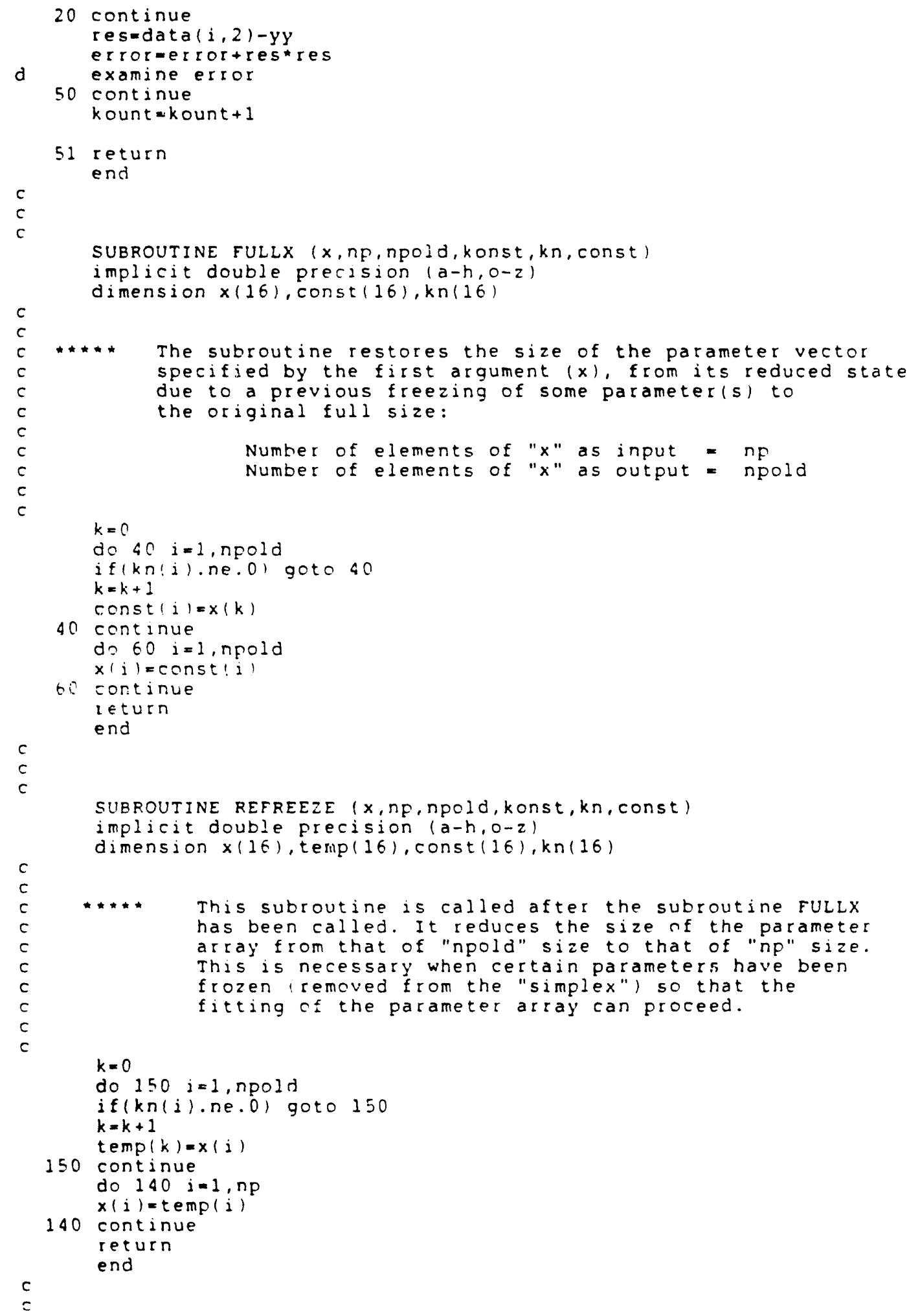




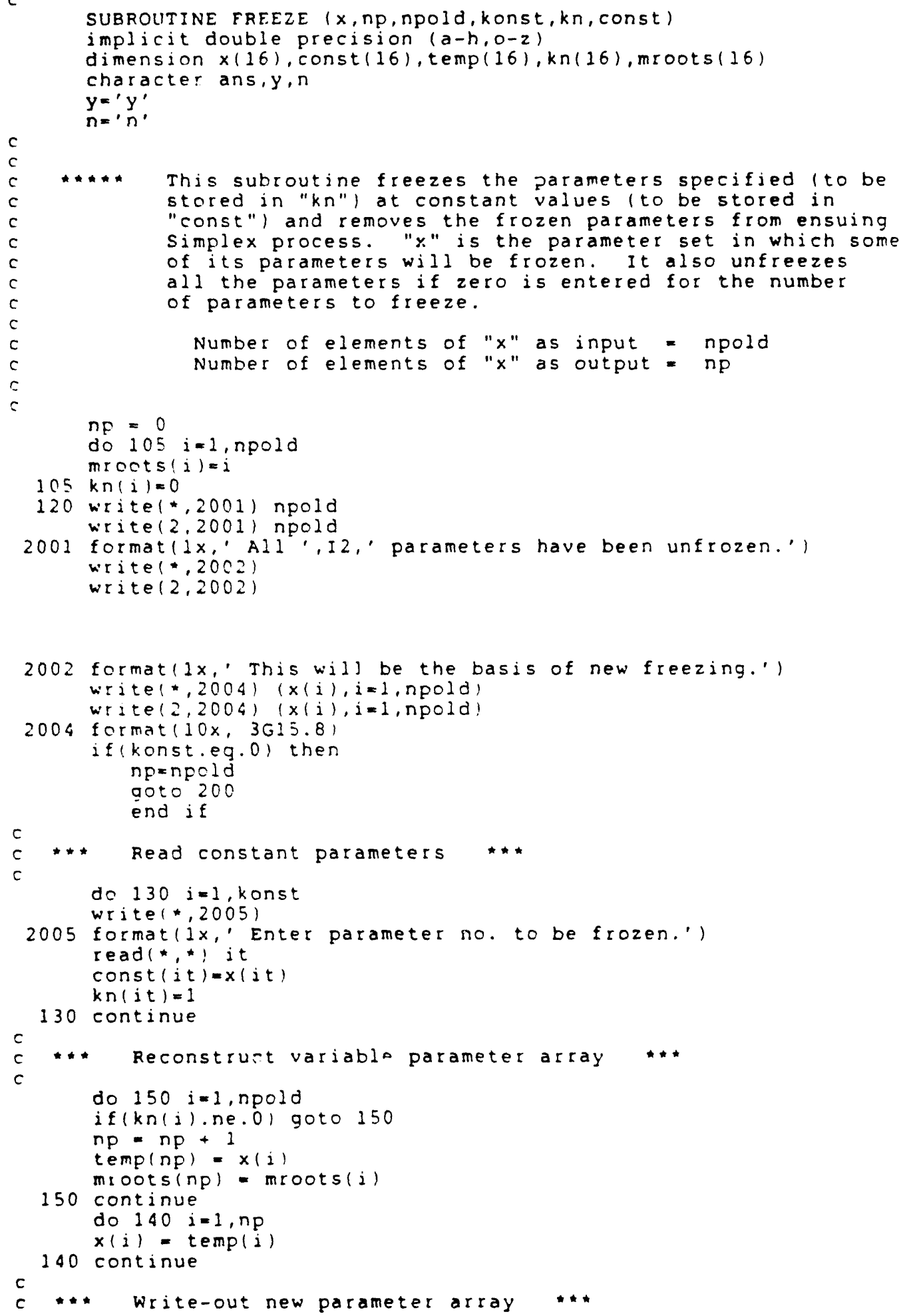




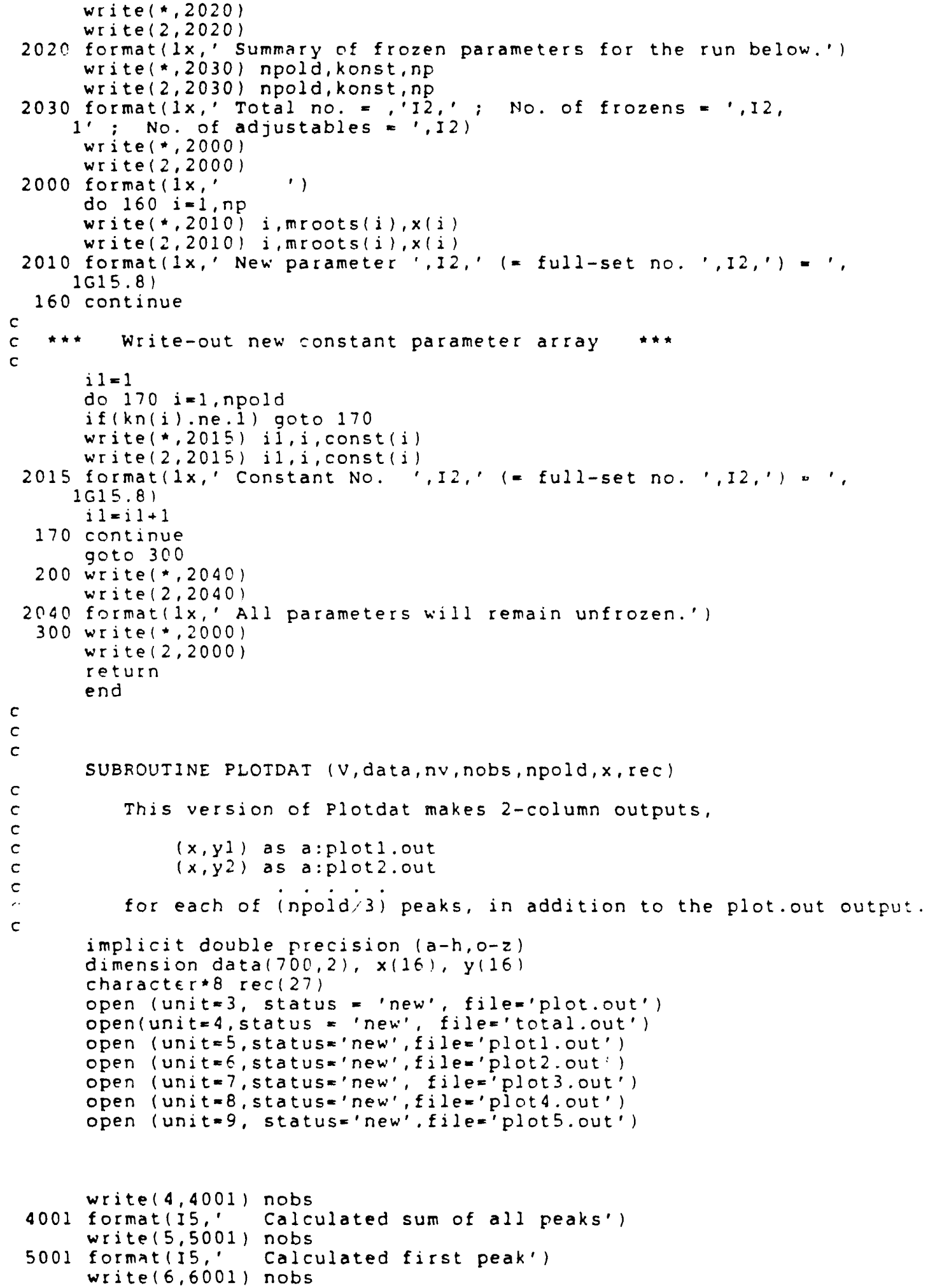




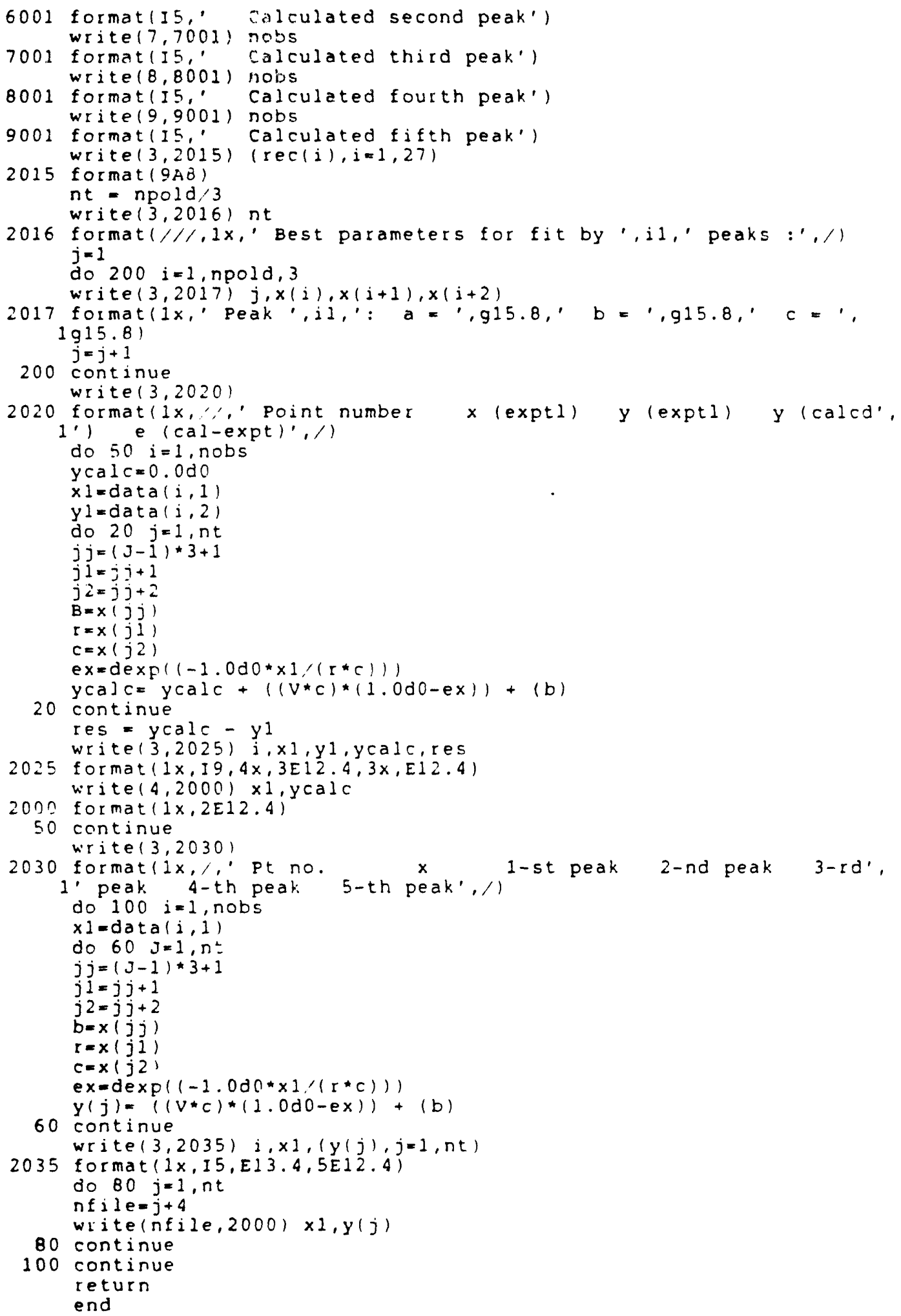




\section{LIST Of REFERENCES:}

1. Gabe, D. R. Metallurgia. 1965, 429, pp 47-52. 2. Yeager, E.; Salkind, A. J. Techniques of Electro-
chemistry; John Wiley \& Sons: New York, 1972; vol. 1 , P 295.

3. Grahame, D. C. Chem Rev. 1947, 41, pp 441-501.

4. Bockris, J. O; Yeager, E. Comprehensive Treatise of Electrochemistry; Plenum: New York, 1980; vol 1, p 293.

5. Yeager, E.; Salkind, A. J. Techniques of Electrochemistry; John Wiley \& Sons: New York, 1980; vol 4, p 211.

6. Technical Information Union Carbide Corp. (advanced ceramics); Pyrolytic Graphite.

7. Sawyer, D. T.; Roberts, J. L. Experimental Electrochemistry for Chemists; John Wiley \& Sons: New York.

8. Mann, C. K., In Electroanalytical Chemistry; Bard, A. J., Ed.; Marcel Dekker: New York, 1969;; Pp 64-65.

9. Hu, Ing-Feng. Ph.D Thesis, The Ohio State University, 1985.

10. Kuwana, T. Anal, Chem,. 1985, 57, 2759-2763.

11. Randin, J. P.; Yeager, E. J.Electroanal. Chem.. 1975 , 58, pp 313-322.

12. Randin, J. P.; Yeager, E. J. Electroanal. Chem.. 1972 , 36, pp 257-276.

13. Randin, J. P.; Yeager, E. J. Elecrochem, Soc. 1971,118 , pp 711-714.

14. Oren, Y.; Tobias, H.; Soffer, A. J.Eleztroanal Chem. 1984, P 87-99.

15. Tobias, H.; Soffer, A. J, Electroanal. Chem. 1983, 148, PP 221.232.

16. Oren, Y.; Soffer, A. J, Electroanal, Chem. 1985, 186, PP 63-77. 
17. Oren, Y.; Soffer, A. J. Electroanal. Chem.. 1986, 206, pp $101-114$.

18. Golub, D.; Soffer, A.; Oren, Y. J. Electroanal. Chem.. 1989, 260, pp 383-392.

19. Gagnon, E. G, J, Electrochem Soc, 1974, 121: no 11, PP $1444-1447$.

20. Gagnon, E. G. J. Electrochem Soc,. 1975, 122: no 4, Pp 525 .

21. Meyers, R. A.; Cowherd, W. F.; Steuernage 1, W. H. Comparison of Potential Step and Triangular Voltage Sweep Methods of Double Layer Capacitance Measurernents II. Porous Silver Electrodes.; Harry Diamond Laboratories. U.S. Army Material Command, Washington D. C. 20438

22. Gagnon, E. G. J. Electrochem. Soc.. 1974, 121, pp 512515 .

23. Delahay, P. Double Layer and Electrode Kinentics; Wiley-Interscience: New York, 1965.

24. Austin, L. G.; Gagnon, E. G.; J. Electrochem Soc.. 1973, 120: no 2, pp 251-254.

25. Bockris, J. O.; Reddy, A. K. N. Modern Electrochemistry; Plenum: New York, 1970; vols $1 \& 2$.

26. Laboratory Techniques in Electroanalytical Chemistry; Kissinger, P. T.; Heineman, W. R. Eds.; Marcel Dekker: New York, 1984.

27. Engstrom, R. C.; Strasser, V. A. Ana1, Chem. 1984, 56, pp 136-141.

28. Epstein, B. D. Carbon. 1971, 9, pp 609-615.

29. Kinoshita, K.; Bett, J. A. S. Carbon. 1973, 11, pp 403411.

30. Boehm, H. P. In Advances in Catalysis and Related Subjects; Eley, D. D.; Pines, H.; Weisz, P. B. Eds.; Academic Press: London, 1966; vol. 16, p 181.

31. Puri, B. R. In Chemistry and Physics of Carbon; Walker, P. L. Ed.; Marcel Dekker: New York, 1970; vol. 6, pp 191275 . 
32. Thomas, J. W. ; Evans, E. L.; Barber, M.; Swift, P. Trans Earaday Socfety. 1971, 67, pp 1875-1885.

33. Organic Electrochemistry; Baizer, M. M. Ed.; Marcel Dekker: New York, 1973.

34. Evans, J. F.; Kuwana, T. Ana1. Chem. 1977, 49, pp 1632 1635 .

35. Nagoaka, T.; Fukunaga, T.; Yoshino, T. J. Electroanal. Chem.. 1987, 217, pp 453-456.

36. Nagaoka, T.; Fukunaga, T.; Yoshino, T.; Watanabe, I.; Nakayama, T.; Okazaki, S. Anal. Chem. 1988, 60, Pp 27662769 .

37. Chang, J.; Large, R. F.; Popp, G. In Techniques of Chemistry: Physical Methods of Chemistry. part IIb; Weissberger, A. Ed.; Wiley-Interscience: New York, 1971; vol. 1, pp 74-75.

38. Koltoff, I. M.; Coetzee, J. F. J.Am. Chem. Soc.. 1957, 29. pp 870-874.

39. Dresselhaus, M. S. Material Sclence and Engineering. 1988, BI, pp 259-277.

40. Besenhard, J. 0.; Fritz, H. P. Z. Naturforsch. 1972, 27b, pp 1294-1298.

41. Besenhard, J. O Carbon. 1976, 14, pp 111-115.

42. Besenhard, J. O.; Mohwald, H.; Nickl, J. J. Carbon. 1980, 18, PP 399-405.

43. Simonet, J.; Lund, H. J.Electroanal. Chem., 1977, 75, pp $719-730$.

44. Bernard, G.; Simonet, J. J.Electroanal. Chem.. 1980, 112, pp 117-125.

45. Gewirth, A. A.; Bard, A. J. J. Physical Chem.. 1988, 92 , PP 5563-5566.

46. Besenhard, J. O.; Fritz, H. P. Angew Chem, Int Ed, 1983, 22, pP $950-975$.

47. Bertholot, J.; Simonet, J. Electrochim. Acta. 1984, Vol. 29: No 4, pp 1181-1186. 
48. Fischer, J. E.; Thompson, T. E. Phystes Today. 1978, 31. pp $36-45$.

49. Krebs, L. C.; Ishida, T., The State University of New York at Stony Brook, personal communication.

50. Engstrom, R. C. Anal Chem, 1982, 54, pp 2310-2314.

51. Miller, C. W.; Karweik, D. H. ; Kuwana, T. Anal. Chem,. 1981, 53, pp 2319-2323.

52. Poon, M.; McCreery, R. L.; Engstrom, R. Anal Chem. 1988, 60, pp 1725-1730.

53. Adams, R. N. Electrochemistry at Solid Electrodes; Marcel Dekker: New York, 1965; Pp 43-61.

54. Sharp, M. Electrochim. Acta. 1983, Vo1, 28, No. 3, PP $301-308$.

55. Bond, A. M.; Henderson, T. L. E.; Mann, D. R.; Mann, T. F. ; Thormann, W.; Zoski, C. G. Anal. Chem.. 1988, 60, pp $1878-1882$.

56. Coury, L. A.; Heineman, W. R. J, Electroanal Chem. 1988, 256, Pp 327-341.

57. Coury, L. A.; Birch, E. M.; Heineman, W. R. Anal. Chem. 1988,60 , pp 553-560.

58. Daschbach, J.; Blackwood, D.; Pons, J. W. ; Pons, S. J. Electroanal. Chem, 1987, 237, pp 269-273.

59. Gritzner, G.; Kuta, J. Pure Appl. Chem.. 1984, 56, pp $461-466$.

60. Fann, Y-C.; Hao, H-S; Kapoor, R. C. J. of Chin. Chem. Soc. 1989, 36, Pp 21-24. 

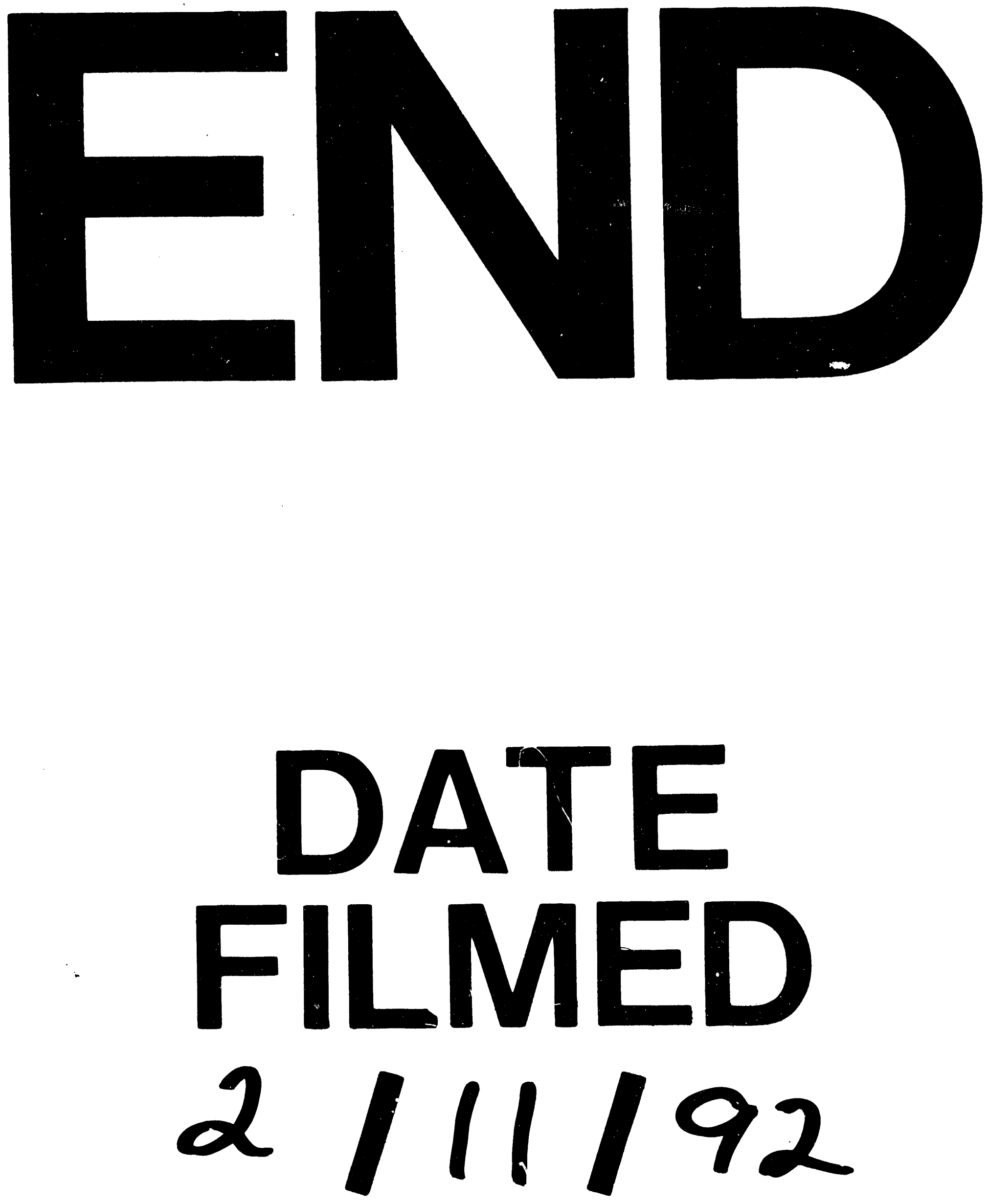

1 
1

1 